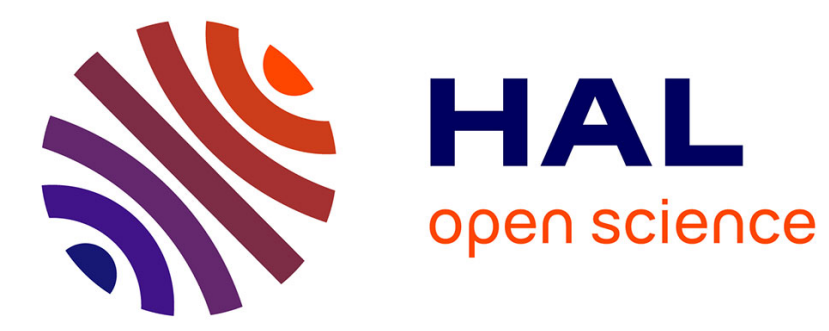

\title{
Advances in the synthesis of 1,2-dioxolanes and 1,2-dioxanes
}

Laurent Ferrié

\section{To cite this version:}

Laurent Ferrié. Advances in the synthesis of 1,2-dioxolanes and 1,2-dioxanes. Advances in Heterocyclic Chemistry, 135, pp.57-146, 2021, 10.1016/bs.aihch.2021.03.001 . hal-03320615

\section{HAL Id: hal-03320615 https://hal.science/hal-03320615}

Submitted on 15 Oct 2021

HAL is a multi-disciplinary open access archive for the deposit and dissemination of scientific research documents, whether they are published or not. The documents may come from teaching and research institutions in France or abroad, or from public or private research centers.
L'archive ouverte pluridisciplinaire HAL, est destinée au dépôt et à la diffusion de documents scientifiques de niveau recherche, publiés ou non, émanant des établissements d'enseignement et de recherche français ou étrangers, des laboratoires publics ou privés. 


\section{Recent Advances in the Chemistry of 1,2-Dioxolanes and 1,2-Dioxanes}

\section{Table of content}

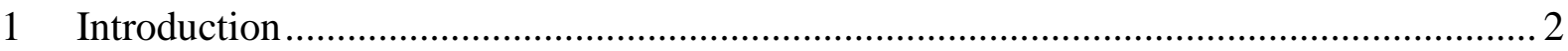

2 Natural products with 1,2-dioxolanes and 1,2-dioxanes scaffolds ................................. 3

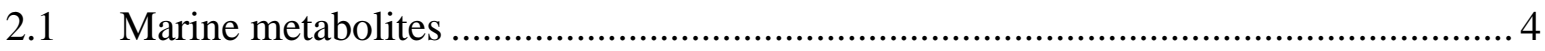

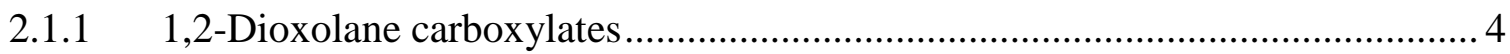

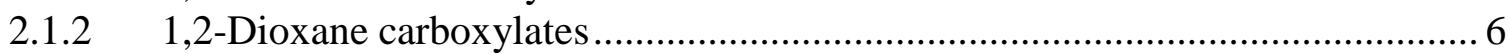

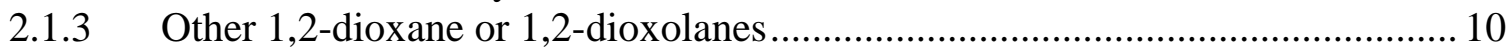

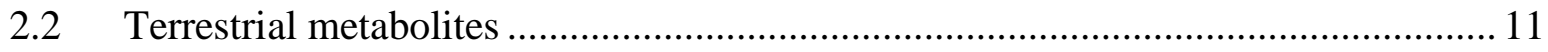

2.2.1 1,2-Dioxolanes and 1,2-dioxanes isolated from plants ................................... 11

2.2.2 1,2-Dioxolanes and 1,2-dioxanes isolated from other kingdoms....................... 14

3 Methods to synthesize 1,2-dioxolanes, 1,2-dioxanes and 1,2-dioxenes. ......................... 16

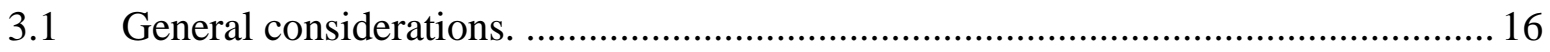

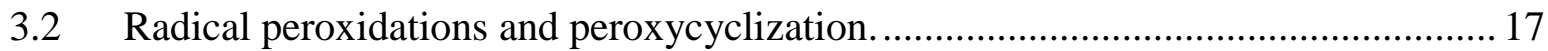

3.2.1 Radical addition of triplet oxygen and direct formation of an endoperoxide. ... 17

3.2.1.1 Cycloaddition of triplet oxygen onto diradical species. ................................. 17

3.2.1.2 Formal cycloaddition of triplet oxygen onto radical cations .......................... 18

3.2.1.3 Formal cycloaddition of triplet oxygen to cycloalkoxy radicals .................... 24

3.2.1.4 Formal $[2+2+2]$ cycloaddition of triplet oxygen to enoxy radicals and olefins 27

3.2.1.5 Isayama-Mukaiyama cobalt catalyzed hydroperoxy-silylation .......................30

3.2.1.6 Thiyl and selenyl radical-mediated domino reactions .................................... 35

3.2.1.7 Organozinc oxidation through an electrophilic activation of a cyclopropane 40

3.2.2 Radical Peroxycyclization of Hydroperoxides............................................... 41

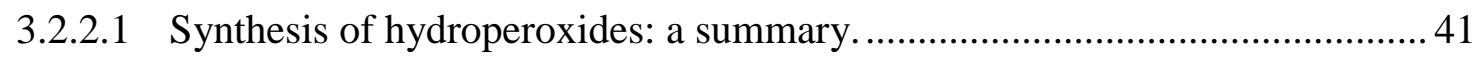

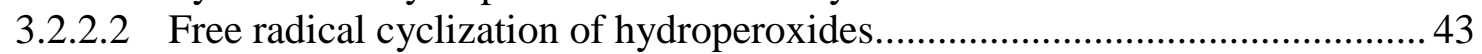

3.3 Cyclization through nucleophilic addition or substitution ...................................... 44

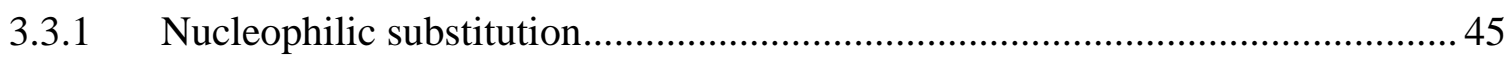

3.3.1.1 Nucleophilic substitution $\left(\mathrm{SN}_{2}\right)$ on halides or sulfonates ...............................45

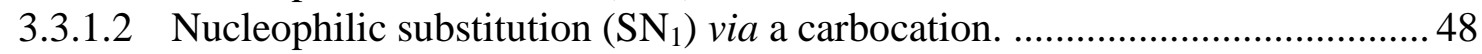

3.3.1.3 Nucleophilic substitution on strained heterocycles ....................................... 49

3.3.2 Addition to carbon-carbon double bonds and related functions .........................54

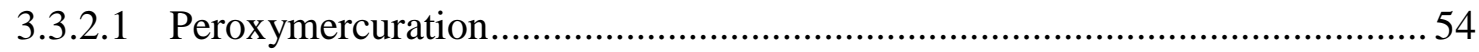

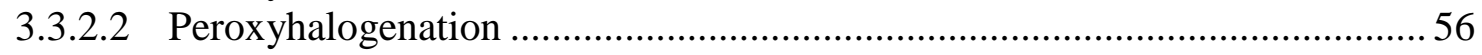

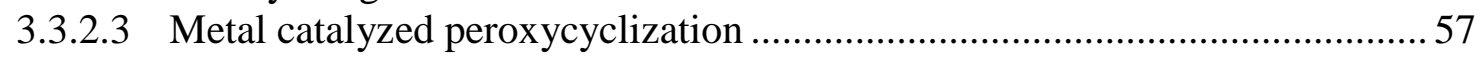


3.3.2.4 Conjugated addition.

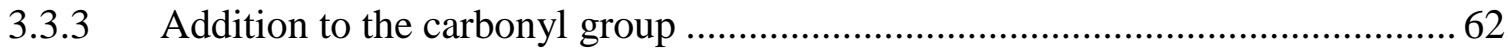

3.4 Peroxycylization involving Electrophilic Singlet Oxygen .................................... 66

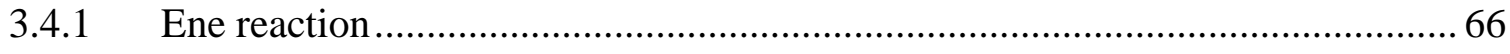

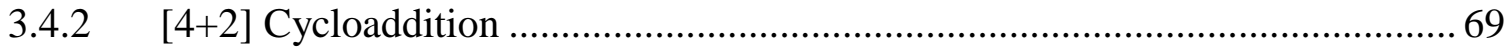

3.5 Peroxycylization and Functionalization of Endoperoxyketals through the Generation

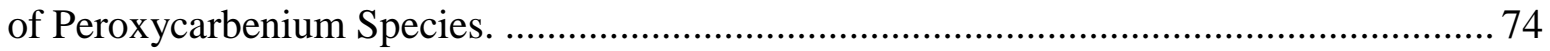

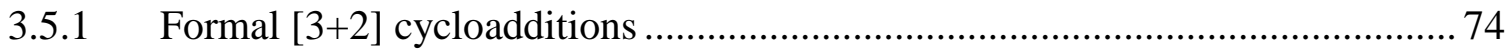

3.5.2 Functionalization of Endoperoxyketals and acetals ...................................... 79

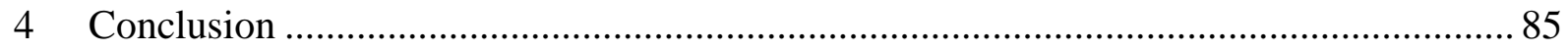

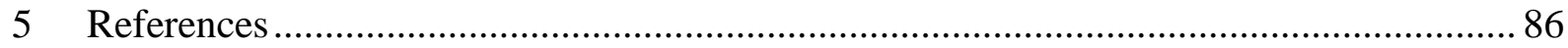

\section{Introduction}

1,2-Dioxolanes and 1,2-dioxanes are particular examples of endoperoxides, which can be found in many natural substances, but have also many perspectives in medicinal chemistry. Indeed, endoperoxides are very famous class of compounds to possess anti-malaric and more generally anti parasitic properties. 1,2,4-Trioxanes or 1,2,4-trioxolanes are for example the main pharmacophore of anti-malarial natural artemisinin and synthetic arterolane (Figure 1).

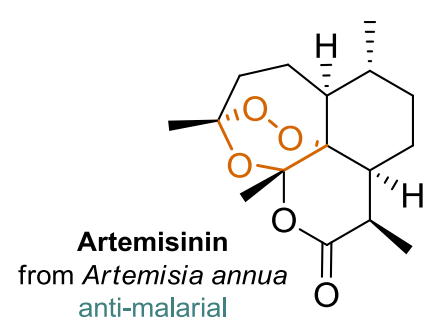

1,2,4-trioxanes
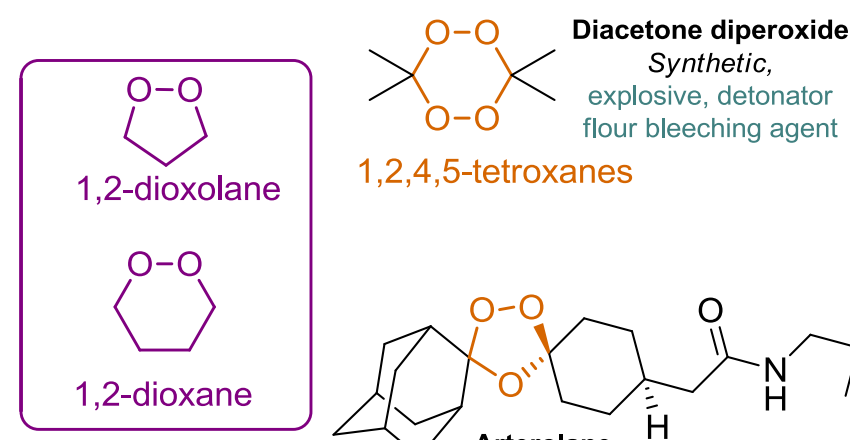

1,2,4,5-tetroxanes

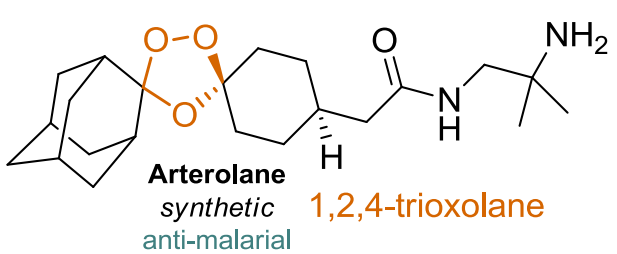

Figure 1. Structures of 1,2-dioxolane and 1,2-dioxane. Structures of artemisinin, arterolane and diacetone piperoxide, exhibiting structures of 1,2,4-trioxane, 1,2,4-trioxolane and 1,2,4,5tetroxane.

However, 1,2-dioxolanes and 1,2-dioxanes exhibit also different biological properties, including anti-malarial activities, and by consequence these structures attracted several synthetic chemists for many years in order to develop efficacious methods to prepare these oxygenated heterocycles. Most chemists fear organic 
peroxides owing to their reactive and explosive character, such as with diacetone diperoxide (Figure 1). However, 1,2-dioxolanes and 1,2-dioxanes are in general stable compounds under normal conditions due a lower proportion of oxygen atoms involved in peroxide bonds compared to other atoms and a stabilization of the peroxide bond within a favorable 5- or 6-membered ring. Thus, they can generally be easily purified, for example, by silica gel chromatography. Since structures and methods to obtain 1,2-dioxanes and 1,2-dioxolanes are somewhat similar, and given that some families of natural product contain both heterocycles, it seems legitimate to treat both in a single chapter.

Cyclic peroxides were reviewed more than 50 years ago in Advances in Heterocyclic Chemistry ${ }^{1}$ therefore, an updated version reviewing most recent works and methods is today required. More recent reviews ${ }^{2,3,4}$ and also book chapters5,6 depicted the subject in a more general manner. This chapter encompasses some work presented in these review articles/books, in order to give a general overview of the most successful methods for synthesizing 1,2-dioxolanes and 1,2-dioxanes, but also will focus on the most recent strategies published these 15 last years in the field. A presentation of the natural products containing these structures is a good starting point to understand the importance of developing new methods for the synthesis of 1,2-dioxanes and 1,2-dioxolanes. The existing techniques for preparing these particular heterocycles will be discussed later through the presentation of free radical chain reactions involving oxygen triplet, nucleophilic additions with hydroperoxides, ene reactions or [4+2] cycloadditions involving electrophilic oxygen singlet, [3+2] formal cycloaddition and functionalization of endoperoxyketals involving peroxycarbenium ion species.

\section{Natural products with 1,2-dioxolanes and 1,2-dioxanes scaffolds}

Although organic peroxides have a reputation of being reactive and unstable species, it exists a large number of natural products containing 1,2-dioxolane and 1,2dioxane rings. Their different structures are exhaustively reported in some reviews, $, 7,8,9$ so this part could be consider as a summary of all reported structures. The different natural substances are going to be presented following their origin and their structural family to which they belong, focusing more on original and important structures. 


\subsection{Marine metabolites}

Marine resources are major sources of endoperoxides, and sponges are in particular the most important pool for this class of compounds. Some cytotoxic or antifungal activities are often reported for these compounds. Thus, primary biological task of these compounds is probably to play a role of chemical defender against certain predators.

\subsubsection{1,2-Dioxolane carboxylates}

1,2-Dioxolanes are in proportion a minor structure among the peroxy natural products, however sponges of Plakortis and Plakinastrella species are providing a large number of these scaffolds. Plakinic acids A, ${ }^{10} \mathrm{C}, \mathrm{D},{ }^{11} \mathrm{E},{ }^{12} \mathrm{~F},{ }^{13,14} \mathrm{G}, \mathrm{H},{ }^{15} \mathrm{~J},{ }^{16} \mathrm{~L},{ }^{17}$ $\mathrm{N}^{18}$ exhibit a fatty acid structure bearing a dioxolane between position 3 and 5 . Two methyl substituents are also present at these positions. Their absolute configuration is generally inconsistent and depends on the sponge species studied, leading to one or the other enantiomer. Epimers at $\mathrm{C}_{5}$, with trans configuration at position 3 and 5 , are called epiplakinic acids, which make it possible to distinguish between cis and trans diastereomers. Only the isolation work of epiplakinic acids $\mathrm{G}$ and $\mathrm{H}$ was ambiguous since the authors misused the term "epi", driving to some confusion.15 Indeed, epiplakinic acid $\mathrm{H}$ should have been called plakinic acid $\mathrm{G}$ to follow the general rule. The absolute stereochemistry was generally determined either by total synthesis such as with plakinic acid A, 19 Mosher ester analysis of the reduced 1,2-dioxolane ring ${ }^{20}$ or by NMR analysis of the diol with some chiral lanthanum shifting agent, ${ }^{21}$ but recently, circular dichroism also proved to be a reliable method. ${ }^{16}$ However attention must be taken when using chiral lanthanum shifting agent, because epiplakinic acid $\mathrm{F}$ seemed to have been incorrectly assigned, as demonstrated by its total synthesis, where the optical sign matched with the opposite configuration. ${ }^{22}$ Indeed optical rotation is strongly driven by the stereochemistry at $\mathrm{C}_{3}$ and $\mathrm{C}_{5}$ in this products family, cis and trans stereoisomers giving an opposite sign and the fatty chain exerting a lesser influence on it (Figure 2). 

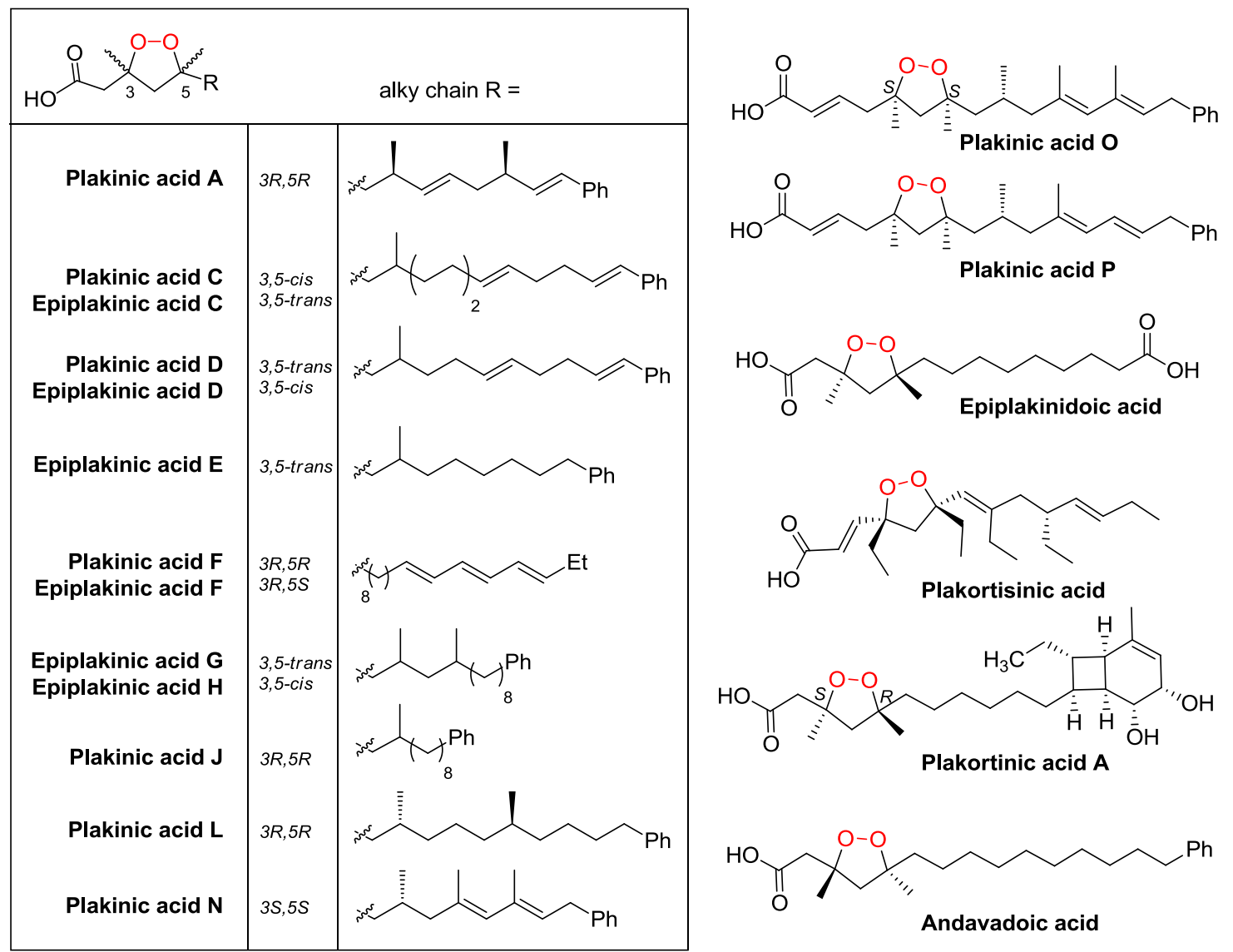

Figure 2. Major known 1,2-dioxolanes metabolites isolated from marine sponges Plakortis or Plakinastrella sp.

The main difference between all plakinic acids relies on the side chain, which provides different chain lengths, unsaturations, and ramifications. Plakinic acids $\mathrm{O}$ and $\mathrm{P}$ show a more uncommon 1,2-dioxolane placed at position 5 and 7 with a conjugated acid. ${ }^{18}$ Some other related compounds were isolated such as epiplakinidoic acid with a carboxylic acid function at the end of the fatty chain. ${ }^{14}$ or plakortinic acid A, which exhibits a beautiful bicyclo[4.2.0] octene framework. ${ }^{23}$ The structure of plakortisinic acid shows ethyl substitution on both 1,2-dioxolane ring and side chain, and a 4,6-location for the dioxolane, which is quite unusual compared to the plakinic acids series. 24 Absolute configuration was deduced from optical rotation value by comparison with a calculated one. Andavadoic acid belongs to plakinic acids since it was isolated from Plakortis aff simplex and would have deserved to be differently named. ${ }^{25}$ Absolute configuration was not reported in the original article but a total synthesis stated later its absolute configuration (Figure 2). ${ }^{26}$

A bio-guided isolation strategy was mostly at the origin of the discovery of these natural products. Therefore, some moderate antifungal (plakinic acid A, F, J, L) and moderate cytotoxic activity at the micromolar to sub-micromolar range (plakinic 
acids C, D, E, F, G, H, epiplakinoic acid, plakortinic acid, plakortisinic acid, andavadoic acid) were observed. Anti-HIV, -parasitic, or -bacterial bioassays were performed for some compounds but their activity in these fields was relatively low (Figure 2).

\subsubsection{1,2-Dioxane carboxylates}

1,2-Dioxane ring is more representative of the major part of endoperoxides isolated from marine species. The amount of members of this class being quite large, they cannot be all presented in this book chapter. Nonetheless, the major compounds will be shown with some selected examples. Indeed, such as in the plakinic acids family, only small differences exists between some members of a same family.

Most of plakinic acids, isolated from Plakortis or Plakinestrella sp., exhibit a 1,2-dioxolane ring (paragraph 2.11), but some members show also a 1,2-dioxane entity. Early isolated plakinic acids B, ${ }^{10}$ and more recent plakinic acids $\mathrm{I}^{16} \mathrm{~K}^{17}$ or $\mathrm{M}^{18}$ contain this scaffold. Similarly to plakinic acids containing a 1,2-dioxolane ring, these natural products exhibit interesting antifungal activities (Figure 3).

Plakortides, such as plakortisinic acid for 1,2-dioxolanes, were isolated from different Plakortis species and exhibit some ethyl substituents in place of classical methyl ones at positions 3 and 4 of the 1,2-dioxane ring as well as on the fatty chain. A selected sample of this large class of compounds are shown herein with plakortides $\mathrm{K}$ and $\mathrm{Z}$. This latter one is the simplest compound of the family, therefore it is a good platform for further synthetic studies towards total synthesis of such compounds. This class of natural products exhibits generally some moderate to potent antifungal and/or cytotoxic activities, however an interesting antimalarial activity was also demonstrated for some members (Figure 3 ). ${ }^{27}$

Plakortolides are a distinct class of 1,2-dioxane type natural products bearing a carboxylate function, but their originality relies on their bicyclic structure. They were also isolated from different sponge species of Plakinistrella or Plakortis genera. The stereochemistry of the stereogenic centers can be very different, depending the isolated products, such as plakortolide $\mathrm{D}^{12}$ or $\mathrm{U}, 28$ which generally lead to different alphabetical denominations. A rationalization of this stereochemistry was particularly studied, involving a questioning about the biosynthesis pathway, where a cyclization of a hydroperoxide is more likely than a [4+2] cycloaddition with ${ }^{1} \mathrm{O}_{2}$, which would 
lead to a more defined stereochemistry, because of the concerted process (see paragraph 3.4.2). ${ }^{29}$ An interesting example of a plakortide-like product is plakortoperoxide $\mathrm{C}$, which shows also a 1,2-dioxene scaffold on the side chain. This 1,2-dioxene comes certainly from a [4+2] cycloaddition between ${ }^{1} \mathrm{O}_{2}$ and a diene. These compounds exhibited also moderate to good cytotoxic activities, as well as antifungal properties. Some activity against Toxoplasma gondii parasite was also reported for some derivatives (Figure 3).30

Stolonoxides are an interesting class of molecules bearing an adjacent tetrahydrofuran ring towards the 1,2-dioxolane ring. These compounds were isolated from tunicate Stolonica socialis, and not from a sponge, contrary to most of marine 1,2-dioxane compounds. Such as plakinic acids or plakortides, this class of chemicals contains a carboxylic function with a fatty chain. However, there is no methyl or ethyl substitution on the 1,2-dioxane ring as well as on the side chain. Differences between stolonoxides A to D depend on the stereochemistry on the THF ring and the configuration of the unsaturation at $\mathrm{C} 19-\mathrm{C} 20.31,32$ Stolonoxide $\mathrm{E}$ and $\mathrm{F}$ bear an unprecedented unsaturated cyclooctane ring. 33 Stolonoxides exhibits some potent cytotoxic activities below the micromolar range against some several cell lines (Figure $3)$.

Another important class of 1,2-dioxanes isolated from marine species is the norterpene cyclic peroxides. These compounds are generally isolated from different sponge species of Prianos, Sigmosceptrella, Lacuntrulia, Diacarnus or Mycale genera belonging to the demospongiae class. Although most of these compounds are closely related, they bear different names depending the species or the genus of the sponge from which they were isolated. All these molecules bear a propionic acid function adjacent to the 1,2-dioxolane ring at one side and generally a sesquiterpene chain at the other side. Major differences between all these natural products, beside the relative stereochemistry, which can also be different, is the nature of the fatty chain. The farnesyl moiety can stay unchanged, such as in sigmosceptrellin E34 but can also give other types of cyclized structures: monocyclic (muqubilines A and B), ${ }^{35,36}$ bicyclic (trunculin A,37 mycaperoxide $\mathrm{A}^{38}$ ) or polycyclic structure by means of additional oxidative cyclizations (trunculin X). 39 These molecules were reported in general with moderated cytotoxic or antimicrobial activities, but have shown also some antiviral 38 and ichtyotoxic activities, ${ }^{40}$ which can explain their role as defensive metabolites for the sponges (Figure 3). 
A class of 1,2-dioxanes which can be considered apart, is the peroxyketals, where a methoxy substituent is generally exhibited. The oldest known marine endoperoxide is certainly chondrillin, isolated in 1976 from Chondrilla sp. sponge. 41 Some years later were isolated xestins from Xestospongia sp.. Xestin B exhibits the same 1,2-dioxene core structure than chondrillin and identical relative configuration; only the side chain being different with a diene framework..$^{42}$ Originally assigned absolute configuration for chondrillin was determined by circular dichroism, but revealed to be wrong, as demonstrated by total synthesis. 43 Therefore, chondrillin and xestin B finally share the same absolute configuration, which makes sense since their optical rotation sign is identical. Other 1,2-dioxanes belonging to the same class were also discovered. In one hand, peroxyacarnoate A or D were isolated from Acarnus $s p$. sponges,44,45 exhibiting an enyne framework on the side chain. On the other hand, manadoperoxide E46 and peroxyplakoric acid A147 were isolated from Plakortis $s p$. sponges. These two last substances exhibit a related side chain. Compared to peroxyacarnoates, some methyl substituents are present on the two last compounds. Manadoperoxides were reported to show a significant and selective antitrypanosomal activity (Figure 3). 


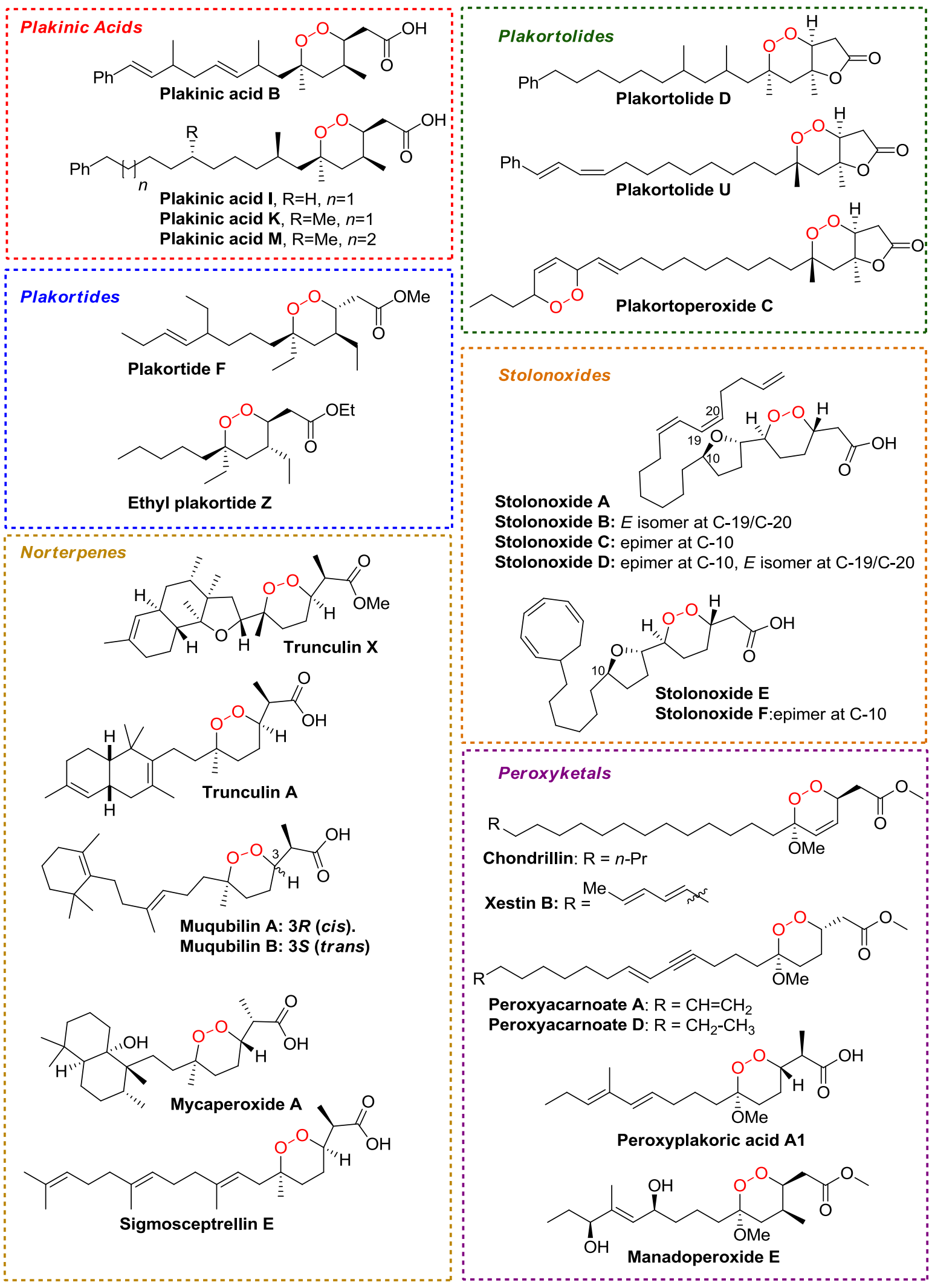

Figure 3. Illustration of different types of 1,2-dioxane carboxylate marine metabolites, through selected examples. 


\subsubsection{Other 1,2-dioxane or 1,2-dioxolanes}

Other endoperoxides can be found in various marine species, which bring sometimes very original molecules. Thus, sinugibberosides, 48 isolated from soft coral Sinularia gibberosa, provide an unprecedented 1,2-dioxanyl ketal along with an original bicyclo[7.2.0] undecane structure. From the same species was isolated peroxygibberol,49 which contains a 1,2-dioxene unit. Sinularia genus is also producing sinularioperoxide A and its different congeners, 50 which exhibit an endoperoxide structure. Dendiculatolide51 was isolated from soft coral Lobophytum denticulatum and exhibits an unusual 1,2-dioxane ring within a diterpenoid cembranolide-like macrocyclic structure. Ichthyotoxic activity was reported for this latter compound. Red soft coral Sarcophyton glaucum was providing sesquiterpenic dioxosarcoguaiacol,52 which possesses an original 1,2-dioxolanyl hemi-ketal framework. Peroxypolasol,53 structure isolated from the Japanese sponge Epipolasis sp., is a diterpenic endoperoxide with an original Lingshuiperoxide54 is also a sesquiterpene, which was isolated from the Hainan sponge Dysidea septosa. The 1,2dioxene certainly comes from a [4+2] cycloaddition with ${ }^{1} \mathrm{O}_{2}$, as well as the butenolide, which would be obtained from the same cycloaddition pathway on a furan ring, followed by a Kornblum-DeLaMare rearrangement. Intricated tricyclic structures gracilioethers A-C were isolated from Agelas gracilis,55 where gracilioethers A show a 1,2-dioxane ring instead of a cyclic ether. Other gracilioethers E-J were isolated from Plakinastrella mamilliaris, 56 which does not belong to demospongiae class, but to the homosclerophorida class. Some significant antimalarial and anti-leishmanial activities were reported for this family of molecules (Figure 4).

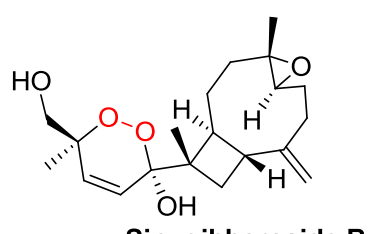

Sinugibberoside B

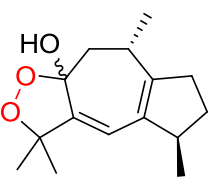

Dioxosarcoguiacol
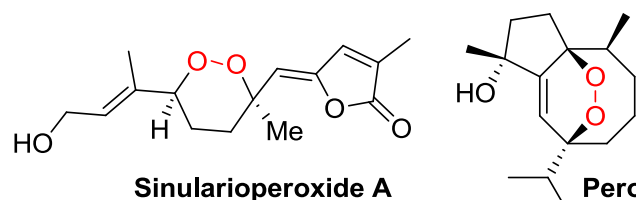

Peroxygibberol
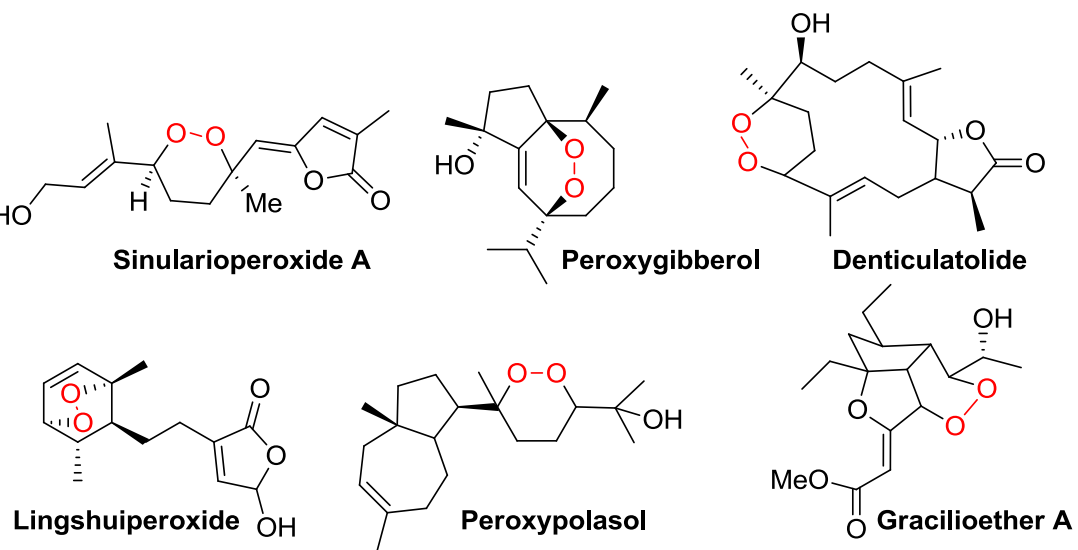
Figure 4. Structures of sinugibberoside B, sinularioperoxide A, peroxygibberol, dioxosarcoguiacol, lingshuiperoxide, peroxypolasol and gracilioether A.

\subsection{Terrestrial metabolites}

\subsubsection{1,2-Dioxolanes and 1,2-dioxanes isolated from plants}

Plants are also a major source of 1,2-dioxanes and 1,2-dioxolanes and most of these compounds are terpenoid-like products. Therefore, they can be classified according to their number of isoprene units.

Monoterpenes provide several examples of endoperoxides. In particular ascaridole was probably the first known and isolated natural endoperoxide. 57 This compound was originally isolated from epazote "Chenopodium ambrosioides", which is used traditionally in Mexican food to provide flavor, but also as infusion to improve digestion. The essential oil, which contains about 70\% of ascaridole, is reported to be toxic, but is useful for its anthelmintic properties. Acetylsaturejol and isoacetylsaturejol were isolated from Satureja gillesii, a Chilean lamiaceae. 58 The plant is resistant to herbivorous predators and the metabolites that it is producing are found to have significant toxicity toward shrimps Artemia salina. 1,2-Dioxolane and 1,2-dioxane structures are both isomeric, and might come from an ene reaction of singlet oxygen (see also paragraph 3.4.1) with pulegone or isopulegone (Figure 5).

Sesquiterpenes are the most representative class of endoperoxides in plants. Bisabolene-like yingzhaosu $\mathrm{A}^{59}$ and $\mathrm{C}^{60}$ were isolated from Artabotrys uncinatus, a traditional chinese herbal medicine for the treatment of malaria, and these compounds were particularly studied. Absolute and relative stereochemistry of yingzhaosu $\mathrm{C}$ was notably determined by total synthesis. ${ }^{61}$ Arteincultone $^{62}$ and spiroarteincultone ${ }^{63}$ were isolated from aerial parts of Artemisia maritima and/or Artemisia abrotanum. They are exhibiting a skeleton related to davanone, a sesquiterpenic tetrahydrofuran. A decahydronaphtalene-like structure can be seen in tirucaladerone, 64 a caladenequinone derivative recently isolated from Euphorbia tirucalli, as well as in schisansphene A,65 an eudesmene peroxy-derivative isolated from Schisandra sphenanthera. Eudesmanolide derivative tehranolide was isolated from Artemisia diffusa, ${ }^{66}$ and exhibits an unprecedented cyclooctane along with an endoperoxy hemiketal. Rugosal A,67 was isolated from leaves of Rosa rugosa and shows a carotane-like structure. This compound seems to act as an antifungal agent for the plant, which produces this compound when the leaves are damaged, in order 
to have a protection against some fungal infections. With a similar structure, isohalpinone is an example of guaiane-like natural endoperoxide, isolated from Alpinia japonica ${ }^{68}$ Another example, now in the guianolide family, is tanaparthin- $\alpha$ peroxide, isolated from Achillea setace, which exhibits an endoperoxide inside a cyclopentene ring (Figure 5).69

Diterpenes are also representatives of different 1,2-dioxanes. Texas broomweed Amphiachyris amoena afforded an original double spiro-1,2-dioxane with a labdane-type skeleton, called amoenolide $\mathrm{K}$. It was then proved experimentally that amoenolide A provides this latter compound by a ene reaction with singlet oxygen followed by a further oxo-Michael addition on a butenolide. $7^{\circ 0}$ African "Tonga croton" Croton steenkampianus Gerstner provided some years ago an unprecedented diterpenoic tetracyclic endoperoxide called steenkrotin B. ${ }^{71}$ However, a moderate anti-malarial activity was reported for this compound. In a recent total synthesis work trying to access to this natural product, the last cyclizing step, namely addition of hydroperoxide function to the ketone, was unsuccessful.72 Yet, the cyclization to the peroxyketal is supposed to be spontaneous, if favored, therefore assignment of the original structure of stenkrotin B can be questioning. Mulinic acid73 is also a diterpenic compound isolated from Mulinum crassifolium, a shrub growing in the north of Chile. Its structure was mainly determined by X-ray analysis, which secures its attribution. Internal 1,2-dioxene ring comes obviously from a [4+2] cycloaddition with singlet oxygen (Figure 5).

Some peroxy containing triterpenes were also found. For example, pseudolarolide $\mathrm{Q}_{2}$ was isolated from the seeds of Pseudolarix kaempferi, a chinese tree, and shows a complex 3(4),9(10)-disecocycloartane-type triterpene, occurring a 1,2-dioxolane ring. 74 Some taraxastane-type triterpenes are also known with an endoperoxide ring, such as $3 \beta$-acetoxy-1 $\beta, 11 \alpha$-epidioxy-12-ursene.75 This natural product was isolated from the aerial root of Ficus microcarpa, a Taiwanese ornamental plant and its elucidation was performed by X-ray crystallography (Figure $5)$.

Besides terpenes, other class of molecules can be isolated from plants, but there are more scarce examples. Some oxidized fatty acids possessing a 1,2-dioxolane such as thermalic acid A,76 could be found from the herbaceous plant Ophioglossum thermal, with potent antibacterial activity. Some alkaloids were found to possess endoperoxides such as catharoseumine, an isolated indolomonoterpene from 
Catharanthus roseus.77 Absolute configuration was determined by ECD and chemical degradation of the endoperoxide, in order to obtain a known natural product. This compound exhibited moderate antimalarial activity. Finally, some flavonoid glycosides, such as apigenosylide $\mathrm{B}$, are showing a spiro-1,2-dioxane. ${ }^{78}$ The main originality in its structure is the endoperoxide attached to the aromatic ring, which is quite unusual. This compound and other apigenosylides were found to display weak antibiotic and cytotoxic activities (Figure 5). 




Figure 5. Illustration of different 1,2-dioxanes or dioxenes and 1,2-dioxolanes isolated from terrestrial plants. 
Although plants are a major source of terrestrial endoperoxides, some of these type of compounds can be also isolated from fungus, bacteria or even animals.

Fungi are also a source of numerous endoperoxides, and in particular ergosterol peroxide, 79 originally isolated from Aspergillus fumigatus, is an ubiquitous endoperoxide, obtainable from many sources of mushrooms, but also from some plants, microorganisms and marine species. Some tricyclic sesquiterpenes can also be isolated from different species of fungi, with a structure close to talaperoxide D, isolated from Talaromyces flavus. ${ }^{80}$ Some anti-angiogenic activity and moderate cytotoxic activity were reported for this class of compounds. ${ }^{80,81}$ An unprecedented spiroketal peroxide, chloropupukeanolide A, was isolated from Pestaloptiosis fici, an endophytic fungus.82 This compound displays some significant anti-HIV and cytotoxic activities (Figure 6).

Mycangimycin is an unprecedented polyenic fatty acid bearing a 1,2-dioxolane ring. By some aspects, its structure is related to plakinic acids (paragraph 2.1.1), but the major difference is the absence of methyl substituents on the ring and the side chain. This natural product was isolated from Streptomyces strains, which are living in mutualism with an insect, the south pine beetle (Dendronctonus frontalis). Mycangimycin is produced to protect the insect against antagonist fungi, therefore this natural substance showed powerful antifungal activities, but also a strong antimalarial one (Figure 6). 83,84

Animals can be also at the origin of the production of important endoperoxides such as prostaglandins $\mathrm{H}_{2}$ or $\mathrm{G}_{2}$. These molecules are primary products of radical oxidation of arachidonic acid, and provide from different series of reactions including a Kornblum-DeLaMare rearrangement, all other prostaglandins, but also tromboxanes and prostacyclins. ${ }^{85}$ Prostaglandin $\mathrm{H}_{2}$ is not only a biosynthetic intermediate, but also displays some specific biological roles (Figure 6). ${ }^{86}$ 


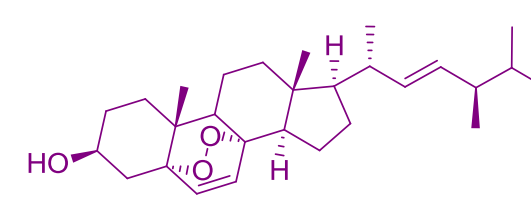

Ergosterol peroxide

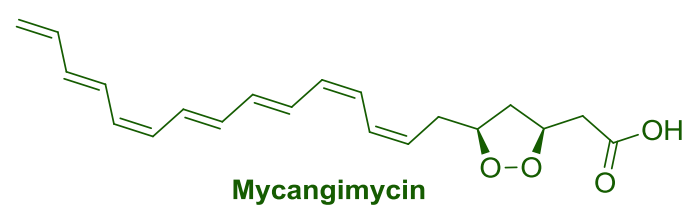

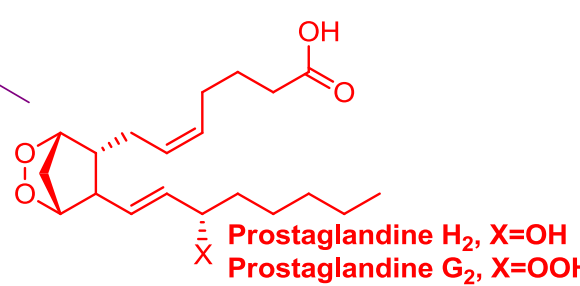

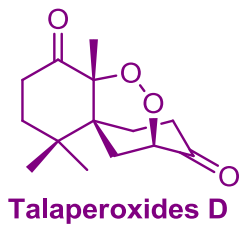

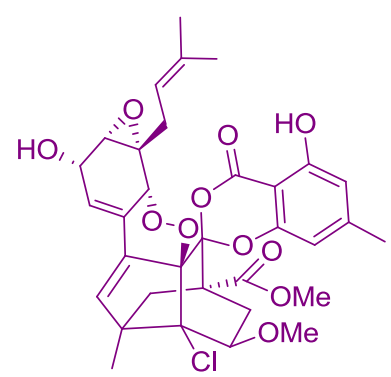

Chloropupukeanolide A

Figure 6. Examples of different endoperoxide metabolites isolated from fungi (purple), bacteria (green) and animals (red).

\section{Methods to synthesize 1,2-dioxolanes, 1,2-dioxanes and 1,2- dioxenes.}

\subsection{General considerations.}

Although it exists many methods to make organic peroxides and endoperoxides, such as 1,2-dioxolanes, 1,2-dioxanes and 1,2-dioxenes, the reagents allowing to make such structures are limited. Indeed, the direct formation of an $\mathrm{O}-\mathrm{O}$ bond is not really possible, since this bond is weak, alkoxy radicals cannot merge into peroxide bonds (the hemolytic cleavage is mostly observed) and since electronegativity of oxygen is also high, unpolung at oxygen atom is nearly impossible; only one example was reported using elemental fluorine, through the formation of a hypofluoride intermediate. 87 Thus, the only available reagents are: oxygen, which in its stable triplet state promotes mainly radical reactions, while more reactive singlet oxygen is able to provide a source of electrophilic oxygen and promotes cycloaddition reactions, and finally hydrogen peroxide as well as some hydroperoxides derivatives, which can provide a nucleophilic source of organic peroxides. A fourth reagent, which can be used in some specific application is $\mathrm{O}_{3}$, which procures hydroperoxyketals or acetals from olefins in presence of a protic solvent.

Therefore, the methods to synthesize 1,2-dioxolanes, 1,2-dioxanes and 1,2dioxenes will be classified depending their method of preparation. Indeed to make an endoperoxide, the elaboration of two $\mathrm{C}-\mathrm{O}$ bonds are in general needed to introduce the peroxide unit and build the ring. The two bonds can be established either in one step when the two $\mathrm{C}-\mathrm{O}$ bonds are formed in a chain reaction (particularly with free 
radical reactions) or in a concerted fashion (cycloadditions), or in two steps when first the construction of a hydroperoxide or a derivative thereof is first necessary followed by its cyclization (Figure 7).

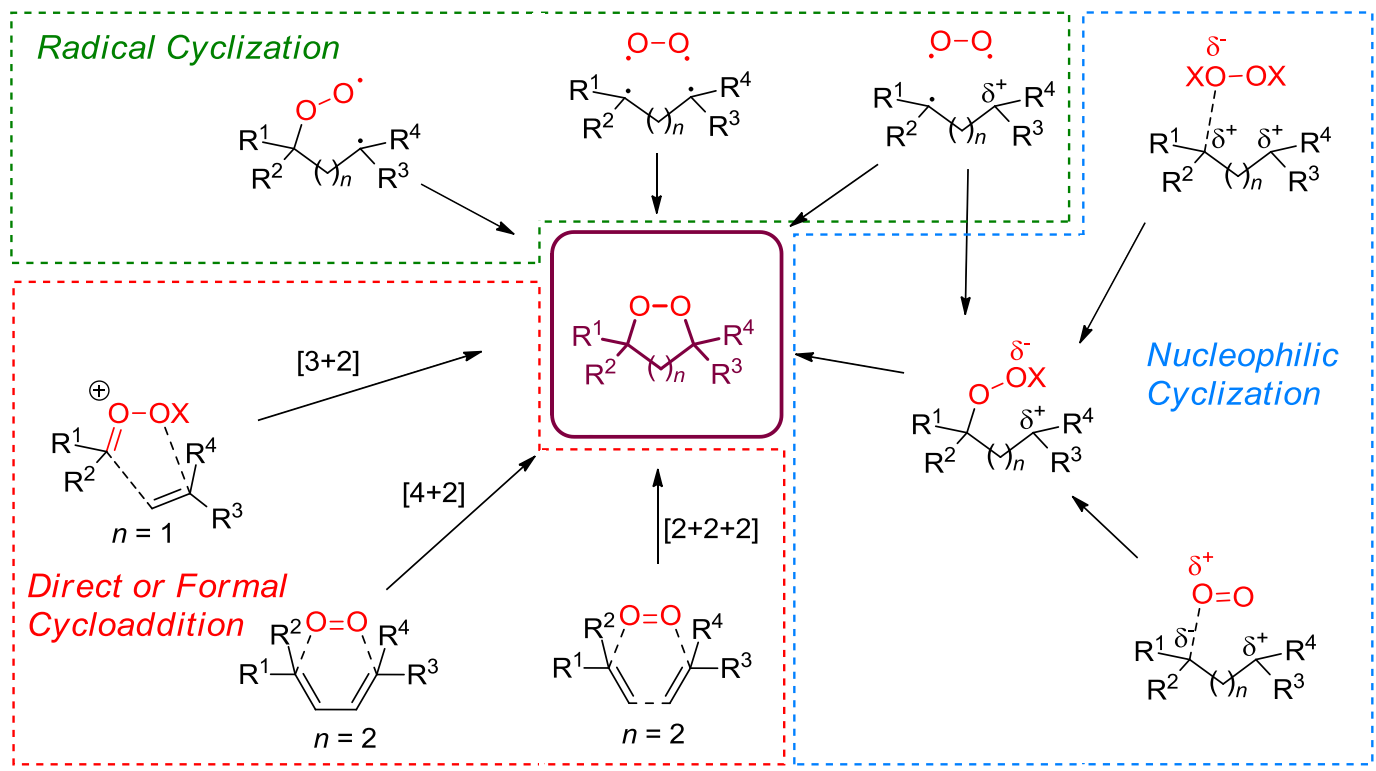

Figure 7. Different strategies and pathways to build 1,2-dioxanes and 1,2-dioxolanes.

\subsection{Radical peroxidations and peroxycyclization.}

\subsubsection{Radical addition of triplet oxygen and direct formation of an endoperoxide.}

\subsubsection{Cycloaddition of triplet oxygen onto diradical species.}

The direct formation of endoperoxide is a possible process through the formation of a diradical species. Thermal decomposition of cyclic azo compounds was early explored in the 80's under oxygen atmosphere and afforded 1,2-dioxanes and/or dioxolanes. 88 An original approach was conducted some years later and consisted in the decomposition of a bicyclic azo compound under light irradiation with an argon laser at $351.1,351.4$ and $363.8 \mathrm{~nm} .{ }^{89}$ The goal was to make some analogs of prostaglandin $\mathrm{H}_{2}$. The authors started by introducing hydrazide framework $\mathbf{3}$ by using a [4+2] cycloaddition between azo compound $\mathbf{1}$ and cyclopentadiene 2. Further functionalization of the olefin and introduction of a side chain, followed by the elimination of the imide moiety afforded azo compound 4 . At this stage, application of the laser argon in Freon at $-16{ }^{\circ} \mathrm{C}$ under oxygen afforded expected diastereomers 6a (endo) and $\mathbf{6 b}$ (exo) in modest yields after 
chromatography at $-16{ }^{\circ} \mathrm{C}$. Diradical species 5 was reported to be the key intermediate (Scheme 1).



\section{Scheme 1}

Latent diradicals are available from some specific dienic systems. An interesting example is illustrated from flash vacuum pyrolysis of furan 7 , which led to the formation of non-aromatic compound 8. A reorganization of electrons probably took place with recovery of aromaticity as a driving force and formation of diradical species 9. It is then involved in the formation of 1,2-dioxane 10 with triplet oxygen at low temperature. That is interesting to note this transformation led to the same compounds than those obtained using a [4+2] cycloaddition between a diene and ${ }^{1} \mathrm{O}_{2}$ (see paragraph 3.4.2), however it appears that the two transformations are completely different mechanistically speaking (Scheme 2).90

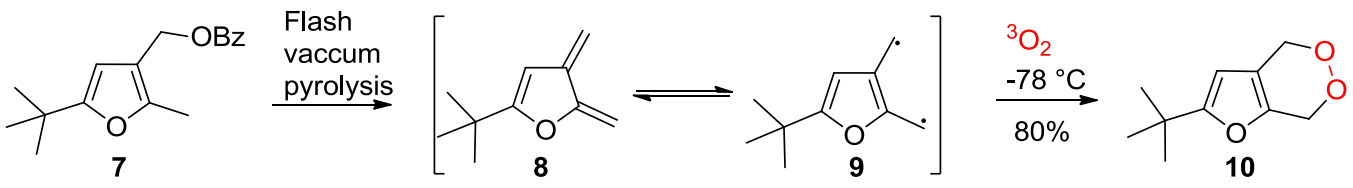

\section{Scheme 2}

\subsubsection{Formal cycloaddition of triplet oxygen onto radical cations}

Another method to prepare some endoperoxides is the triplet oxygen peroxidation of cation radicals. Original works on this process were using an appropriate one-electron oxidizing reagent, such as dicyanoanthracene with photoactivation. This process was only reserved to electron-rich cyclopropanes, or dienes for example. Otsuji and Mizuno's pioneer work demonstrated that an oxidation through a single electron transfer takes place onto diarylcyclopropane such as product $\mathbf{1 1}$ in order to lead to 1,2-dioxolanes. ${ }^{11}$ Presumably oxidation occurs on the heteroatom (12) and lead by conjugation to the opening of the strain cyclopropyl ring 
(13). Stabilized radical $\mathbf{1 3}$ is then reacting with triplet oxygen (14) and further cyclization of the peroxyradical drives to rearomatization to radical cation $\mathbf{1 5}$. Doubtless, an electron transfert from starting molecule $\mathbf{1 1}$ takes place in order to propagate the reaction and furnish 1,2-dioxolane 16. Addition of $\mathrm{Mg}\left(\mathrm{ClO}_{4}\right)_{2}$ was reported to dissociate dicyanoanthracene radical anion from the radical cation leading to an overall acceleration of the process (Scheme 3).91

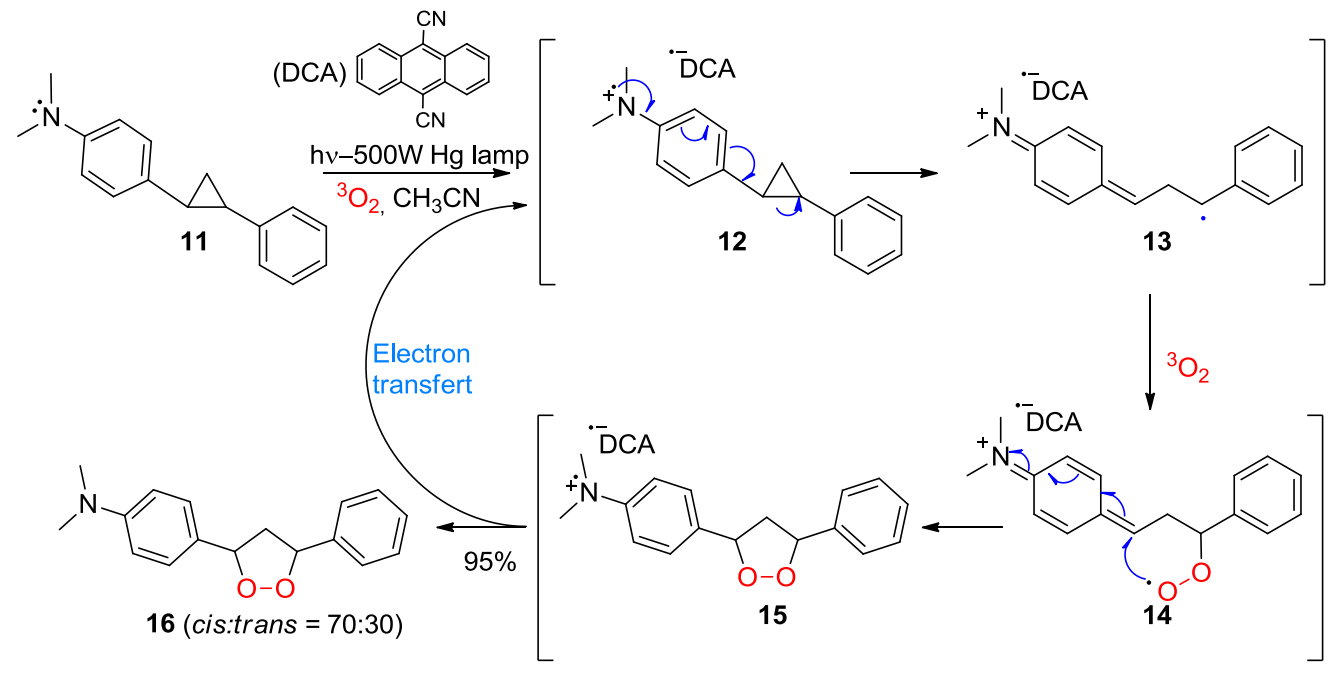

Scheme 3

Research of a more applicable process showed that $\mathrm{TiO}_{2}$ could be used in place of dicyanoanthracene.92 More recent work of Yoon and coworkers showed also that $\mathrm{Ru}(\mathrm{bpz})_{3}{ }^{2+}$ (19) is a more effective catalyst than dicyanoanthracene, mainly by performing the ring expansion with simple visible light apparatus at low temperature and with a wider scope of substrates (Scheme 4). 93

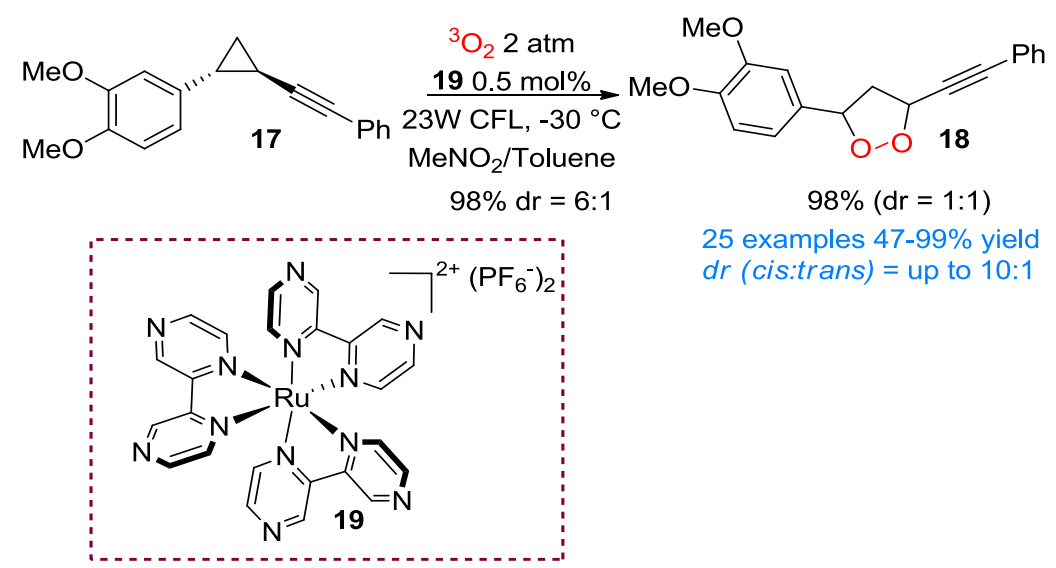

Scheme 4

This process was also applicable to the synthesis of 1,2-dioxanes. A pioneer example was reported by the same authors on electron-rich conjugated dienes, 
spaced by 3 or 4 atoms. On substrate $\mathbf{2 0}$, photooxidation with dicyanoanthracene leads to the formation of cationic radical 21, which can cyclize on the other double bond to drive to species 22. Further reaction with $3 \mathrm{O}_{2}$ drives to the formation of peroxy radical $\mathbf{2 3}$ and cyclization to endoperoxide 24. Electron transfer from $\mathbf{2 4}$ to 20 propagates the reaction furnishing 1,2-dioxane 25. This transformation can be viewed as a formal $[2+2+2]$ cycloaddition. The presence of two aryl substituents for each olefin appears to be crucial, but limits the scope of the reaction (Scheme 5).94

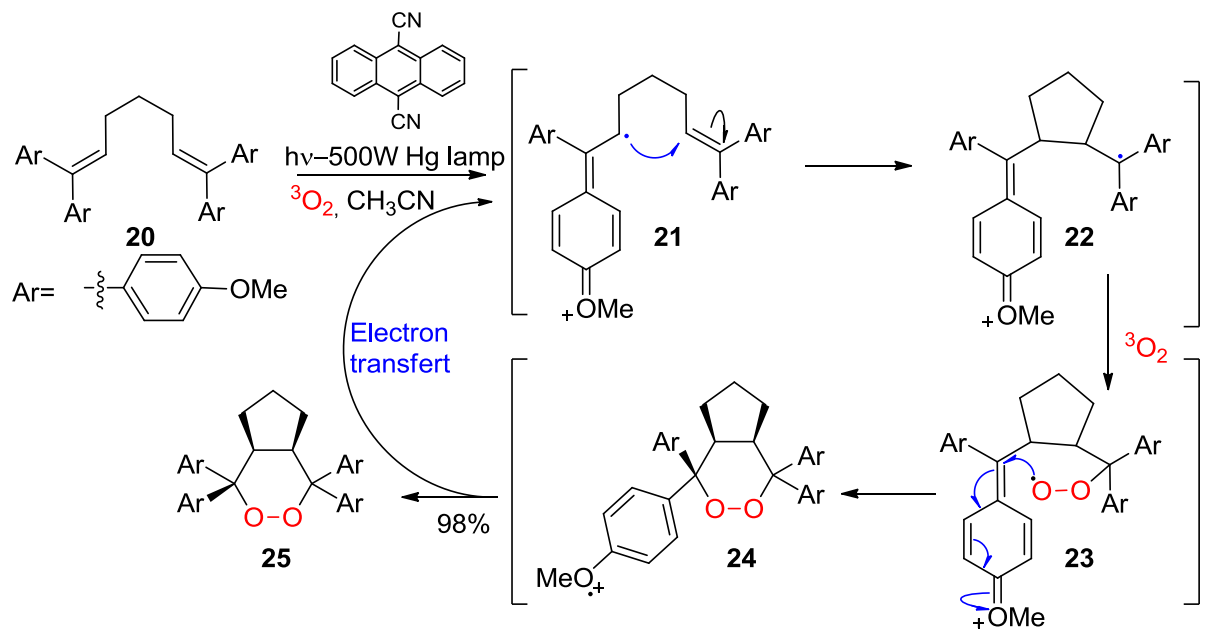

Scheme 5

Fortunately, almost 20 years later was reported an updated protocol by Yoon and coworkers.95 They described a similar process on monosubstituted olefins. Particularly, they showed that tris(bipyrazyl)ruthenium(II) complex 19 proved to be a very suitable photosensitizer for such a transformation because of the far superior excited state lifetime. Some years later, Nicewicz and coworkers presented a similar work based on photoexcitation with pyrilium species 26.96 They demonstrated that the presence of an electron-donating group was crucial through substrate screening, and they applied the reaction mainly to 1,1-disubstituted olefins. An example leading to tricyclic structure $\mathbf{3 0}$ was particularly interesting. More recently was reported a study using $\mathrm{TiO}_{2}$ nanoparticules as photosensitizer by Wang and coworkers (Scheme 5).97 Although Yoon and coworkers obtained under some conditions some cyclobutanes such as compound $\mathbf{3 1}$ as byproducts, it appears that this strained cycle is probably a reversible intermediate formed when oxygen is not reacting, but can nevertheless lead to 1,2-dioxane 32 when placed in photooxygenation conditions. Although previous works mentioned that the photosensitizers used do not produce 
any superoxide ion, Wang and coworkers' study showed some evidences of the role of superoxide anion in the formation of dioxanes with $\mathrm{TiO}_{2}$ as photocatalyst (Scheme 6).

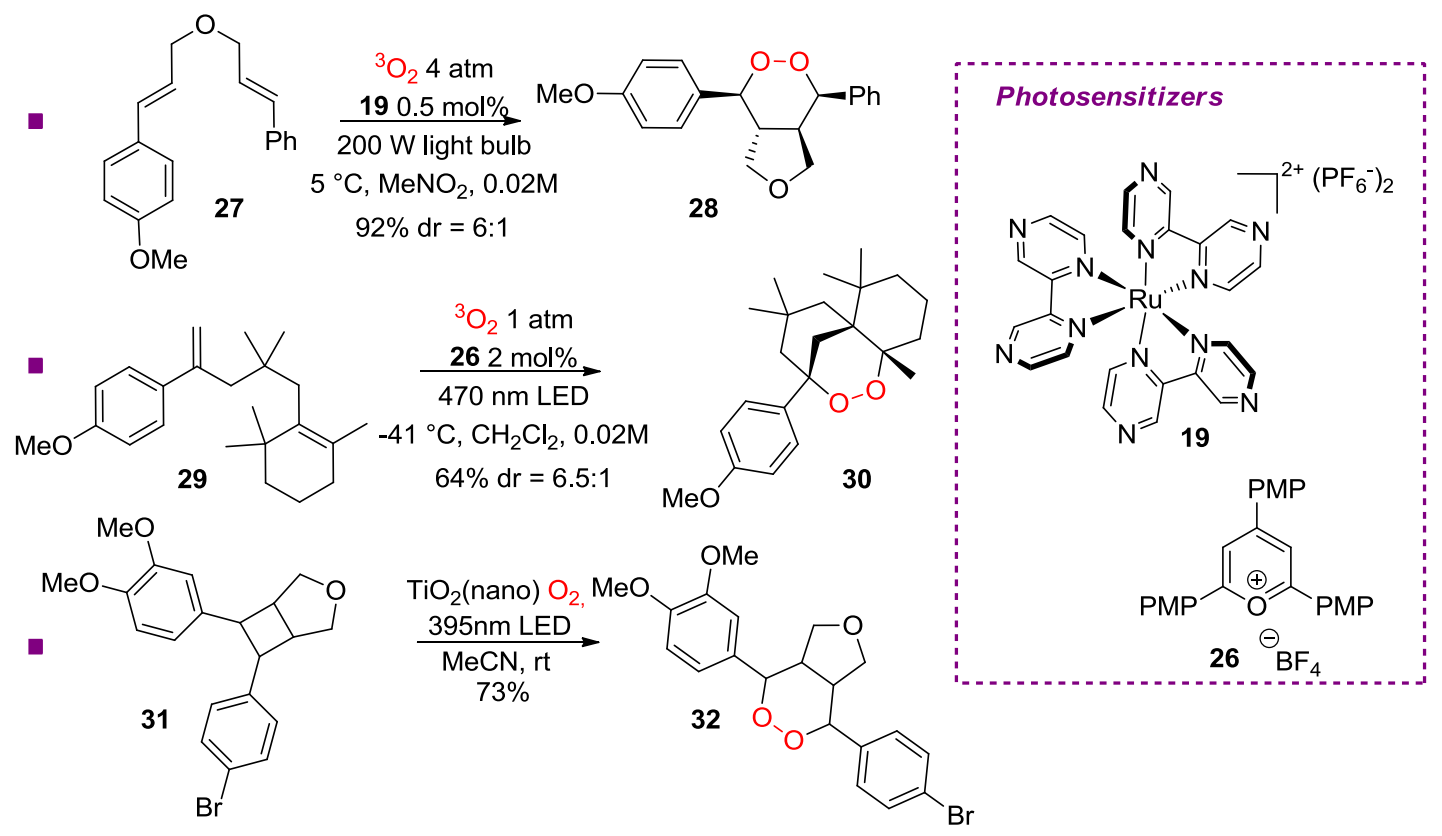

Scheme 6

Using a similar mechanism, some interesting work was developed on cyclopropylamines. Wimalasena and coworkers found that cyclopropylaniline derivatives can react in a single electron oxidation. 98 Therefore cyclopropylamine $\mathbf{3 3}$ could react in presence of air with different reagent to obtain 1,2-dioxolanes $\mathbf{3 6}$. The mechanism is similar to the one depicted in scheme 3 , through oxidation and formation of radical cation $\mathbf{3 4}$ on the nitrogen, followed by the ring-opening of the cyclopropyl ring by the radical. Further reaction with oxygen lead to 1,2-dioxolane 36. Because of the instability of these endoperoxides, no yield could be given, even if some NMR spectroscopic evidences were obtained. The instability is probably due to the nitrogen, which possess some basic and nucleophilic properties driving to some degradation such as a Kornblum-DeLaMare rearrangement. Independently, Six and coworkers noticed the formation of an endoperoxide from the reaction mixture of the intramolecular Kulinkovich-DeMeijere reaction of amide 37.99 Instead of isolating expected cyclopropylamine $\mathbf{3 8}$, dioxolane $\mathbf{3 9}$ was obtained after purification, due to a spontaneous oxidation with air. Presumably the electron donating aromatic group on nitrogen facilitated this process. Encouraged by this interesting result, they found that anodic oxidation of cyclopropylamines under oxygen drove to 1,2-dioxolanes with a better variety of substrates such as compound $\mathbf{4 0}$ converted into endoperoxide 41. ${ }^{100,101}$ A vinylogous process was also explored from some piperidine derivatives 
and proved to be effective. ${ }^{102}$ As described by Wimalesena and coworkers, the main difficulty in this study was the instability of the amino-dioxolanes. Only few examples were found to be stable and isolable. Substitution increases stability giving a favorable Thorpe-Ingold effect (Scheme 7).

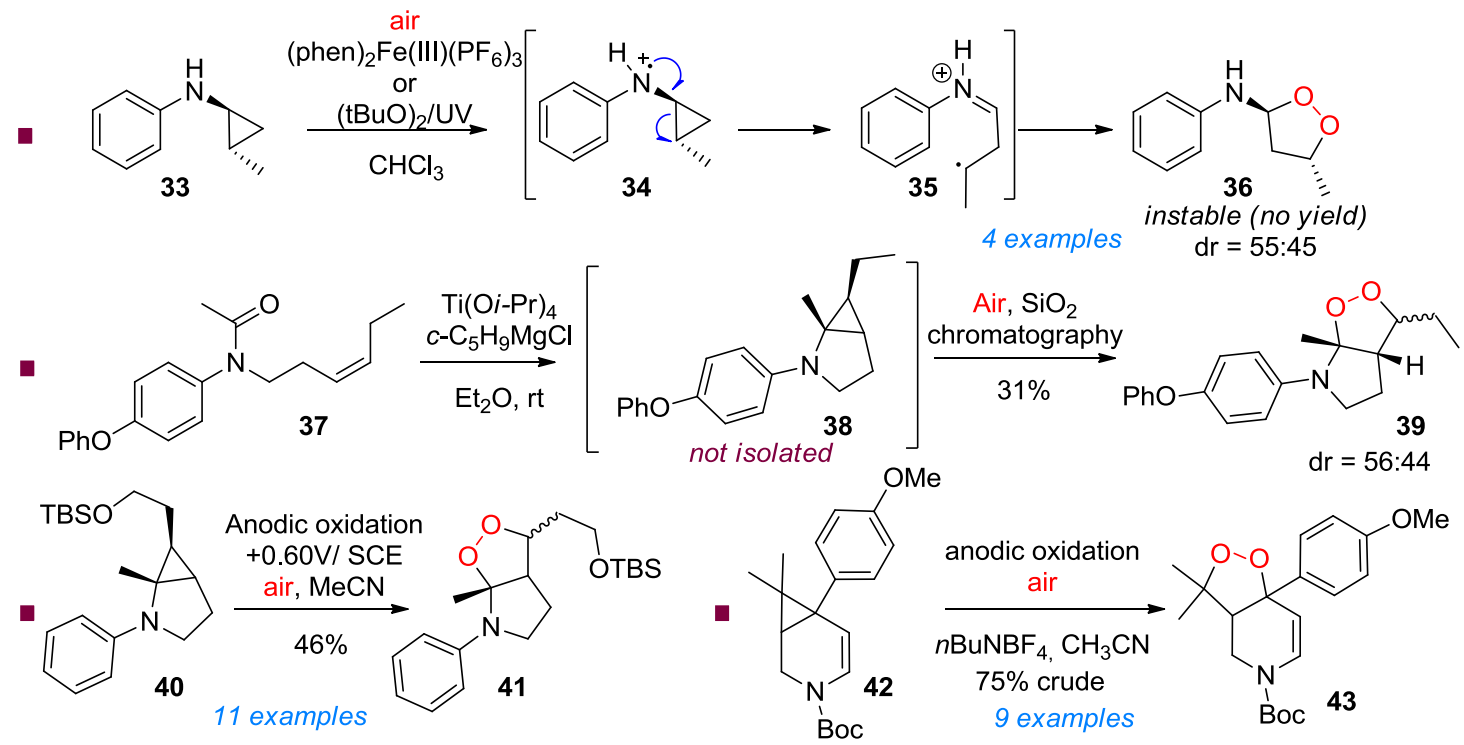

Scheme 7

A very recent example of Zhou and Lu seemed to use a similar mechanical pathway for the aerobic oxidation of spirovinyl-cyclopropylindoles mediated by some iodine. ${ }^{103}$ Thus, compound $\mathbf{4 4}$ was, for example, converted into 1,2-dioxolane 45 in 91\% yield with air and 1 mol\% of $I_{2}$. Some control experiments showed a radical mechanism for this transformation, since photons seem necessary to activate the reaction and the addition of radical inhibitors stops the process. Most of the studied substrates, leading to effective transformations, showed only differences on the substituents on the aromatic ring, which unfortunately limits the scope of this reaction. A plausible mechanism could be firstly the reaction of the nucleophilic amide function in $\mathbf{4 6}$ onto iodine to make an I-O bond. Intermediate 46 undergoes then a homolytic cleavage, probably by activation with light irradiation, giving radical cation $\mathbf{4 7 a}$ in mesomerism with $\mathbf{4 7} \mathbf{b}$. The free radical opens then the strained ring by a conjugated process, and radical species $\mathbf{4 8}$ reacts then with oxygen to obtain peroxy radical 49, which can cyclize into 1,2-dioxolane 50. Electron-transfer from 50 to starting material $\mathbf{4 4}$ can then propagate the reaction (Scheme 8). 


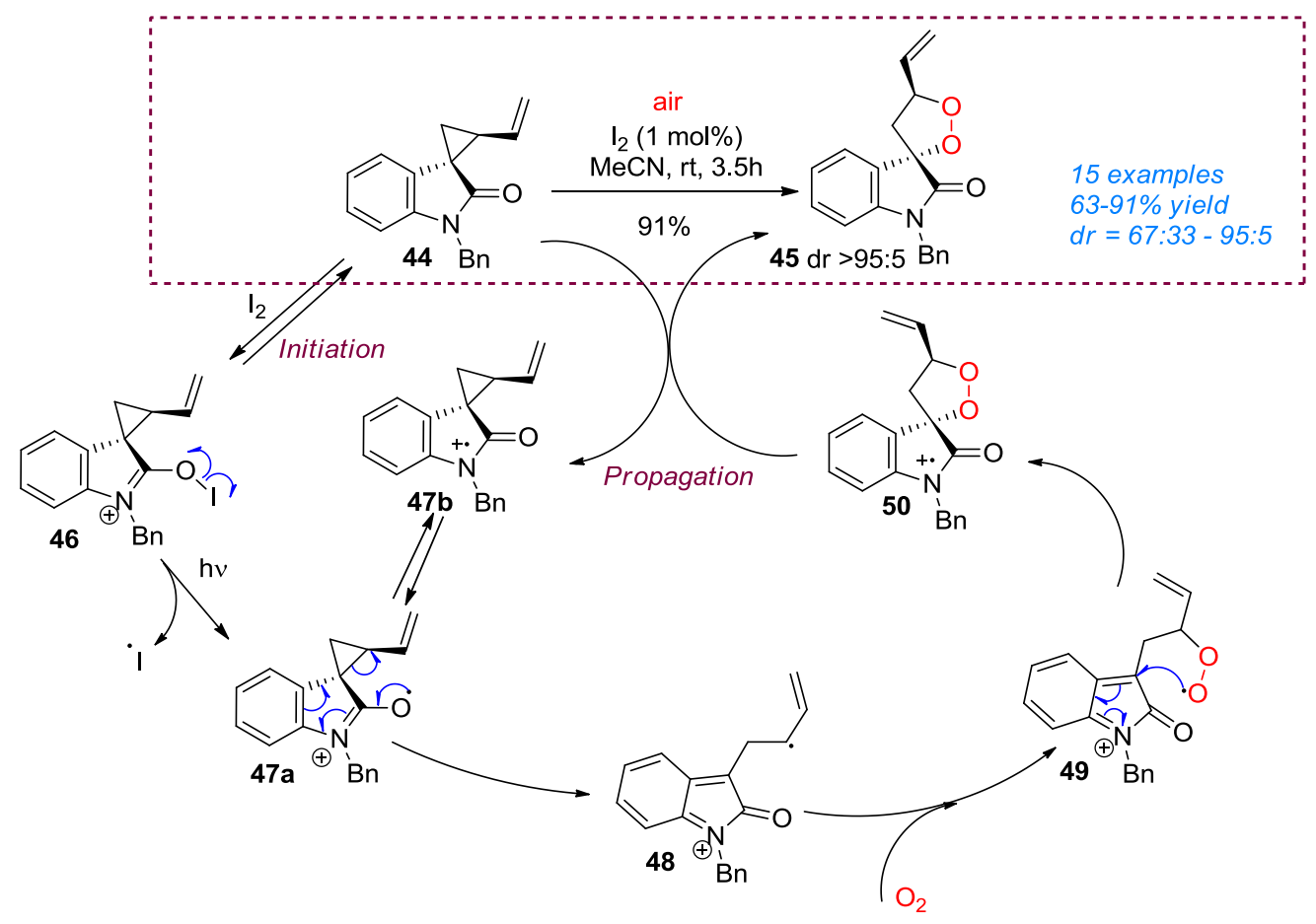

Scheme 8

A very recent example of cycloaddition of oxygen triplet on annellated 1-aryl-1carboxyl-cyclopropane derivatives 51a-k, mediated by visible light in presence of acridine derivative such as [MesAcr $] \mathrm{ClO}_{4}$ as sensitizer, was also described by Reiser and coworkers to give fused 1,2-dioxolanes.104 Diverse structure were obtained, mainly some THF derivatives such as 52a-e in modest yields; pyrrolidine or piperidine derivative $\mathbf{5 2 f}$ and $\mathbf{5 2 g}$; and cyclopentenyl derivatives $\mathbf{5 2 h}-\mathbf{i}$. The reaction was in contrast ineffective when no aromatic ring was present on the cyclopropyl group and also to prepare tetrahydropyrane derivative $\mathbf{5 2 k}$. Limitations were also observed when cyclohexenyl rings were used in place of cyclopentenyl rings. (Scheme 9) 

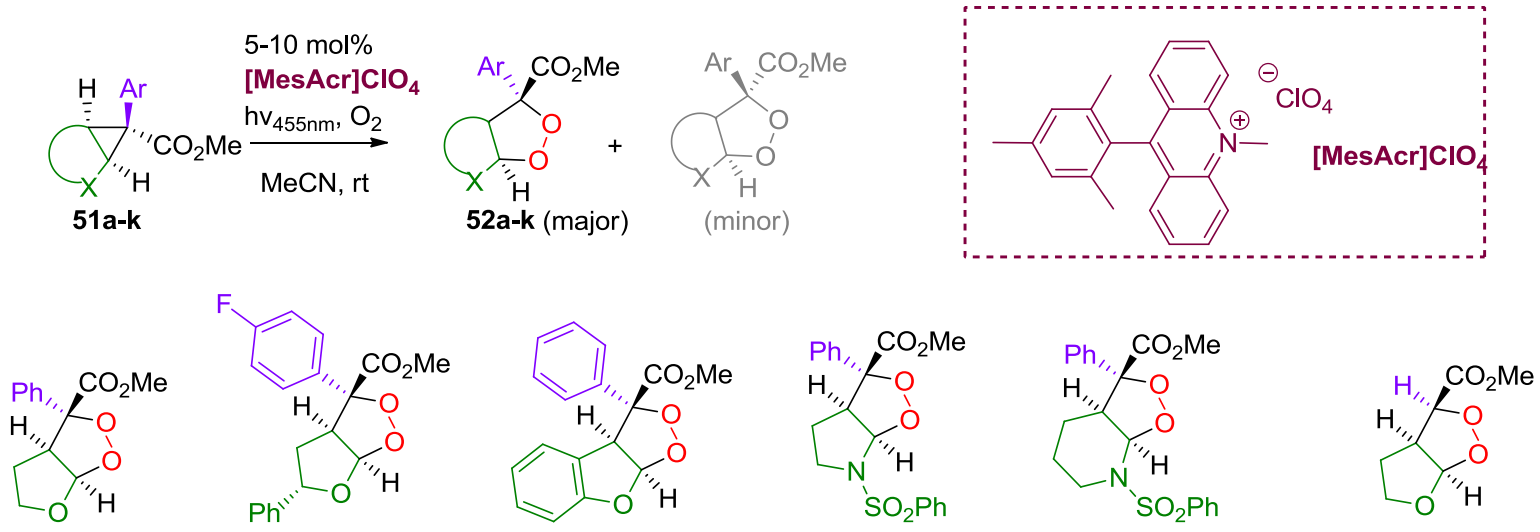

52a $47 \%, d r=4: 1$

52b $47 \%, d r=3: 1$

52c $60 \%, d r=4: 1$

$52 f 34 \%, d r=1: 1$

52g $37 \%, d r=5.3: 1$

52j $0 \%$,No Reaction

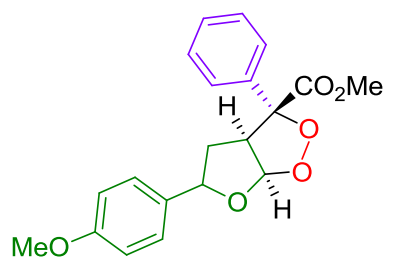

52d $68 \%, d r=3: 1$



52e $45 \%, d r=4: 1$

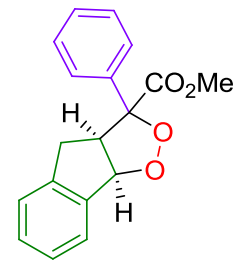

52h $59 \%, d r=2: 1$

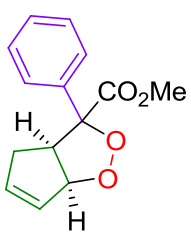

52i $63 \%, d r=99: 1$

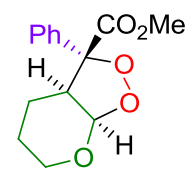

52k 0\%,No Reaction

Scheme 9

\subsubsection{Formal cycloaddition of triplet oxygen to cycloalkoxy} radicals

Although the spontaneous reaction of 1,2,2-trimethylcyclopropanol with oxygen into 1,2-dioxolane was reported early in 1969, ${ }^{105}$ a more general protocol was developed much later in 2001 by Kulinkovich and coworkers, where they showed Manganese (II) abietate (a diterpene carboxylate) in benzene is able to initiate the ring expansion of cyclopropanols 53. ${ }^{106}$ Presumably a superoxomanganese(III) species is involved in the reaction and can abstract an hydrogen from cyclopropanols 53 to form alkoxy radicals 54, which can freely evolve into an alkyl radical species $\mathbf{5 5}$. This latter can then trap a molecule of oxygen, and a cyclization followed by a propagation process (the two steps probably can work one way or the other) lead peroxy radicals species 56 to the formation of 3-hydroxy-1,2-dioxolanes 58 . These compounds were described to be in equilibrium between the open hydroperoxyketone form and endoperoxyde form, leading to thermodynamic mixture of epimers at peroxyacetal function. The usefulness of this heterocycle was neglected at this time (see paragraph 3.5.2), and its transformation into epoxyketones 59 was mainly achieved without characterization of 1,2-dioxolane intermediate 58 . (Scheme 10)

Similarly, a more recent work showed that tertiary cyclopropanols $\mathbf{6 0}$ could react in almost quantitative yield in THF with manganese(III) acetylacetonate to 
obtain 5,5'-unsubstituted 1,2-dioxolanes $\mathbf{6 1}$ in order to prepare chiral epoxyketones 62 with poly-L-leucine matrix. ${ }^{107}$ (Scheme 10)
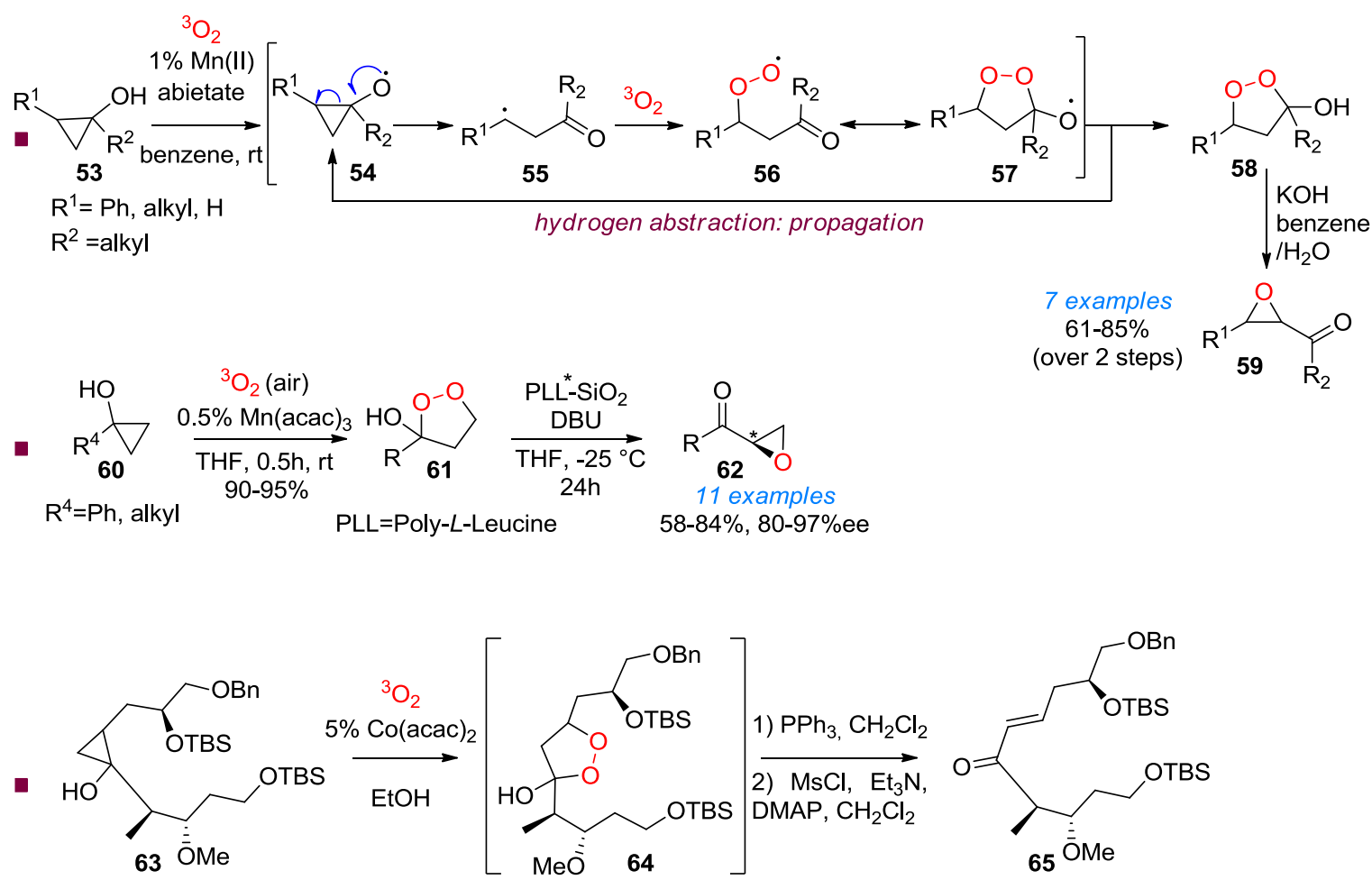

not isolated 14 examples $69-92 \%$ (over 3 steps)

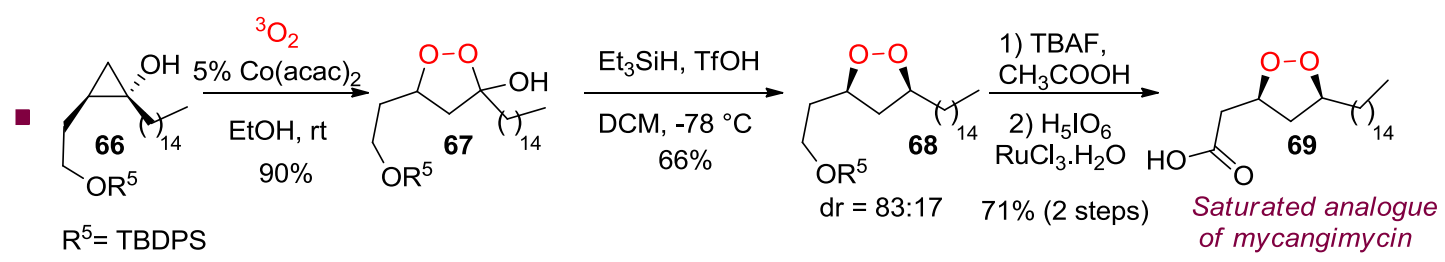

Scheme 10

In another example, cyclopropanol 63 could also be readily opened under radical conditions with cobalt(II) acetylacetonate, the mechanism of which is similar to that with manganese. The reaction was originally reported in ethanol with a higher metal charge (5 mol\%). Such as previous examples, 1,2-dioxolan-3-ol 64 was not targeted but used as a reactive intermediate for further transformation. Indeed, authors transformed endoperoxide 64 into conjugated ketone 65 by reduction of the peroxide bond with $\mathrm{PPh}_{3}$ followed by elimination of the hydroxyl group after mesylation. Fourteen examples on poly-functionalized structures were reported with yields given between 69 and $92 \%$ over a 3 step sequence. ${ }^{108}$ (Scheme 10)

A last example reported by us was the utilization of this radical reaction in the synthesis of saturated analogue of mycangimycin 69 (see Figure 6, paragraph 2.2.2). 
The ring expansion was carried out from cyclopropanol 66 with $\mathrm{Co}(\mathrm{acac})_{2}$ in ethanol and led to 3,5-disubstituted dioxolane $\mathbf{6 8}$ after reduction of the hydroxyl group with triethylsilane (see also paragraph 3.5.2). ${ }^{109}$ (Scheme 10)

The driving force of these previous transformations is unambiguously the energy strain contained in the cyclopropyl ring, which lead to the formation of an alkyl radical where a peroxidation can take place. Considering cyclobutanes are also strained rings, we recently showed that he radical ring opening of cyclobutanols 7oai might drive to the formation of 1,2-dioxan-3-ols 71a-i through a formal ring expansion process. ${ }^{110}$ The process is much slower than with cyclopropanols (Scheme 7) and required higher cobalt salt load and higher reaction time to proceed. The reaction is working particularly well with secondary alcohol such as with cyclobutanols 7oa-b, 70e-f, or 7oh, and is more sluggish when it is substituted by an alkyl group such as with $\mathbf{7 0 b - c}$, or $\mathbf{7 0 g}$. In contrast the substitution at position 2 improves the stabilization of the reactive radical species, which increased the kinetic of the reaction and led to good yields even at room temperature such as with $\mathbf{7 0 a - b}$, 7og, 70i. (Scheme 11)

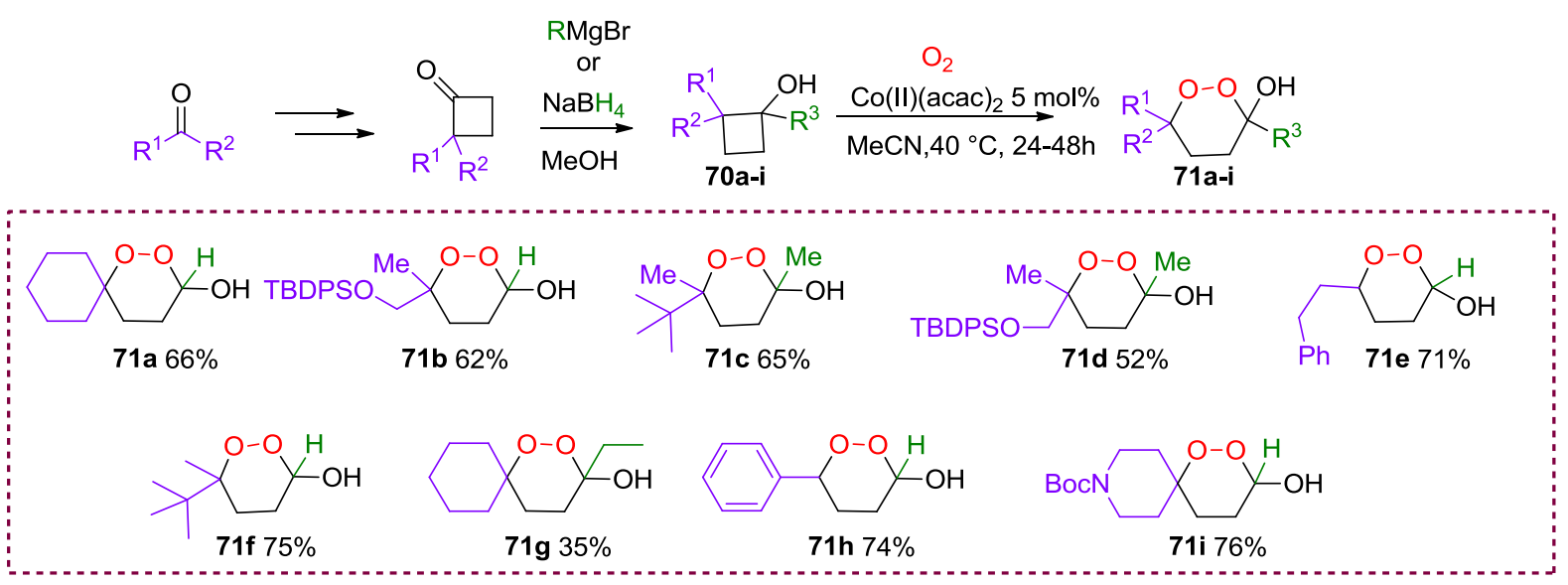

\section{Scheme 11}

Although larger ring sizes, such as 5 or 6 member rings, are in general not appropriate to undergo such radical cleavage, some specific examples were reported in the literature about rearrangement of some steroid derivatives in the manner of a Wharton-like fragmentation, but under radical conditions. Thus, Suarez and coworkers discovered a radical fragmentation of steroids containing a cyclopropane ring, mediated by $\mathrm{PhI}(\mathrm{OAc})_{2}, \mathrm{I}_{2}$ and some light. Following authors' description, decalin ring-opening of compound $\mathbf{7 2}$ into dioxolane $\mathbf{7 3}$ was generated from the formation of alkoxyradical 74. Fragmentation of the decalin structure followed by 
cyclopropyl ring-opening led to 1,2-dioxolane 77 after reaction with one molecule of oxygen. The remaining alkyl radical reacted with an additional molecule of oxygen, and underwent several transformations, whom a hydrogen atom transfer, to form THF ring present in product $\mathbf{7 3}$. However the authors are evasive concerning this last transformation step, therefore the obtaining of THF ring was not completely understood (Scheme 12). ${ }^{111}$

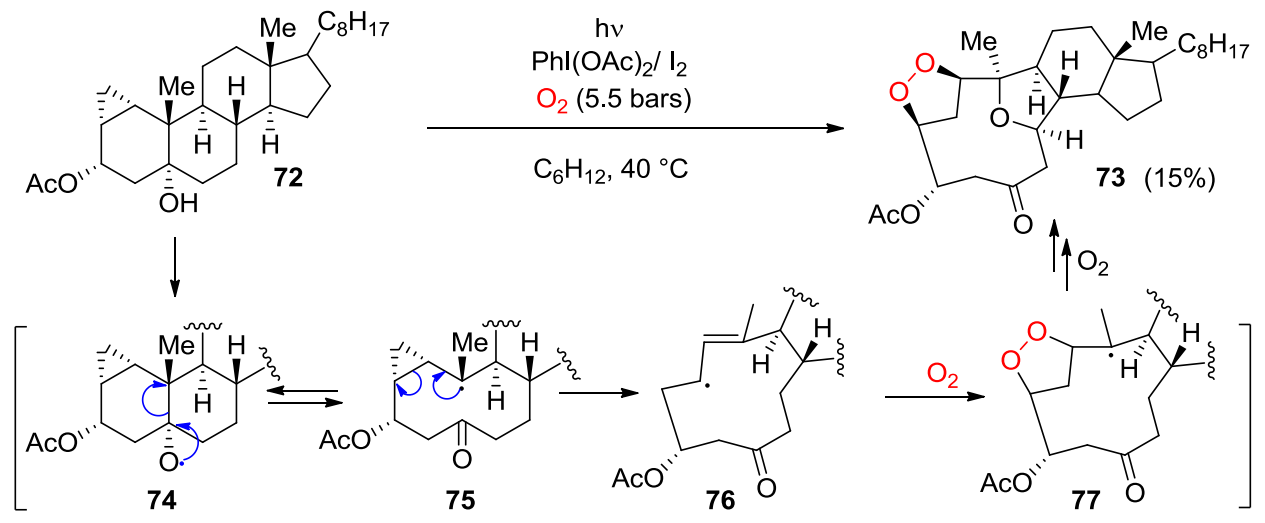

Scheme 12

3.2.1.4 Formal [2+2+2] cycloaddition of triplet oxygen to enoxy radicals and olefins

Besides the ring opening of cycloalcanols through an alkoxy radical, which can lead to endoperoxides, enols were particularly well studied to promote some formal $[2+2+2]$ cycloadditions with triplet oxygen and a good radical acceptor such as some styrene derivatives. ${ }^{112}$ Thus in a seminal work, cyclohexanone $\mathbf{7 8}$ was reported to react with styrene $\mathbf{7 9}$, oxygen and a combinaison of $\mathrm{Co}(\mathrm{II})(\mathrm{OAc})_{2} \& \mathrm{Mn}(\mathrm{II})(\mathrm{OAc})_{2}$ at $80{ }^{\circ} \mathrm{C}$ to give specifically 1,2-dioxan-3-ol 80 (Scheme 13).

The reaction was then more generalized on 1,3 dicarbonyl systems with at least a ketone as function and $\mathrm{Mn}(\mathrm{II})(\mathrm{OAc})_{3}$ as a radical initiator. Piperidone 81, for example, gave rise to endoperoxide $\mathbf{8 3},{ }^{113}$ whereas piperidinedione 84 afforded dioxanol 86. ${ }^{114}$ When a third carbonyl group is used as substituent, the reaction could afford tricyclic structures after a further cyclization. Therefore, spontaneous cyclization and dehydration of endoperoxyketal $\mathbf{8 8}$ gave compound $\mathbf{8 9}$ when a ketone was involved as a substituent, while an ester such as in structure $\mathbf{9 2}$ led to tricylic compound 93 containing a lactone. ${ }^{115,116}$ The mechanism of the reaction was reported with different hypotheses, particularly in the first mechanistic step of the reaction. It was observed that the limiting step is the formation of enol species 95. By consequence the easier enol $\mathbf{9 5}$ is formed, the faster the reaction is, meaning also 
enol species 95 is the reactive species compared to diketone 94. This also explain the importance of having a 1,3-dicarbonylated substrate to enhance the presence of enol species. Manganese(III) is then inserted on the hydroxyl group and undergoes an hemolytic cleavage to give alkoxy radical $\mathbf{9 7}$ at one side and a manganese(II) species at the other side. The formation of a radical cation was also proposed by some authors at this stage. ${ }^{117}$ The free radical shifts to the more stabilized alkyl position such as with species 98 and then reacts first with radical acceptor 99 (in general a styrene derivative), to give rise to alkyl radical 10o. In a second time, addition of triplet oxygen furnished 1,2-dioxane 102 after $\mathrm{H}$ abstraction (Scheme 13).

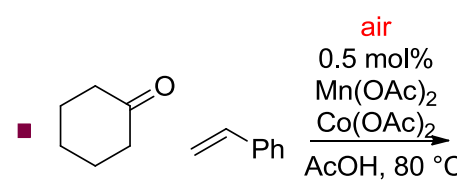

78

79

$70 \%$



80



12 examples $22-82 \%$

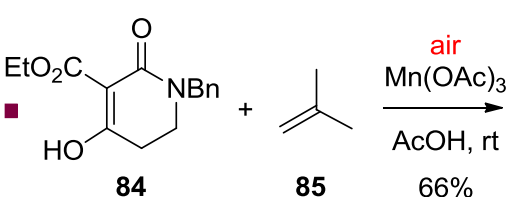

84

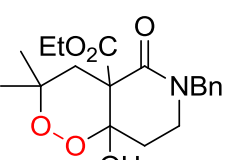

$86 \mathrm{OH}$

28 examples (17-93\%)<smiles>O=C(CC1C(=O)CC[NH+]([PH3+])C1=O)c1ccccc1</smiles>

87
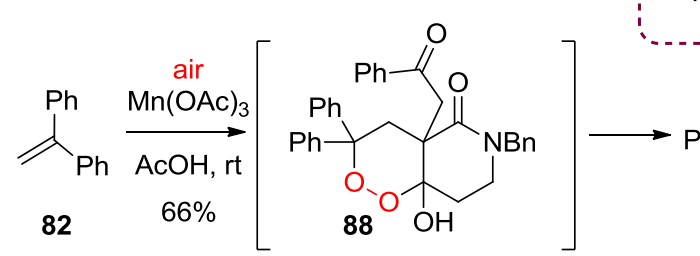

14 examples (19-75\%)
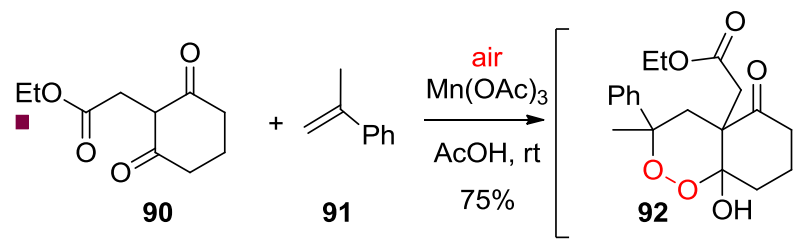

\section{General Mechanism}<smiles>[R]C(=O)CC([R])=[18O]</smiles>
$-\mathrm{AcOH}_{\alpha} / \mathrm{Mn}(\mathrm{III})$

$\mathrm{R}$<smiles>[R]C(=O)C=C([O-])[O-]</smiles><smiles>[R3]C(=C)[R](=[Z])C</smiles>
$\checkmark(\mathrm{OAc})_{3}$<smiles>[R]/C(=C/C(C)=O)OCCC</smiles>
$\mathrm{R}^{2}$<smiles>[R]C(=O)CC([R6])=O</smiles><smiles>[R]C(=O)C(C([R])[R])C([R])[R]</smiles>

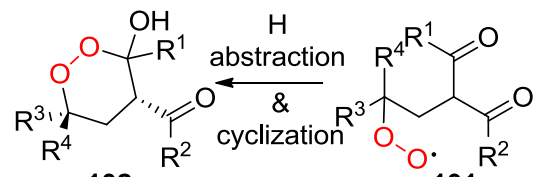

102 101

\section{Scheme 13}

This reaction was also applied to some heterocycles exhibiting a 1,3-dicarbonyl framework. Especially, quinolinols are preferentially existing as an enol due to a conjugation with the aromatic ring. Thus, compound 103 reacted with 
diphenylethylene 82 to furnish a mixture of dioxane 104 and open-ring 105 (Scheme 11). ${ }^{116,}{ }_{118}$ This ratio is dependent of the alkyl group involved at position 3. Having no substituent at this position, such as with compound 106, gave rise to double addition of the olefin. The transformation drove to the formation of compounds $\mathbf{1 0 8}$ and $\mathbf{1 0 9}$, depending the carbonyl group involved in the tricyclic structure (Scheme 11).119 Other heterocycles such as hydroxybenzoquinone $\mathbf{1 1 0}$ led to similar reaction product $\mathbf{1 1 1}$ (Scheme 11). ${ }^{120}$ Tetronic acid 112, because of its prominent enol form, was also a very convenient substrate for such a transformation and 1,2-dioxanol 113 was isolated with an excellent yield (Scheme 14).121<smiles>CCCc1c(O)c2ccccc2[nH]c1=O</smiles>

103

-<smiles>Cn1c(=O)cc(O)c2ccccc21</smiles>

106<smiles>C=C(c1ccccc1)c1cccs1</smiles>
1072 eq.<smiles>C=C(CC(=O)OCC(=O)OCc1ccccc1)c1ccccc1</smiles>

82<smiles>CCCC12CC(c3ccccc3)(c3ccccc3)OOC1(O)c1ccccc1NC2=O</smiles>
6 examples $75-96 \%$ (cumulated for both products)

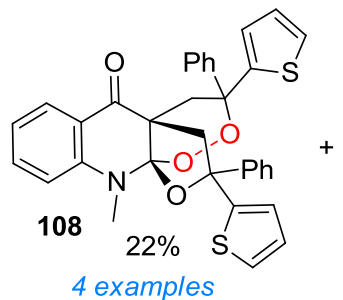

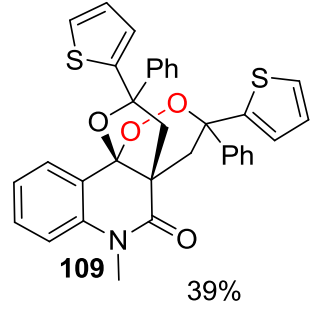<smiles>CCCC1(CC(O)(c2ccccc2)c2ccccc2)C(=O)Nc2ccccc2C1=O</smiles>

$105{ }^{\mathrm{H}} 66 \%$<smiles>CC1=C(O)C(=O)c2ccccc2C1=O</smiles>
1100<smiles>C=C(c1ccccc1)c1ccccc1</smiles>
82
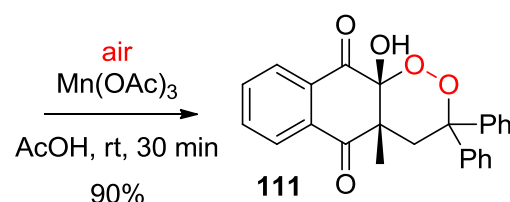

26 examples (22-90\%)

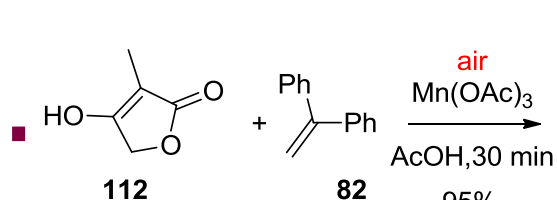

82

112

$95 \%$<smiles>CC12CC(c3ccccc3)(c3ccccc3)OOC1(O)COC2=O</smiles>
113

24 examples $35-97 \%$

\section{Scheme 14}

Intramolecular reaction was also investigated by Gademann and coworkers. ${ }^{122}$ They noticed that compound 114, an intermediate in the total synthesis of striatin A, was transformed into peroxide $\mathbf{1 1 5}$ by exposure to air and light. Manganese mediated oxidation furnished the same compound in higher yield. This compound exhibited a potent antimalarial activity (Scheme 15). 
This formal $[2+2+2]$ cycloaddition was also investigated with other metals on substrates such as compound $\mathbf{1 1 6}$ and cerium(III) chloride in presence of oxygen proved to give similar results compared to manganese (III) acetate (Scheme 15).123,124
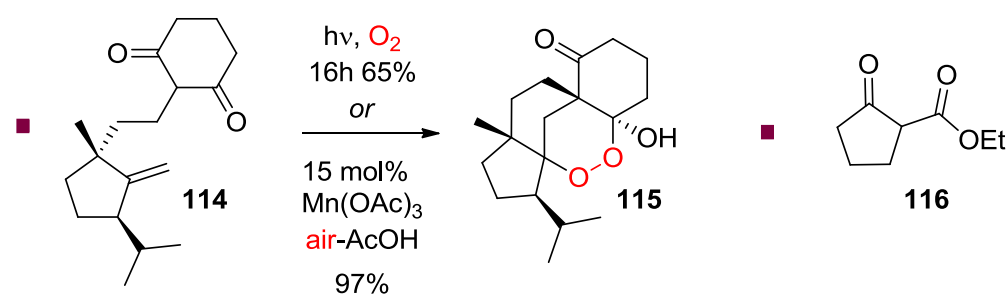

116

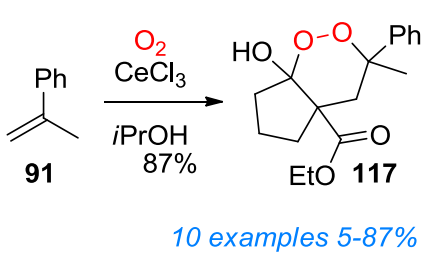

\section{Scheme 15}

This transformation was also investigated with acetylene derivatives in place of styrene. ${ }^{125}$ Therefore, after some optimizations by using notably a large excess of manganese(III) acetate, Nishino and coworkers found that acetylacetonate 118 and phenyl acetylide $\mathbf{1 1 9}$ drove to the formation of 1,2-dioxolanol $\mathbf{1 2 0}$ with modest yield. Similarly to reaction with styrene derivatives, free radical species from acetylacetonate $\mathbf{1 1 8}$ is probably adding on the triple bond, affording vinyl radical species 121. Oxygen then reacts with it and the corresponding peroxyradical species $\mathbf{1 2 0}$ is presumably reduced in situ by manganese(II) into alkoxy radical 123, which evolved favorably to alkyl radical $\mathbf{1 2 4}$ by resonance. In turn, this later reacts with another molecule of oxygen to give peroxyradical species 125, followed by the formation of compound $\mathbf{1 2 0}$ after $\mathrm{H}$ abstraction (Scheme 16).



Scheme 16

3.2.1.5 Isayama-Mukaiyama cobalt catalyzed hydroperoxysilylation

The cobalt catalyzed hydroperoxysilylation was originally developed by Isayama and Mukaiyama, ${ }^{126,127}$ and is probably the most popular method to prepare 
hydroperoxides from complex molecules since the reaction is highly chemoselective and regioselective. Indeed, the reaction usually takes place on the most electron rich olefin with formation of a radical species on the most substituted position of the reacting double bond. The mechanism was more recently better studied by Nojima, ${ }^{128}$ where he figured out that cobalt superoxide species, formed from oxygen and Co(II) salts are initiating the reaction with the help of $\mathrm{Et}_{3} \mathrm{SiH}$ to form cobalt(III) hydride species. As depicted in Scheme 16, a hydrometallation process takes then place during the hydroperoxysilylation of compound $\mathbf{1 2 6}$, and surprisingly the metal is reacting predominantly on the most substituted position (Markovnikov's rule). Cobalt(III)-carbon bond in $\mathbf{1 2 9}$ is then easily dissociating, generating alkyl radical species 130 on one side and a cobalt(II) salt on the other side. The regiochemistry appears to be guided by stabilization of this radical species rather than steric hindrance. Alkyl radical species $\mathbf{1 3 0}$ can react then with triplet oxygen to form hydroperoxy radical 131, which can be reduced with Co(II) species in order to make new superoxo-cobalt species 132, which can propagate again the process by reaction with $\mathrm{Et}_{3} \mathrm{SiH}$, in order to form a cobalt(III) hydride species (Scheme 17).

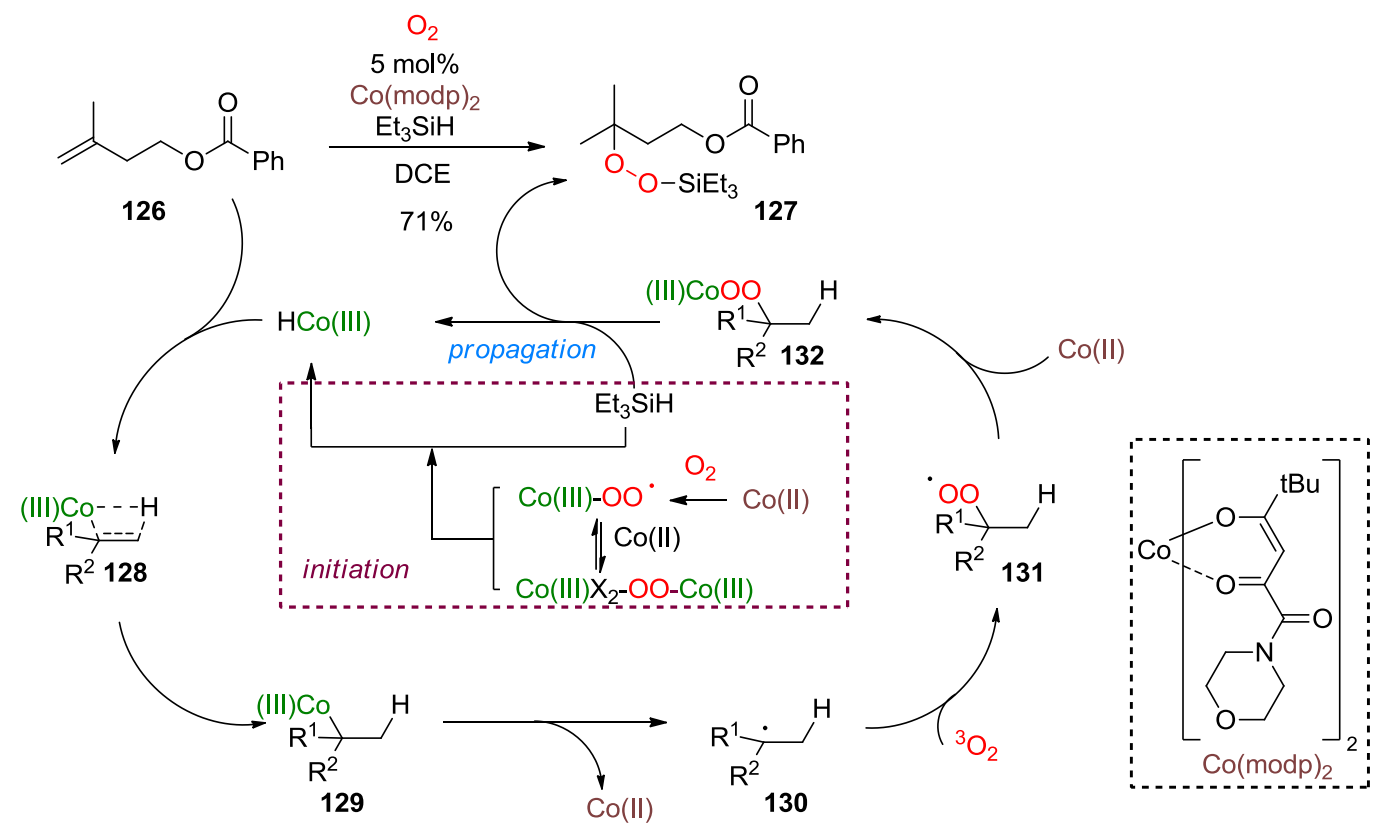

\section{Scheme 17}

Although the reaction usually does not provide an endoperoxide directly, since the hydroperoxide needs a further cyclization by different methods which would be discussed further (see paragraph 3.2.2.1, 3.3, and 3.5.1), Isayama-Mukaiyama cobalt catalyzed hydroperoxysilylation can provide directly a 1,2-dioxane or a 1,2-dioxolane depending the circumstances and the structure of the substrate. Indeed, some 1,4 or 
1,5-diene can lead directly to the formation of these endoperoxides through a radical chain reaction. A first example was described by Nojima and coworkers for the synthesis of analogs of Yingzhaosu A. ${ }^{129}$ A first hydroperoxidation from limonene with some cobalt hydride complex and oxygen was followed by an internal addition of hydroperoxy radical 133 onto the double bond at $\gamma$-position, which led to 1,2-dioxane 134. Further trapping of molecular oxygen, followed by silylation (and in a second step its desilylation with acidic $\mathrm{MeOH}$ ), drove to dioxane 135. The transformation was however modest in term of yield and led also to the formation of expected byproducts 136 and 137, coming from individual hydroperoxidation processes. Also, the reaction was applied to other limonene derivatives such as enoate $\mathbf{1 3 8}$, which gave a similar result. The major comment here is about the in situ reduction of the hydroperoxyl function in hydroxyl group with this substrate (Scheme 18).

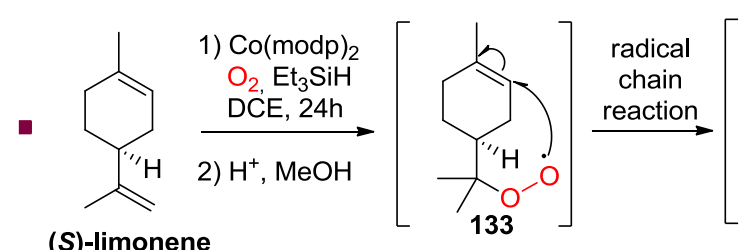

(S)-limonene
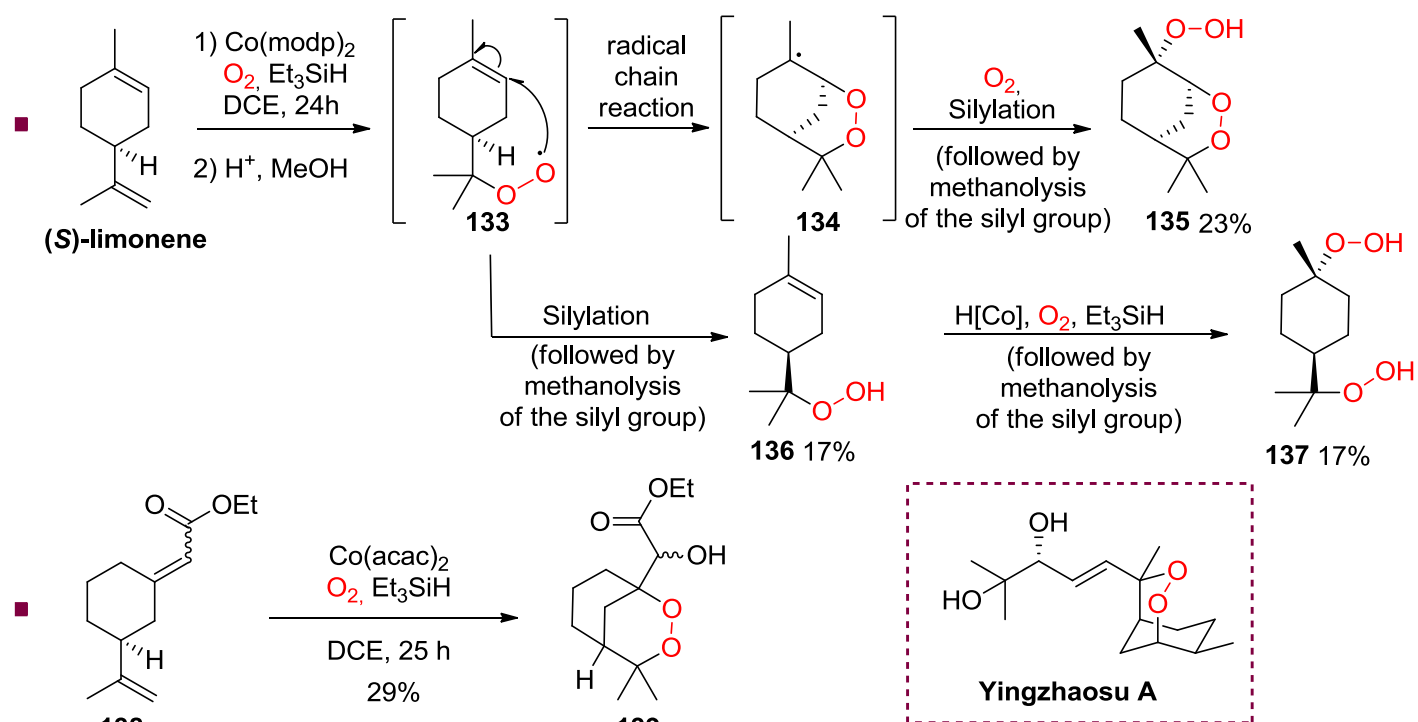

138
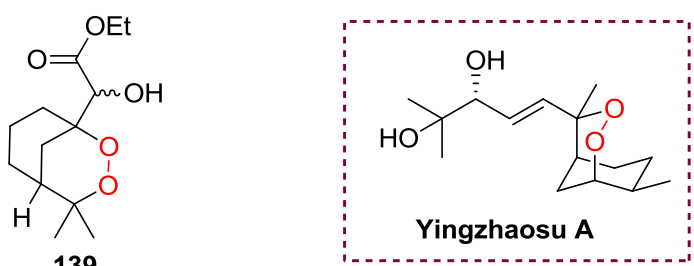

\section{Scheme 18}

The reaction was later more studied by Nojima on acyclic systems. ${ }^{130,131}$ Some specific examples with 1,4-dienes such as with substrate 140 drove to expected 1,2dioxane ring $\mathbf{1 4 1}$ with $64 \%$ yield, while some 1,3-diene systems such as compound 142 could afford 1,2-dioxolane structure 143 under similar reaction conditions. (Scheme 19). 

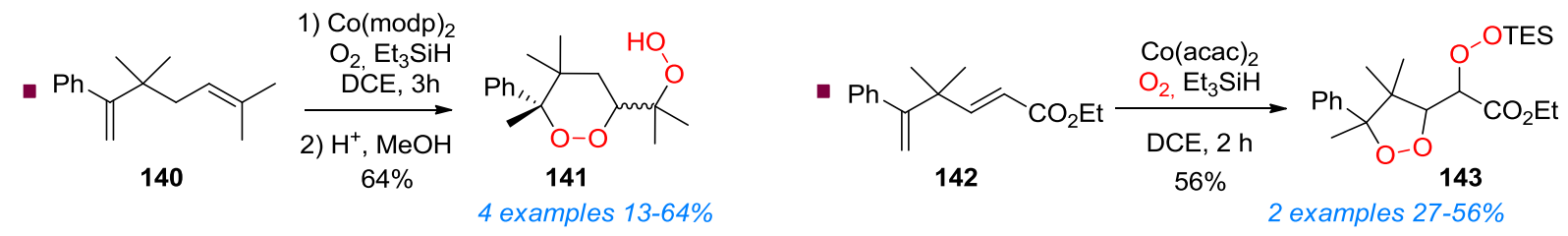

\section{Scheme 19}

This reaction was more recently applied as a key step in the total synthesis of many endoperoxides of chamigrane family.132 Thus, spirocompound 144 was transformed in a mixture of diastereomers $\mathbf{1 4 5} \mathbf{a}$ and $\mathbf{1 4 5} \mathbf{b}$ by using the IsayamaMukaiyama cobalt catalyzed hydroperoxysilylation. The mixture of diastereomers was treated with acidic methanol to cleave the silyl ether, followed by reduction of the hydroperoxide with dimethylsulfide to give steperoxide $\mathrm{C}$ (if considering appropriated diastereomer $\mathbf{1 4 5}$ b). Further oxidation of the alcohols in ketone drove to talaperoxide $\mathrm{C}$. Other natural endoperoxides of the chamigrane family have been obtained from these compounds (Scheme 20).

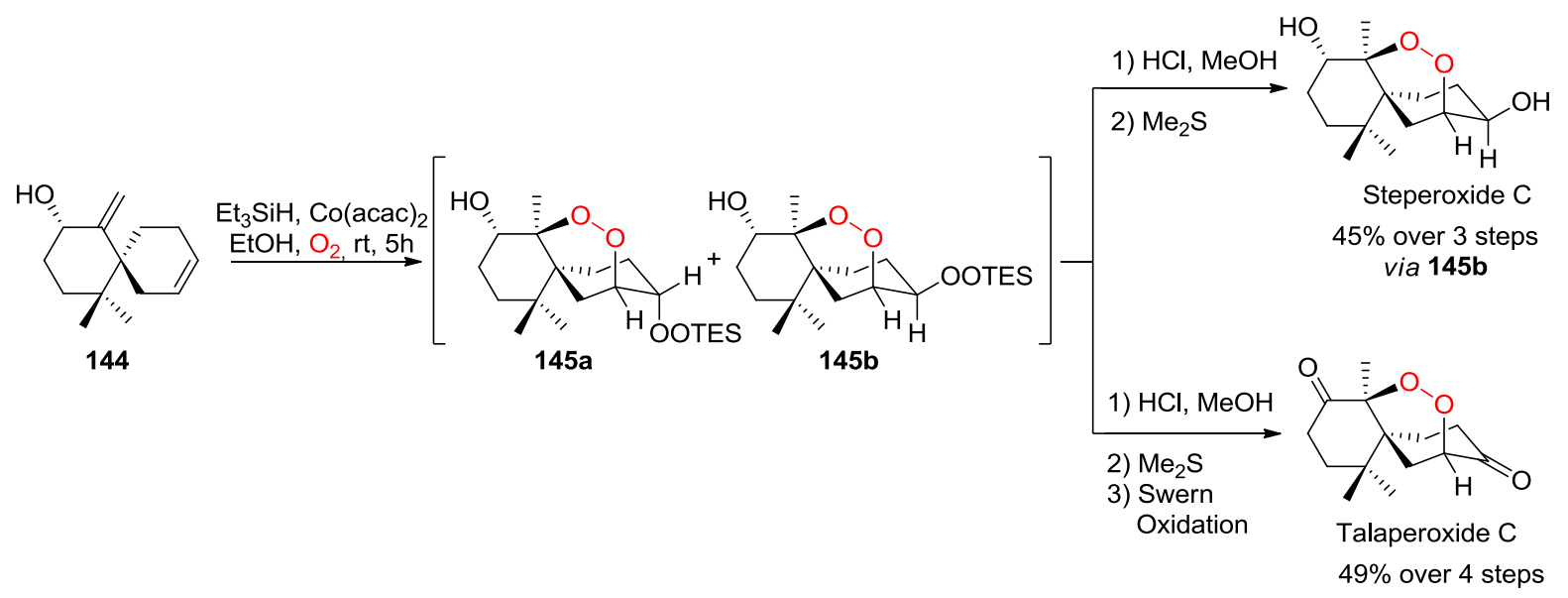

\section{Scheme 20}

This radical chain process was also used in a recent work towards the total synthesis of nortribolide. Contrary to the last example (see Scheme 20), the formation of the endoperoxide is just a fulcrum for the synthesis of a specific triol system via the peroxide bond reduction with zinc. ${ }^{33}$ Thus, intermediate 146 was treated under Isayama-Mukaiyama reaction conditions and led to a mixture of compounds 147 and 148 with 10 and 16\% yields, respectively, after further optimizations. The yield was not very high but allow to access to a highly functionalized structure with a high chemoselectivity and stereoselectivity. It is also interesting to note that no peroxysilyl ether was isolated, but directly the alcohol function. Such as with compound $\mathbf{1 3 8}$ (Scheme 18), the electron withdrawing group 
seems to have an effect on the reducibility of the peroxyradical intermediate. Then, because of the low yield, the authors wanted to test their procedure on other simpler systems such as with eudesmane sesquiterpene $\mathbf{1 5 0}$ in order to give triol 152 through reduction of 151. Due to the presence of only one remaining olefin in the system, the overall yield after reduction was more satisfying (50\% vs 16\%) (Scheme 21).

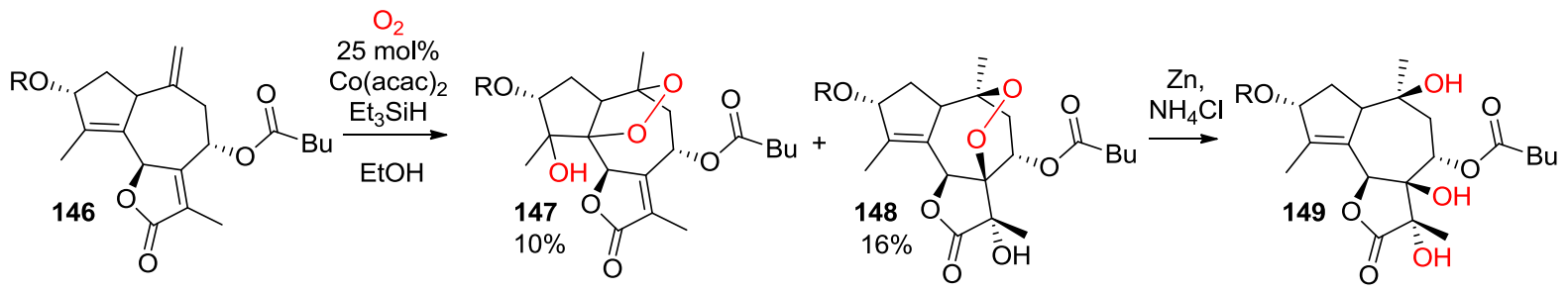

$R=$ TBDPS
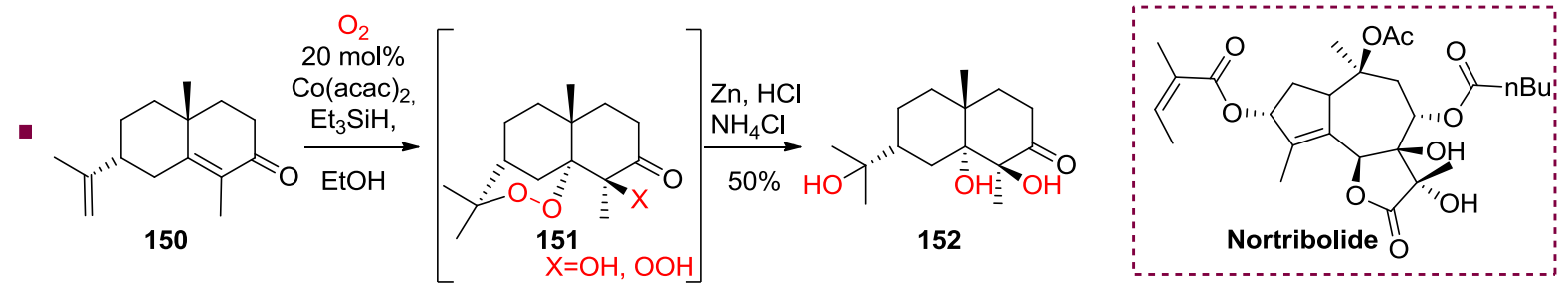

Scheme 21

We saw that 1,4 and 1,3-dienes could be appropriate substrates to make endoperoxides in the Isayama-Mukaiyama cobalt catalyzed hydroperoxy-silylation, but strained cycles could be also a good alternative to prepare such compounds (see also paragraphs 3.2.1.3 and 3.2.1.5), although examples are more limited. For example, Nojima showed that vinyl cyclopropane $\mathbf{1 5 3}$ could be opened through the formation of radical species $\mathbf{1 5 4}$ at the adjacent position of the strain ring, followed by formation of 155, which led to trapping of oxygen and ring closing onto the olefin. Further reaction of radical $\mathbf{1 5 6}$ with oxygen followed by silylation drove to 1,2dioxolane 158 (Scheme 22). ${ }^{128}$

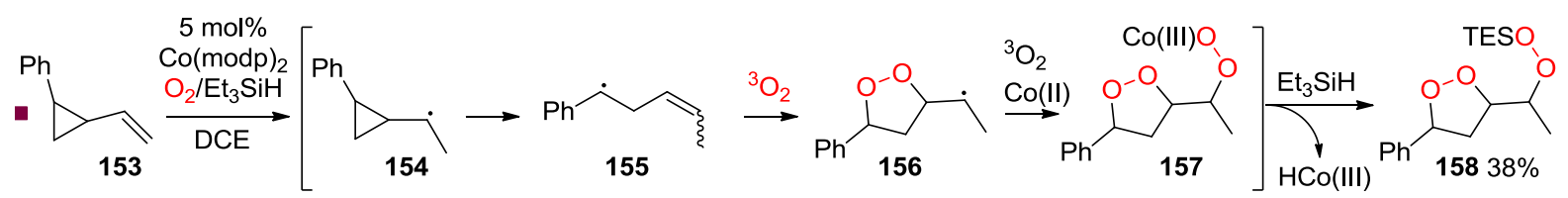

Scheme 22

Similarly, Enders used this process as a keystep in the final stage of the total synthesis of gracilioether A. Notably, the ring-opening of the cyclobutane contained in 159 led to the formation of a 1,2-dioxane ring corresponding to gracilioether A, with $39 \%$ yield and its epimer with $16 \%$ yield. Authors obtained directly the alcohols derivatives rather than the usual triethylsilylperoxy ethers such as with compounds 
138 and 146 (Scheme 18 and 21), but unfortunately this phenomenon is not clearly described by the authors (Scheme 23).134

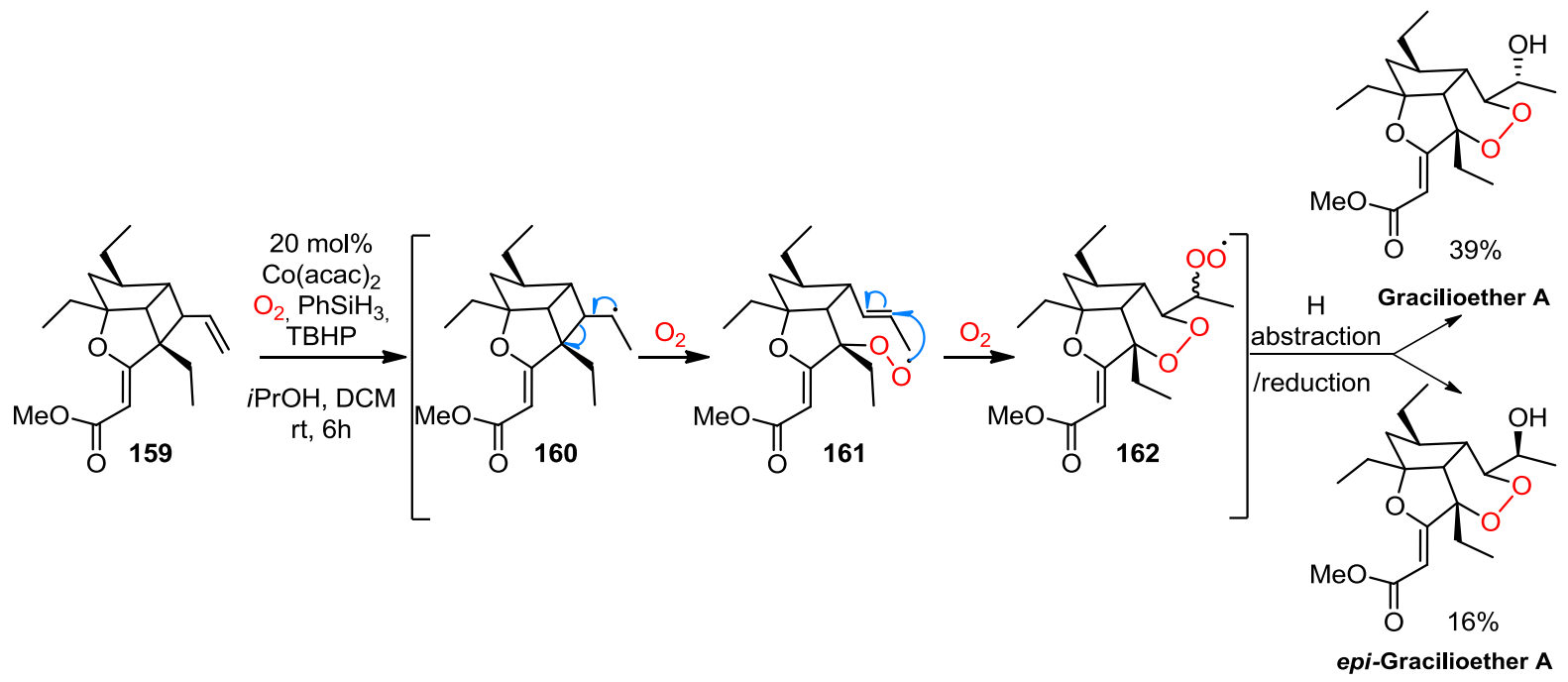

Scheme 23

\subsubsection{Thiyl and selenyl radical-mediated domino reactions}

Addition of radical thiyls to olefins under oxygen atmosphere is an old reaction discovered by Kharasch and coworkers in 1950 and called TOCO reaction (ThioOlefin Co-Oxidation). ${ }^{135}$ This reaction consists, such as in the example depicted in Scheme 23, in the transformation of $n$-propylthiol, styrene and oxygen into sulfoxyalcohol 161. Mechanistically, thiyl radical 164 (formed by oxygen or any other radical initiator) is adding onto styrene, giving free radical species $\mathbf{1 6 5}$, and this step is followed by addition of triplet oxygen and internal oxidation of the sulfide by the peroxy species to give $\mathbf{1 6 7}$ and then 163. (Scheme 24)

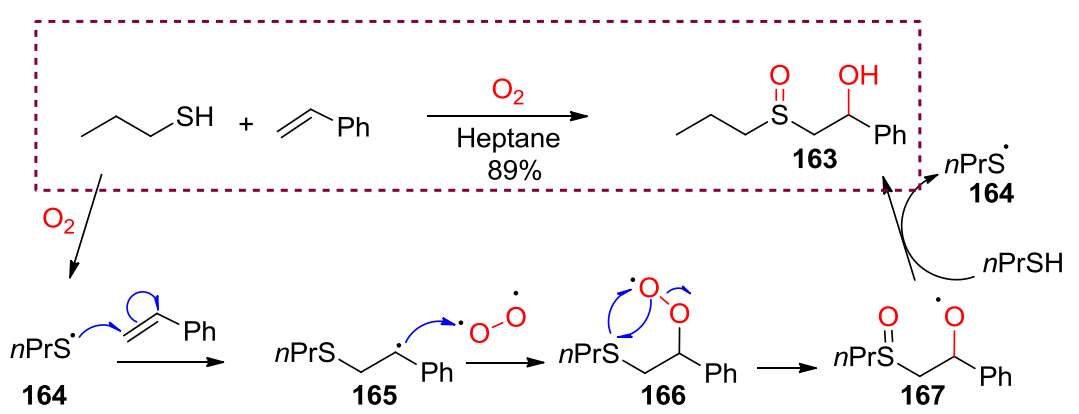

\section{Scheme 24}

The application of this reaction many years later by Beckwith and coworkers to dienes ${ }^{136}$ and trienes ${ }^{137,138}$ allowed the preparations of 1,2-dioxolanes when two double bonds were spaced by one methylene segment. Thus, authors could transformed triene 168 into 1,2-dioxolane 169 through the addition of a thiyl radical 
to terminal olefin and oxygen trapping of allyl radical $\mathbf{1 7 0}$, which can then cyclize in turn onto the last olefin in order to perform a free radical chain reaction with another molecule of oxygen. In contrast to the example of Kharasch with a substrate containing only one olefin (Scheme 24), the reaction here does not produce sulfoxide through the reduction of the peroxyradical, keeping the sulfide function. Since the hydroperoxides are more reactive, its selective reduction with $\mathrm{PPh}_{3}$ guided 169 to alcohol 172 with $49 \%$ yield over 2 steps. It is also noteworthy that the reaction was surprisingly diastereoselective since only one isomer was observed (Scheme 25).

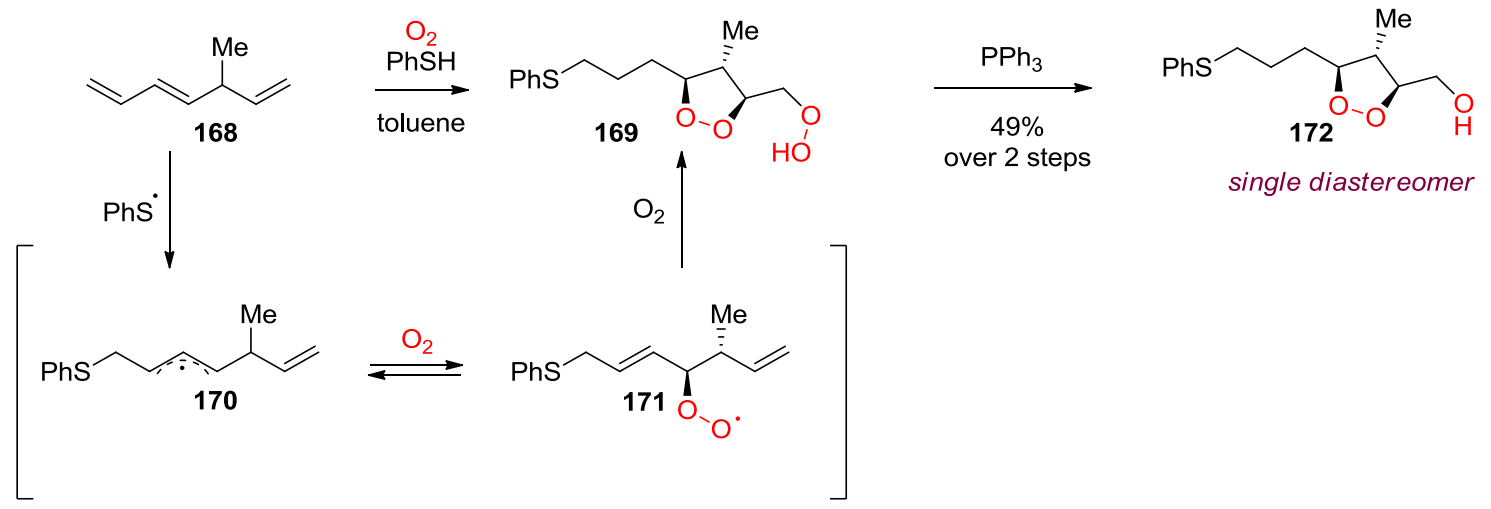

\section{Scheme 25}

About twenty years later, Bachi and coworkers became interested in the total synthesis of Yingzhaosu A59 and several of its synthetic analogues. They found that the application of TOCO reaction on limonene and limonene derivatives would be an elegant pathway to this antimalarial substance, which contains a 1,2-dioxane ring into a bicyclic structure. ${ }^{139}$ Compared to Beckwith and coworkers (Scheme 25), they finally optimized the conditions using a radical initiator such as AIBN or dibenzoyl peroxide in conjunction with light and acetonitrile as solvent. ${ }^{140,142}$ Thus, limonene was for example transformed into a mixture of endoperoxides $174 \mathbf{a}$ and $\mathbf{1 7 5} \mathbf{b}$, corresponding to the formation of four new bonds in only one step! The sulfides were oxidized into sulfoxides 176a and 177a and then transformed into aldehyde through a Pummerer rearrangement. This sequence allowed the access to Yingzhaosu A after Mukaiyama aldol reaction and further transformations, but also to many other analogues of this molecule such as $\mathbf{1 8 1}$ or $\mathbf{1 8 2}$. ${ }^{143,144}$ Moreover, the synthesis of other analogues from limonene derivatives $\mathbf{1 7 3} \mathbf{b}-\mathbf{e}$ drove to the preparation of many other products such as $\mathbf{1 7 8}$ or compounds 179 or $\mathbf{1 8 0}$ through some Wittig olefination. These two last compounds showed a remarkable anti-malarial activity. ${ }^{144}$ (Scheme 26) 


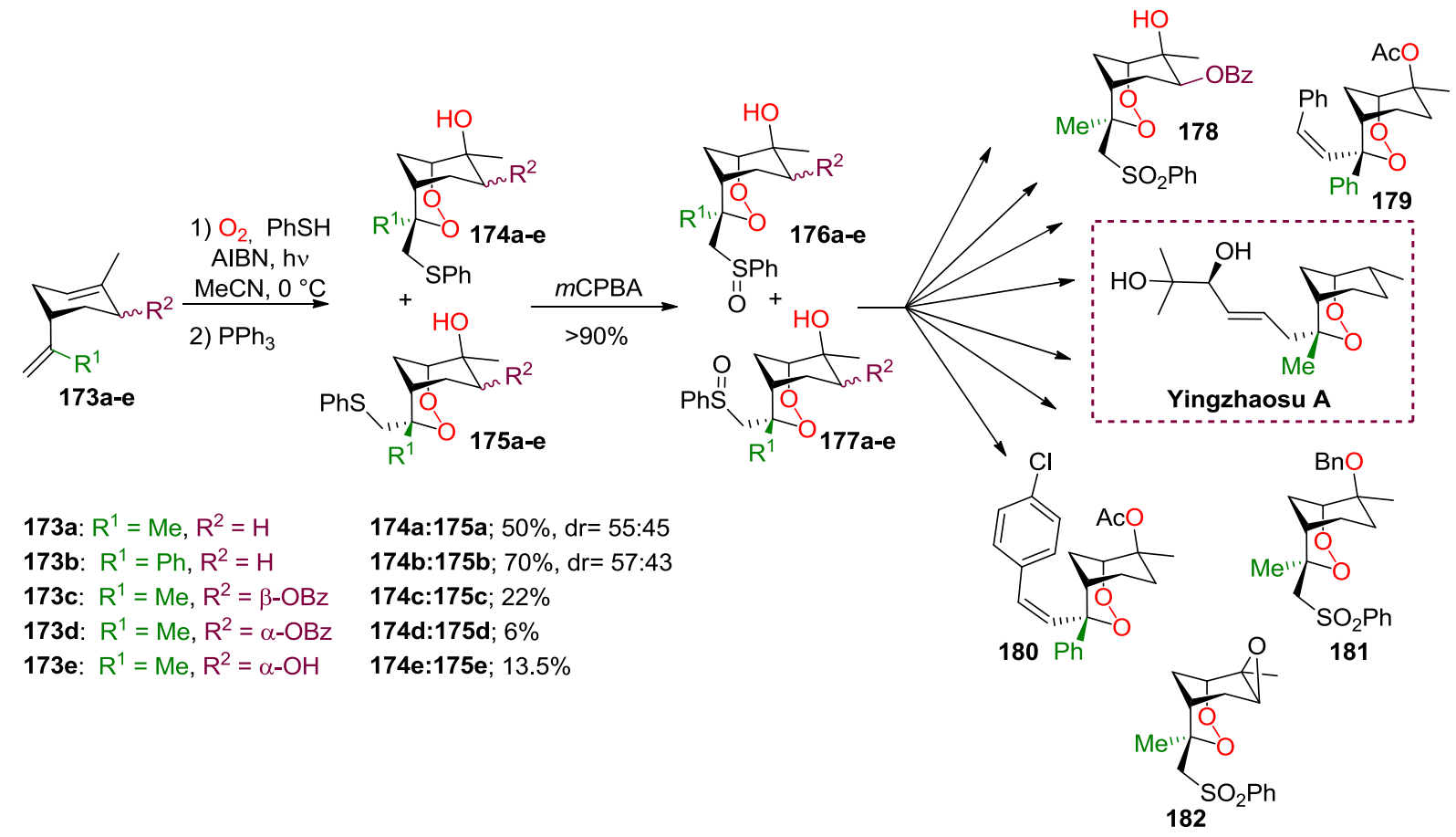

\section{Scheme 26}

In parallel to Beckwith's work with dienes or trienes (vide supra), Feldman and coworkers developed in 1986 a convenient approach for the formation of 1,2dioxolanes $\mathbf{1 8 4}$ from 1-vinylcyclopropanes $\mathbf{1 8 3}$ bearing phenyl, vinyl or ester substituents at C-2 position of the cyclopropyl ring or alkyl groups at C-1 of the vinyl moiety. Like many radical reactions involving a cyclopropane, this method is based on the $\beta$-elimination of cyclopropylmethyl radicals, followed by addition of oxygen triplet. ${ }^{145,146}$ In contrast to the classical TOCO reaction, the newly formed thiyl or selenyl substituent undergoes a final elimination. Indeed, the addition of phenylselenide (or thiyl) radical to alkene $\mathbf{1 8 3}$ drives to cyclopropylmethyl radical 185, which rearranges into homoallyl radical 186 through a $\beta$-fragmentation. This alkyl radical is then trapped by $\mathrm{O}_{2}$, providing peroxy radical $\mathbf{1 8 7}$, which after cyclization and $\beta$-elimination of phenylselenyl radical, affords desired dioxolane $\mathbf{1 8 4}$. Thus, numerous examples of this reaction have been reported giving yields ranging between $41 \%$ and $88 \%$. The application of this process to dicyclopropyl compound 189 afforded two contiguous dioxolanes in a mixture of diasteromers 1909 and 190b, while tricyclopropyl substrate 191 drove to product 192 in $38 \%$ yield, with formation of three consecutive 1,2-dioxolane rings, i.e. six new carbon-oxygen bonds in one step! Sulfur showed to be especially effective in this last transformation compared to selenium, which seems to be more suited and selective towards the construction of a single dioxolane ring (Scheme 27). 


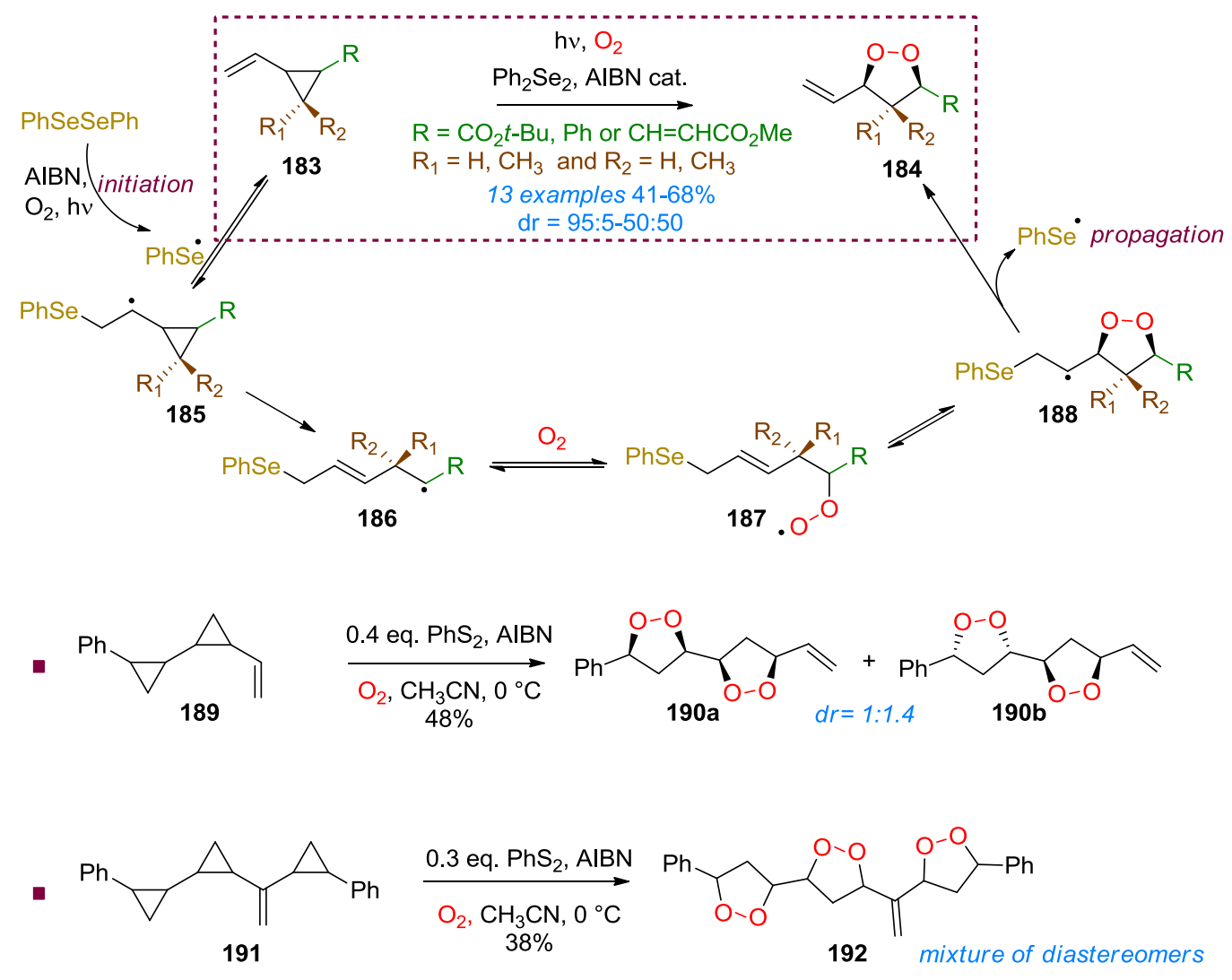

\section{Scheme 27}

Feldman and coworkers were also interested to show that 1,2-dioxolanes could lead to the formation of 1,3-diol. Therefore, they also applied their methodology to the total synthesis of (+/-)-yashabushitriol from vinyl cyclopropane 193. The thiyl radical gave a better $E$ selectivity for the formation of compound 194, compared to the selenide radical, although this latter gives a slightly higher yield for the transformation into 1,2-dioxolane. The reductive opening of 194 into diol derivative 195 drove to the formation of yashabushitriol after 3 more steps (Scheme 28). ${ }^{147}$

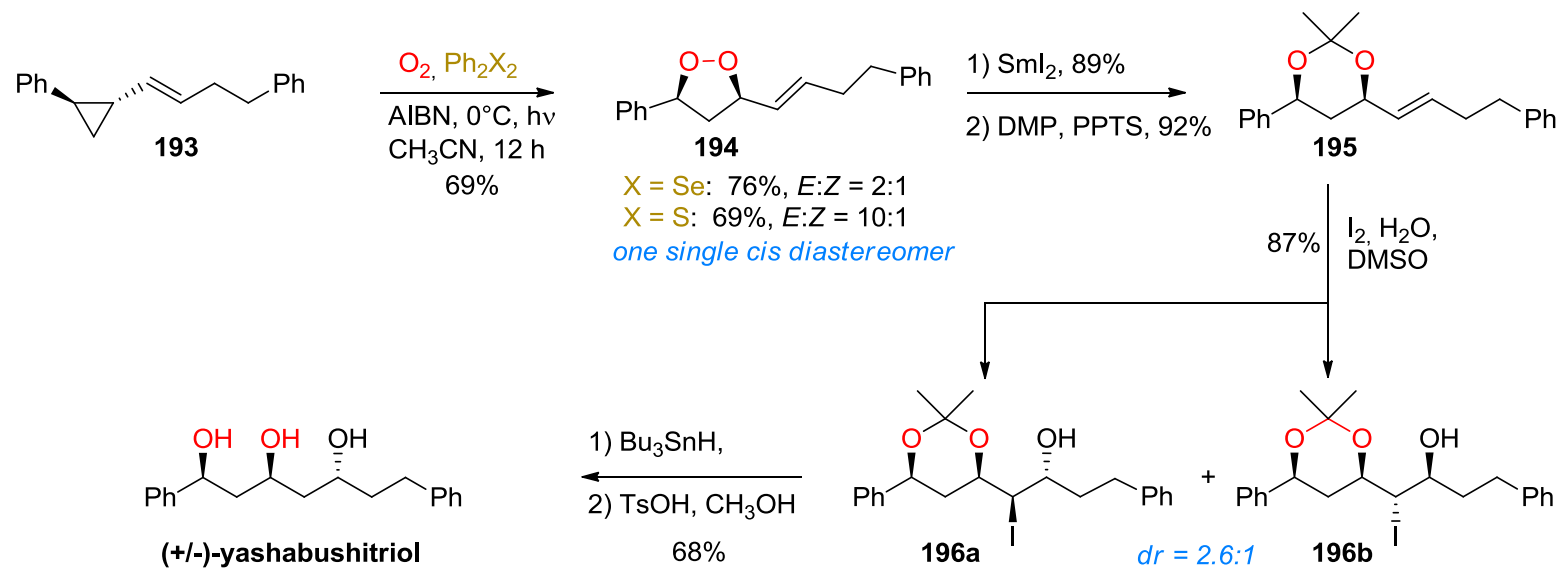

Scheme 28 
The Feldman's protocol was also applied to the total synthesis of natural products some years later by Wong and coworkers. In a first work, they accomplished the total synthesis of Plakortide E, by applying Feldman's protocol to lactone 197.148 Because of the cyclic nature of $\mathbf{1 9 7}$, only one diasteromer could be obtain. Further reduction of the lactone and the olefin with diimide gave meso-diol 199. A mono protection with a silyl group gave alcohol 200 as a racemate. A kinetic resolution with vinyl acetate and Lipase PS from Bukhoderia cepaci in hexane gave unreacted alcohol (+)-201 or acetate (-)-202 with >99\%ee for both depending the reaction time, respectively $29 \mathrm{~h}$ or $3 \mathrm{~h}$. Further functionalization of (+)-201 or (-)-202 over 11 steps afforded the different enantiomers and diastereomers of Plakortide E. A NMR spectroscopy comparison, as well as a study of the optical rotation sign of the synthetic samples and the natural product confirmed absolute stereochemistry of the 3 sterogenic centers of plakortide E (Scheme 29).

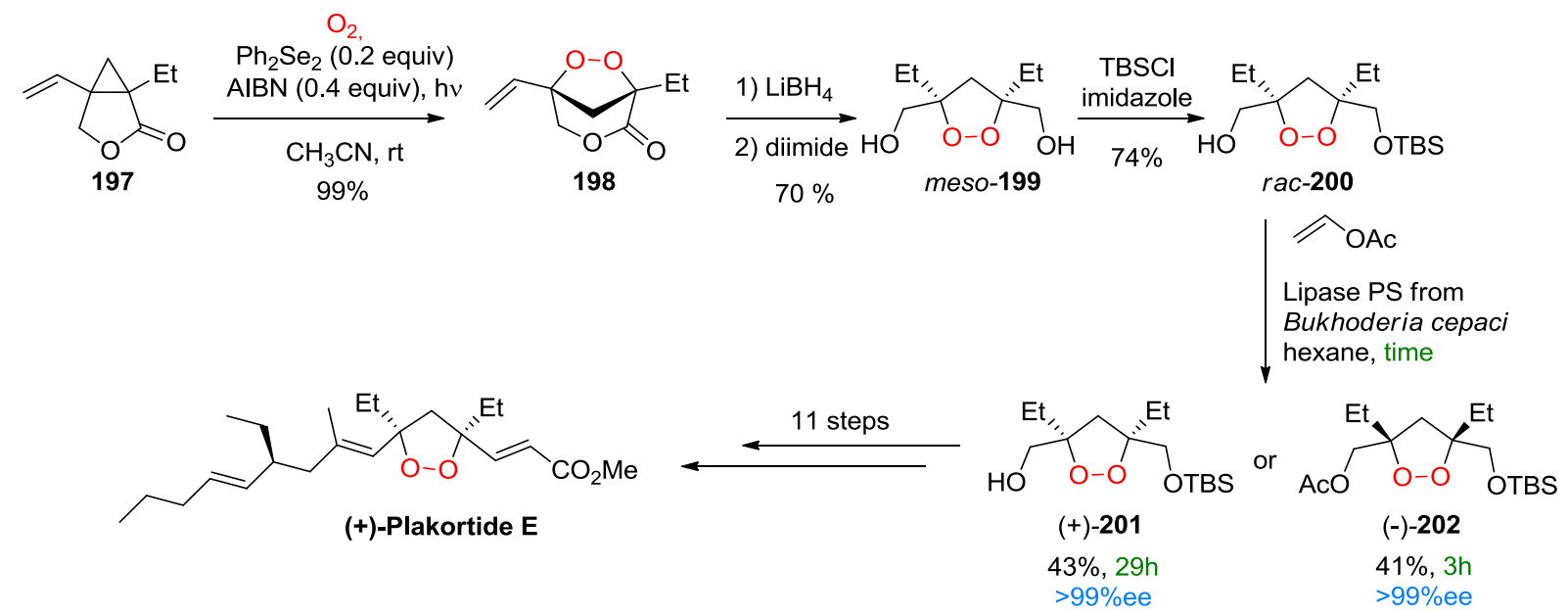

Scheme 29

Wong and coworkers were also interested in other natural endoperoxides, such as epi-plakinic acid F. For this natural substance, authors were interested to use a diastereoselective Feldman's reaction using a chiral oxazolidinone auxiliary. ${ }^{22}$ After a screening of different conditions, solvent and chiral auxiliaries, the oxazolidinone derived from valine appeared to be a better chiral inductor, and the use of Sc(OTf $)_{3}$, among all other Lewis acids tested as additives, gave the best result in term of diatereoselectivity. Indeed, trans-isomer 205b was isolated in $72 \%$ yield from a mixture of three isomers (90\% total yield containing $80 \%$ of isomer $\mathbf{2 0 5} \mathbf{b}$ ). Scandium triflate probably forces the two carbonyl groups to be oriented on the same side due to chelation, such as on intermediate $\mathbf{2 0 6}$, improving the rigidity of the chelate and by consequence the stereoselectivity of the alkyl radical addition to the 
oxygen at the top face of intermediate $\mathbf{2 0 6}$ (80\% selectivity). Addition of peroxy radical to the olefin depends more on the conformation of the olefin, but it seems that it adopts the one described in $\mathbf{2 0 6}$ since it drives to the major observed stereochemistry (89\%). After 12 steps from 205b including a carbonyl group homologation and some palladium catalyzed cross coupling reactions to build the fatty chain, the authors could access an enantiopure synthetic sample of epi-plakinic acid F (Scheme 30).

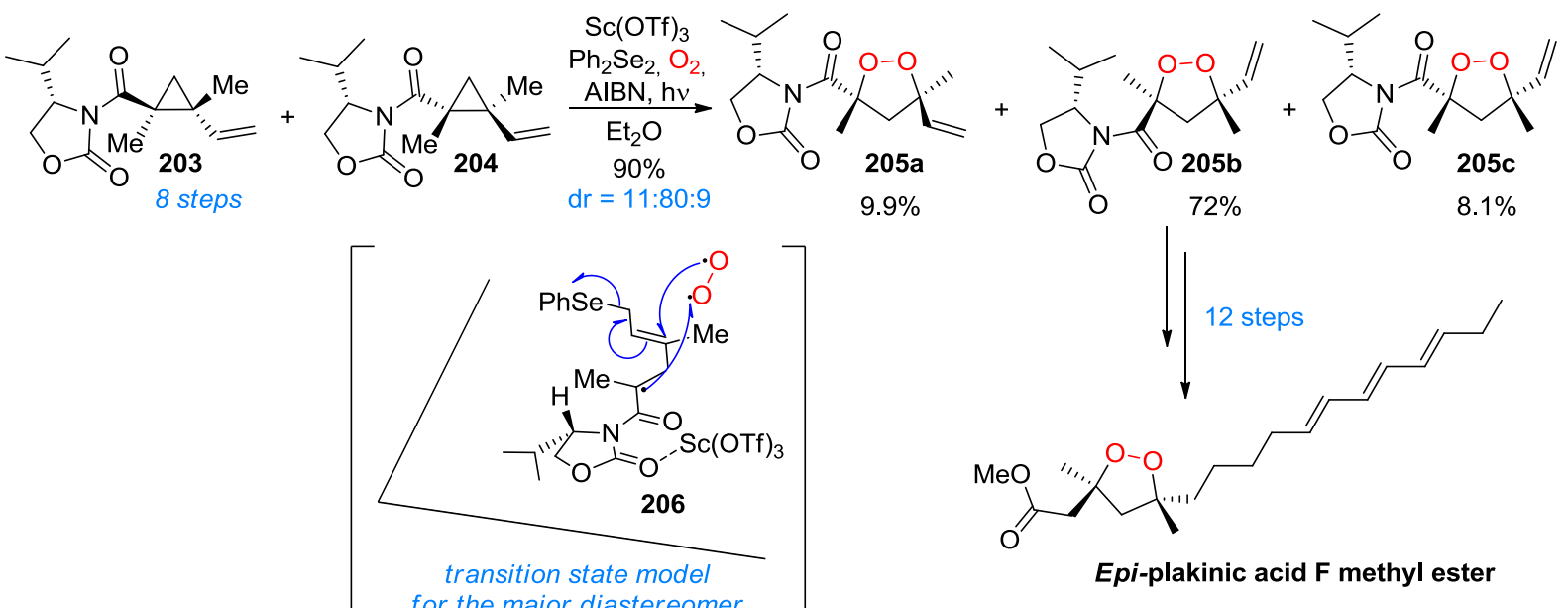

Scheme 30

3.2.1.7 Organozinc oxidation through an electrophilic activation of a cyclopropane

Organometallics are known to react with oxygen to produce hydroperoxides or more generally hydroxyl compounds, after an in situ reduction. In particular, organoborons ${ }^{149}$ and organozinc150 compounds were reported to produce more chemoselectively hydroperoxides. Although the mechanism of this oxidation is not completely clear, the transformation seems to be a free radical reaction. Recently, López and Vicente reported an original transformation to obtain some furanyldioxolanes. ${ }^{151}$ Indeed, from acetylacetonate 206, ynal 207, and olefin 82 in presence of $\mathrm{ZnCl}_{2}$ (2 mol\%) in solvent free conditions, they could obtained acetylfuran $\mathbf{2 0 8}$, substituted with a cyclopropyl ring. This multi-component reaction works first through the condensation of $\mathbf{2 0 6}$ onto $\mathbf{2 0} 7$ to obtain intermediate 209. The carbonyl group is then cyclizing onto the alkyne giving 210, via an activation of the zinc catalyst. Organozinc species is probably giving back a negative charge by conjugation to the oxocarbenium species, driving to furanyl structure $2 \mathbf{1 1}$ containing a zinc carbenoid (proposed intermediate). Taking advantage of this carbenoid, a cyclopropanation takes place with an olefin such as $\mathbf{8 2}$ to afford cyclopropane $\mathbf{2 0 8}$. 
Compound 208 could further react with a larger amount of $\mathrm{ZnCl}_{2}$ under oxygen atmosphere to produce 1,2-dioxolane 212. Reasonably, $\mathrm{ZnCl}_{2}$ can activate the cyclopropyl ring, which exhibits a $\pi$-character and the oxygen on the furan ring pushes the electron by conjugation leading to a cyclopropyl ring opening, which leads to organozinc species 213. This step is followed by insertion of oxygen in order to form probably species 214. A conjugated addition of zinc peroxide affords compounds 212. The reaction was applied to 6 examples in order to make endoperoxy structures with yields between 62 and $89 \%$, however the method is only limited to diphenylsubstituted cyclopropyl rings for the oxidation step. It is noteworthy that the reaction could be also performed from compounds 206, 207 and 208 directly in one step if the catalytic charge of $\mathrm{ZnCl}_{2}$ is increased to $50 \mathrm{~mol} \%$ directly and if the reaction is performed under oxygen atmosphere (Scheme 31).

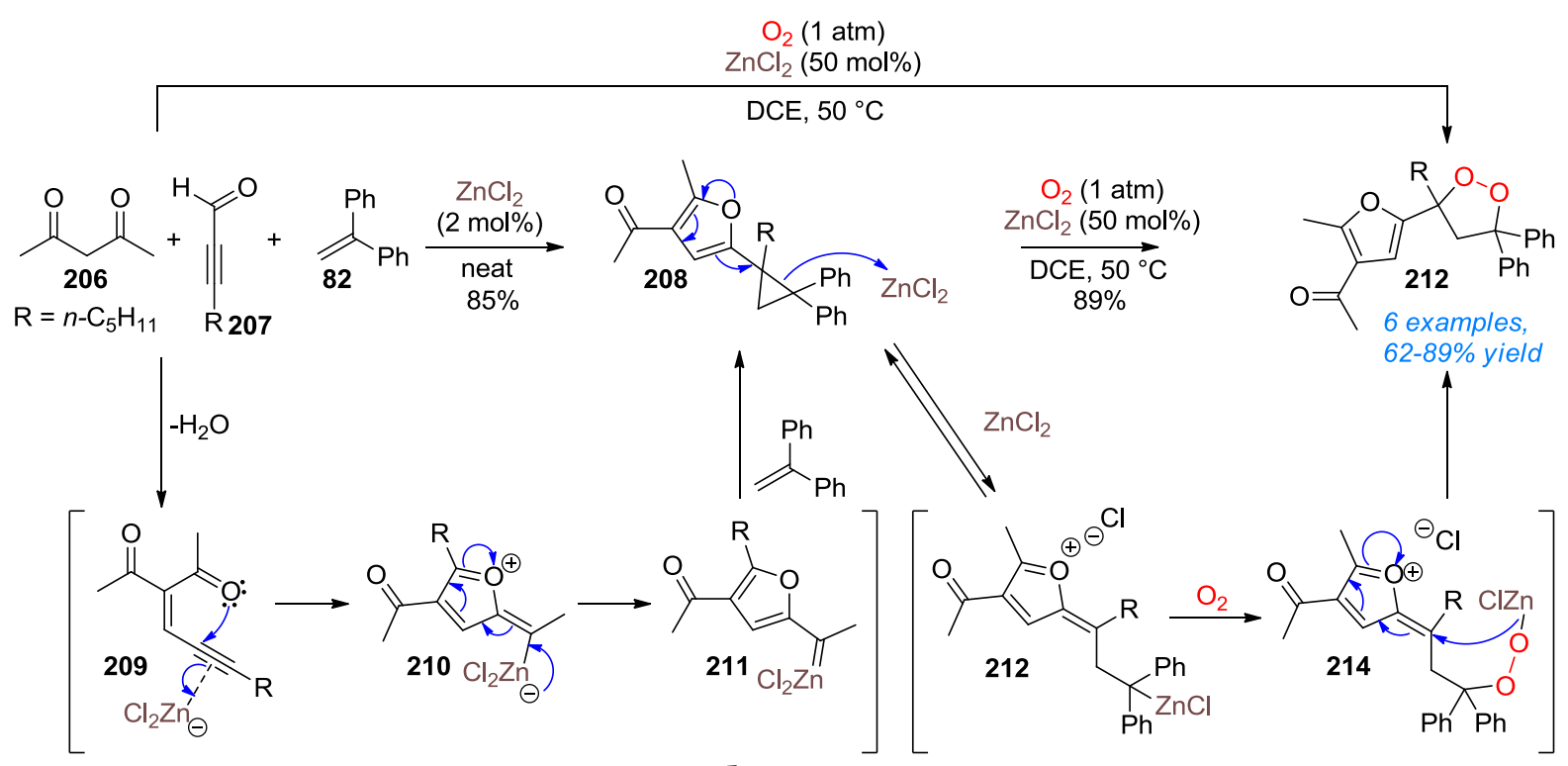

Scheme 31

\subsubsection{Radical Peroxycyclization of Hydroperoxides}

\subsubsection{Synthesis of hydroperoxides: a summary.}

We saw previously how possible is the formation of endoperoxides through a free radical chain reaction involving oxygen and allowing the formation of two new carbon-oxygen bonds in a single transformation. Another strategy to prepare 1,2dioxolanes or 1,2-dioxanes would be to start from a molecule containing a hydroperoxide function to cyclize it. The question would be then how to prepare these hydroperoxides intermediates? Since this book chapter is more focused on the formation of endoperoxides rather than hydroperoxides, and since their reaction 
classification would be impossible if we consider both formation of hydroperoxides and their further cyclization (because any combination could be performed), it is preferable to not focus on the description of the synthesis of hydroperoxides.

Nevertheless, it is a mandatory to expose concisely the different methods and strategies, which can be applied for the synthesis of hydroperoxides, in order to give to the reader an overview, although some reviews describe their preparation. ${ }^{152,153}$ Therefore, synthesis of hydroperoxides, as well as synthesis of endoperoxides (vide supra Figure 7), can be classified in three main routes:

-Free radical reactions, which involve triplet oxygen, such as the IsayamaMukaiyama cobalt catalyzed hydroperoxy-silylation of olefin;126,127 however oxidations of some organometallics such as boranes ${ }^{149}$ or organozinc reagents ${ }^{150}$ have also to be considered.

-Nucleophilic additions, which involves hydrogen peroxide and some of its derivatives; any electrophile is generally suitable to some extent: ketones and aldehydes 154 as well as epoxides ${ }^{155}$ are suitable substrates under acid catalysis, nucleophilic substitution to halides is also suitable, but generally the reaction is more appropriated on primary halide; ${ }^{156}$ however the tertiary halides or alcohols can generate a carbocation which can also react to form hydroperoxides. 157 Addition of hydrogen peroxide to an activated olefin through a peroxymercuration 158 or a peroxyhalogenation ${ }^{159}$ provides related compounds.

-Finally electrophilic reactions involving ozone or singlet oxygen; oxygen singlet provides allylic hydroperoxides through an ene-type reaction, ${ }^{160}$ whereas ozone, in presence of a protic solvent provides hemiperoxyketals (Figure 8).161 


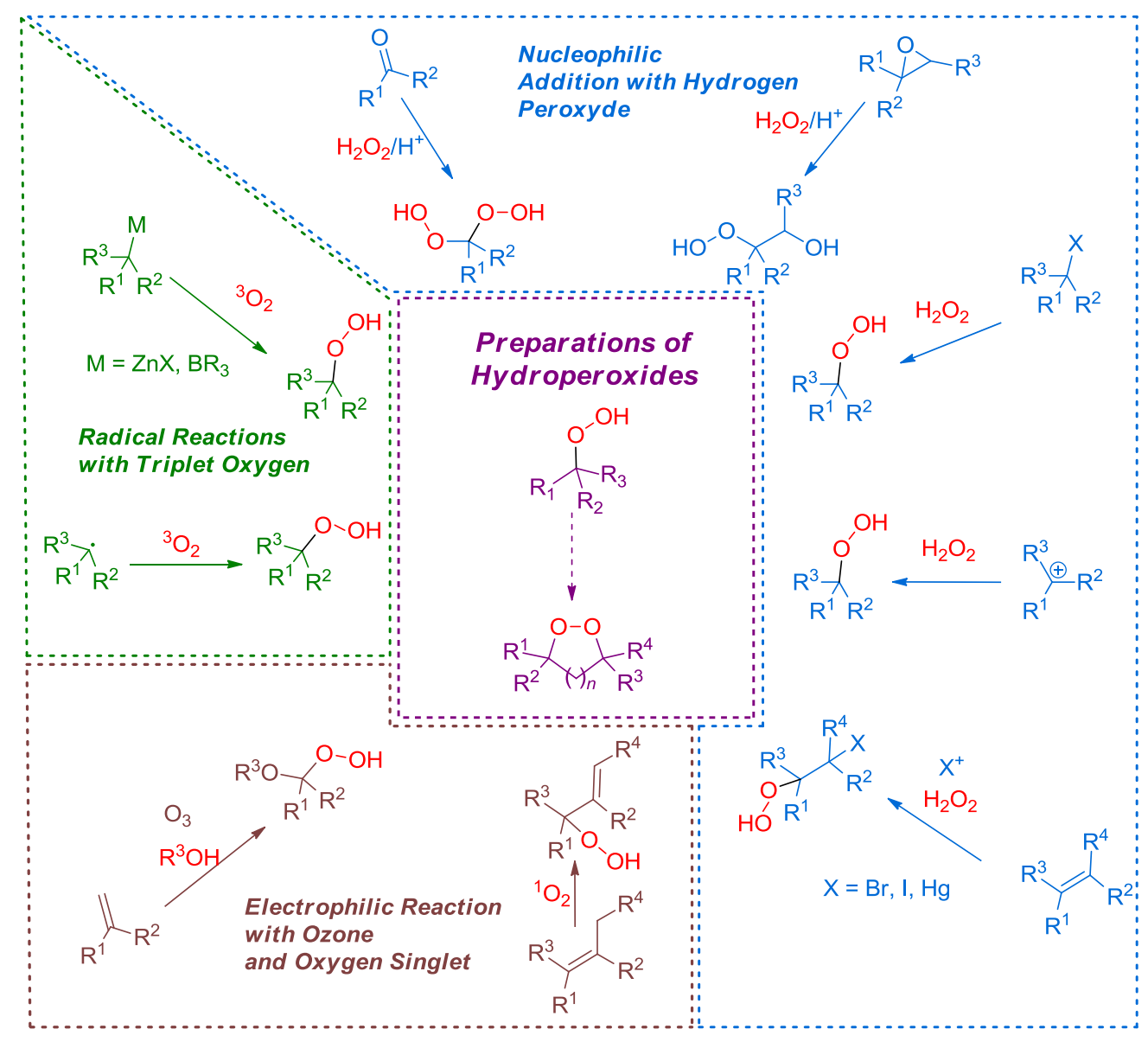

Figure 8. General strategies and pathways to build hydroperoxides.

\subsubsection{Free radical cyclization of hydroperoxides.}

There are few examples of a free radical cyclization of hydroperoxides in the literature. However an interesting example from Carless and coworkers in 1982 showed that a ene-reaction with singlet oxygen (generated in situ by photoactivation with tetraphenylporphyrine) on diene $\mathbf{2 1 5}$ afforded a mixture of hydroperoxides 215a-c in 21, 11, and 13\% yield, respectively, through an ene-reaction with singlet oxygen. Further radical mediated cyclization of hydroperoxide $\mathbf{2 1 5 c}$ in presence of triplet oxygen and a radical initiator such as di-tert-butylperoxyoxalate (DBPO) at room temperature afforded 1,2-dioxolane $\mathbf{2 1 6}$ in 20\% yield with the cis stereochemistry. The radical initiator allows the production of peroxy radical species, which can then cyclize and trap a new molecule of oxygen (Scheme 31). ${ }^{162}$

Some years later, Boukouvalas and coworkers applied this methodology to the synthesis of Yingzhaosu C (see paragraph 2.2.1). ${ }^{163}$ Thus, tertiary alcohol 218 was treated with an ethereal solution of hydrogen peroxide in presence of amberlyst to accomplish a substitution via a carbocation to form hydroperoxide 219. Treatment 
under the conditions that Carless described, with DBPO in oxygen atmosphere, afforded a mixture of 1,2-dioxanes 220 and $\mathbf{2 2 0 b}$ in a 1.7:1 ratio. Addition of tertbutyl hydroperoxide improved significantly the yield, up to $89 \%$. Chemoselective reduction of the hydroperoxide with $\mathrm{PPh}_{3}$ afforded racemic Yingzhaosu $\mathrm{C}$ and its epimer in few steps. This work allowed them to clearly reassign the relative configuration of Yingzhaosu $\mathrm{C}$ as cis, whereas natural product was initially reported as trans (Scheme 32). ${ }^{60}$
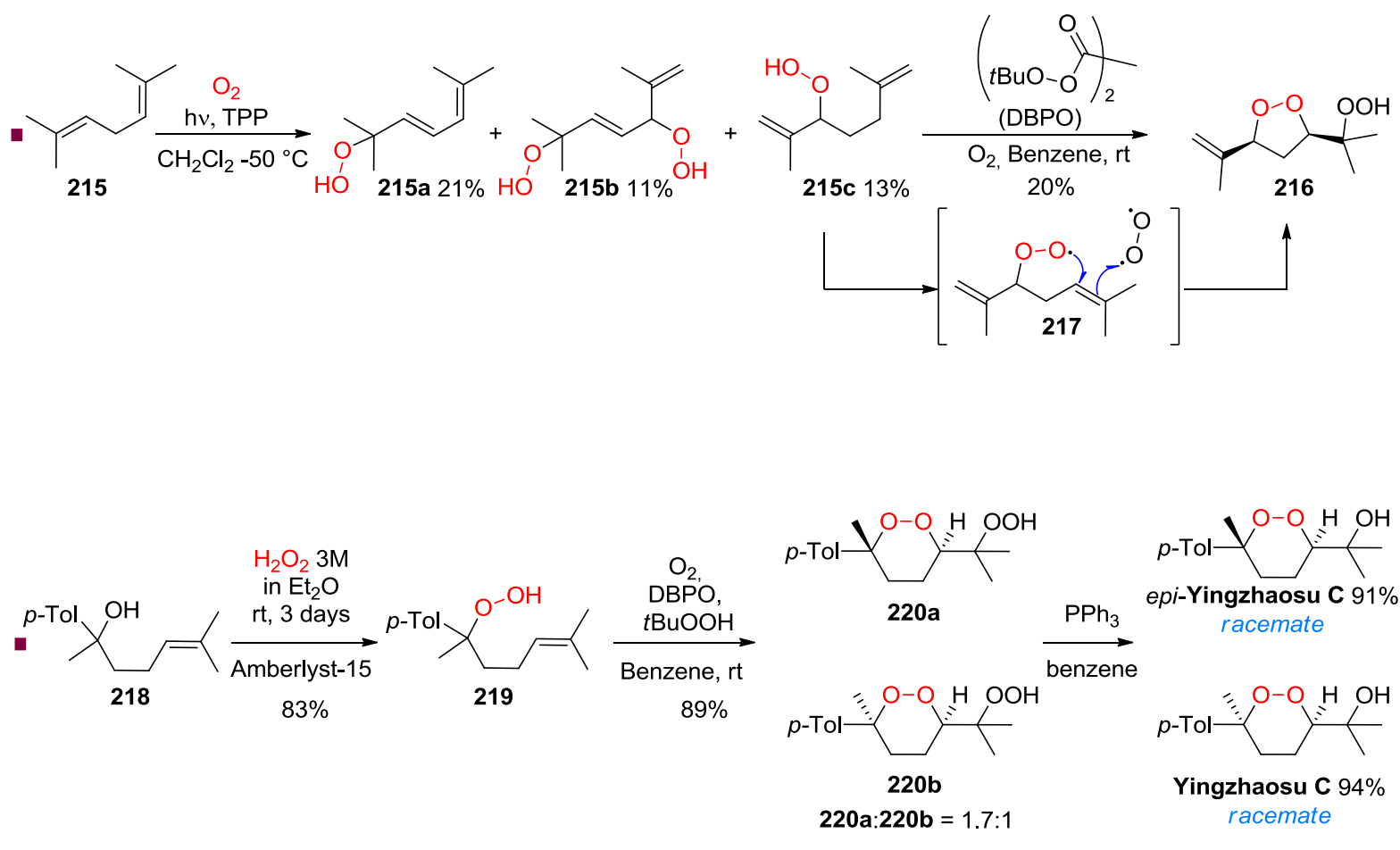

Scheme 32

\subsection{Cyclization through nucleophilic addition or substitution}

In contrast to free radical chain reactions, which can mostly provide 1,2dioxolane or 1,2-dioxane units directly from oxygen in one step, cyclizations using a nucleophilic addition or substitution are predominantly prepared from hydroperoxides. However, these methods are generally stereospecific and allow more easily the preparation of endoperoxides with controlled and defined stereochemistry. Few examples, presenting a direct formation of an endoperoxide from hydrogen peroxide or one of its derivatives, are in general a two-step sequence in a one-pot process. Synthesis of hydroperoxides was discussed in paragraph 3.2.2.1, but reactions presented in this section will be disclosed following their cyclization step. 


\subsubsection{Nucleophilic substitution}

\subsubsection{Nucleophilic substitution $\left(\mathrm{SN}_{2}\right)$ on halides or sulfonates}

Nucleophilic substitution by some hydrogen peroxide derivatives in alkylationtype reactions is complicated due to the low reactivity of peroxyanion in this transformation and the propensity of organic peroxides to degrade under basic conditions. Nevertheless, double alkylation from sulfonates was early studied by Corey and Nicolaou in 1975. They reported a first example of a direct formation of 1,2-dioxolane by using potassium superoxide and 18-crown- 6 ether to improve its solubility in DMSO.164 This reaction relies first on the formation of a peroxyradical derivative, which is reduced in situ in a peroxyanion that reacts further in an intramolecular fashion to displace the second leaving group. Thus, treatment of dimesylate 221 under these conditions at $25{ }^{\circ} \mathrm{C}$ for 3 minutes afforded cis-1,2dioxolane $\mathbf{2 2 2}$ in moderate yield (Scheme 33).

Following a similar strategy, several endoperoxides have been prepared from ditriflates and bis-(tributyltin)peroxide by Salomon and Salomon. ${ }^{165}$ Therefore, dioxolanes 224a-b and 1,2-dioxane 224c could be synthesized from compound 223a-b and 223c respectively, in acceptable yields. These two examples showed the utilization of more reactive sulfonates towards alkylation with oxygen derivatives and were applied to few substituted substrates (Scheme 33).

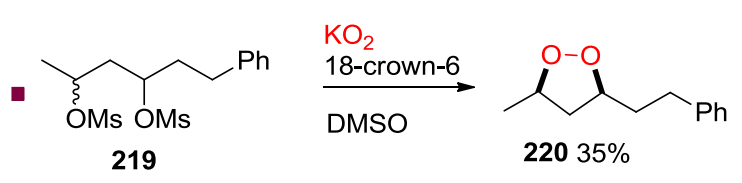

219
$22035 \%$

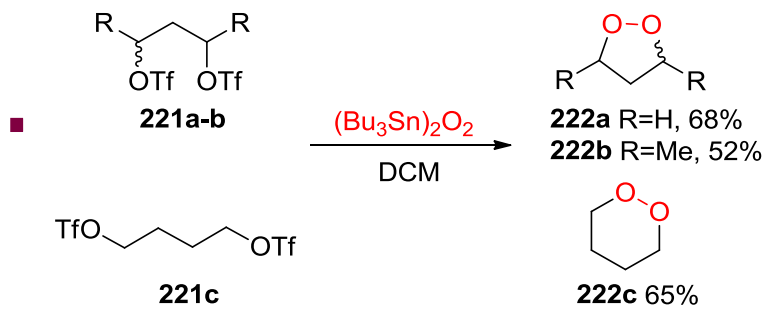

221c

\section{Scheme 33}

Use of hydroperoxides for further cyclization by alkylation was more widely applied because it circumvents the problem of the poorly effective intermolecular alkylation reaction, thus the hydroperoxide function could be introduced by a more convenient method. Furthermore, alkyl halides need generally to be activated by a silver salt to be effective alkylating agents toward the hydroperoxy function. Therefore, in a pioneer example, Bloodworth and coworkers managed to cyclize product 225 into 1,2-dioxolane 226a with endo configuration and inversion of configuration by using $\mathrm{Ag}_{2} \mathrm{O}$ for $63 \mathrm{~h}$, and 1,2-dioxolane 226b with exo configuration 
and retention of configuration by using silver trifluoroacetate for $1.5 \mathrm{~h} .{ }^{166}$ No yield was given for these transformations, however retention of configuration observed for the reaction with silver trifluoroacetate was discussed and authors proposed that formation of bromonium ion $\mathbf{2 2 7}$ might be probably at the origin of this unexpected process (Scheme 34).

Similarly, Kishi and coworkers used the same transformation on a cyclopentyl system in their studies towards prostaglandin endoperoxide $\left(\mathrm{PGH}_{2}\right)$. An oxidative hydroboration was performed on cyclopentene $\mathbf{2 2 8}$ to give hydroperoxide $\mathbf{2 2 9}$. Intramolecular alkylation of the latter with $\mathrm{Ag}_{2} \mathrm{O}$ afforded endoperoxide $\mathbf{2 3 0}$ in $58 \%$ yield (Scheme 34).167,168
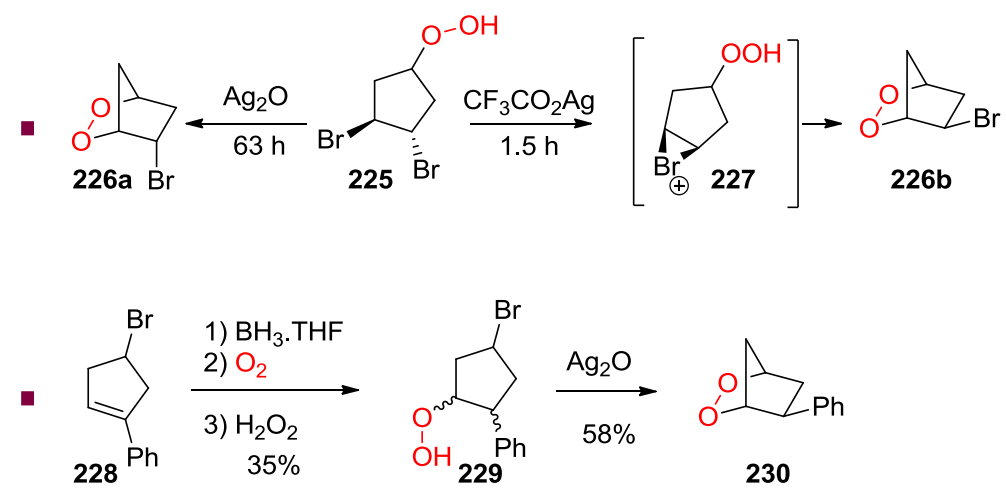

\section{Scheme 34}

More recent work by Dussault and coworkers showed that a double cyclization could be performed from hydroperoxyketals. ${ }^{169}$ Notably, dimesilate 231 was transformed in ketone 232, but not in hydroperoxyketal as expected. Further reaction with $\mathrm{H}_{2} \mathrm{O}_{2}$ and $\mathrm{H}_{2} \mathrm{SO}_{4}$ afforded directly spiro-compound 233 . Homologated substrate 233, in contrast, could be converted directly by ozonolysis in hydroperoxyketal 235. However no spontaneous cyclization was observed in this case. Treatment with $t$-BuOK and 18-crown-6 ether, however, could lead to expected structure $\mathbf{2 3 6}$ (Scheme 35).

Application of these last conditions to the total synthesis of peroxyplakoric acids, and in particular A3 derivative, allowed the construction of the 1,2-dioxane unit. ${ }^{170}$ Indeed, intermediate $\mathbf{2 3 7}$ underwent an ozonolysis in presence of methanol to furnish hemiperoxyketal 238. Cyclization with $t$-BuOK and 18-crown-6 ether afforded in good yield 1,2-dioxane 239. Further fonctionalizations including a hydrozirconation, a Negishi cross-coupling and a deprotection afforded ultimately 
compound 240. Unfortunately authors could not complete their final objective (Scheme 35).
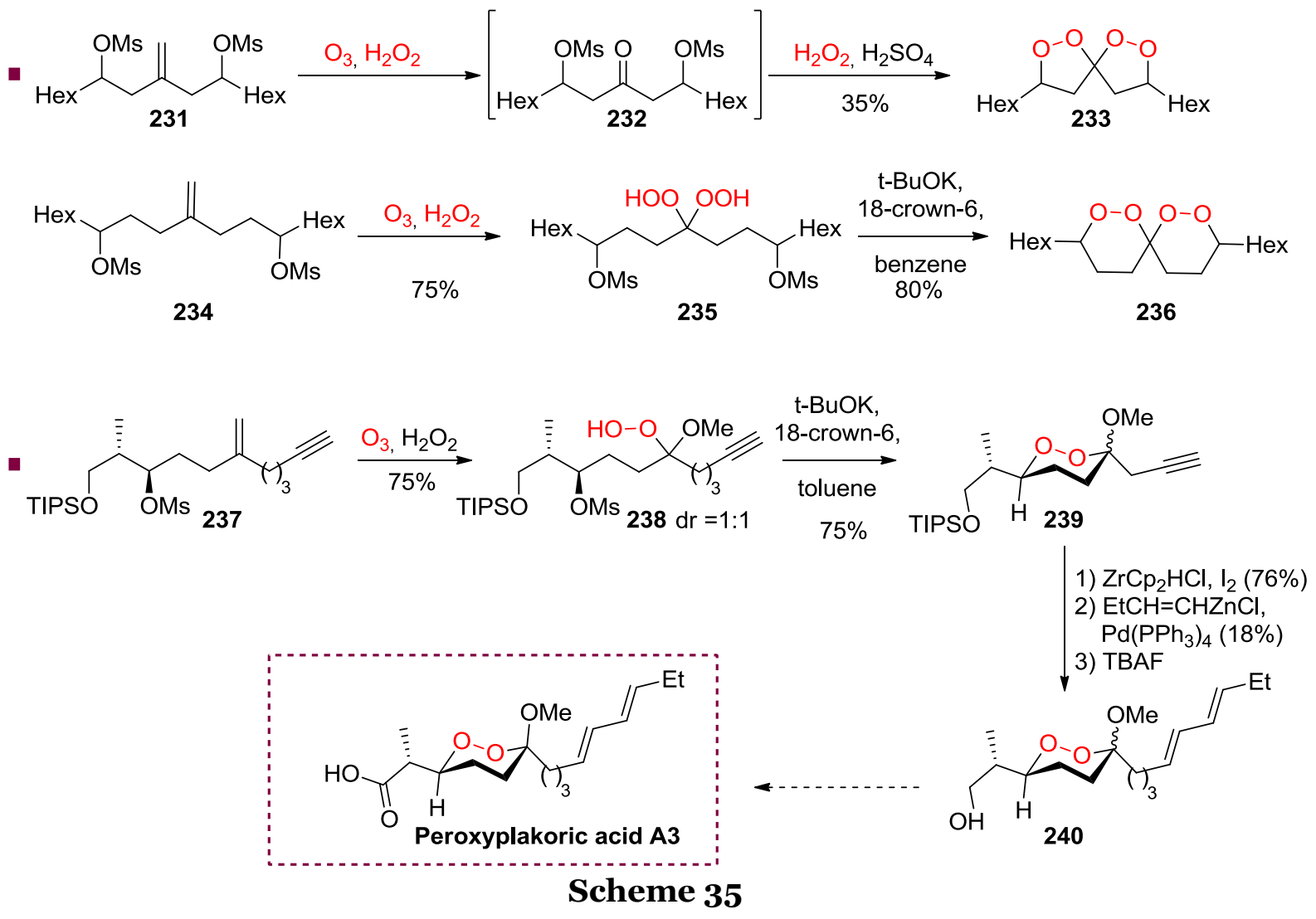

Lastly was reported the total synthesis of dicarnoxide C by Seifert, and their key step for the construction of the 1,2-dioxolane unit was also an intramolecular alkylation. ${ }^{171}$ Authors performed first an Isayama-Mukaiyama cobalt catalyzed hydroperoxy-silylation on compound $\mathbf{2 4 1}$ followed by a fluoride treatment to remove the silyl group and install the hydroperoxide function. Then the cyclization of 242 was achieved with DBU as base at $60^{\circ} \mathrm{C}$. Functionalization of 243 by introducing the fatty sesquiterpene part, using a Julia-Kochiensky olefination and further functional modifications, afforded Diacarnoxide $\mathrm{C}$ as a mixture of diastereomers. Indeed, introduction of the hydroperoxyl function could not be controlled and authors were unable to separate the different diastereomers at any stage of the synthesis. However most of the analytical data matched with those of the natural product (Scheme 36). 


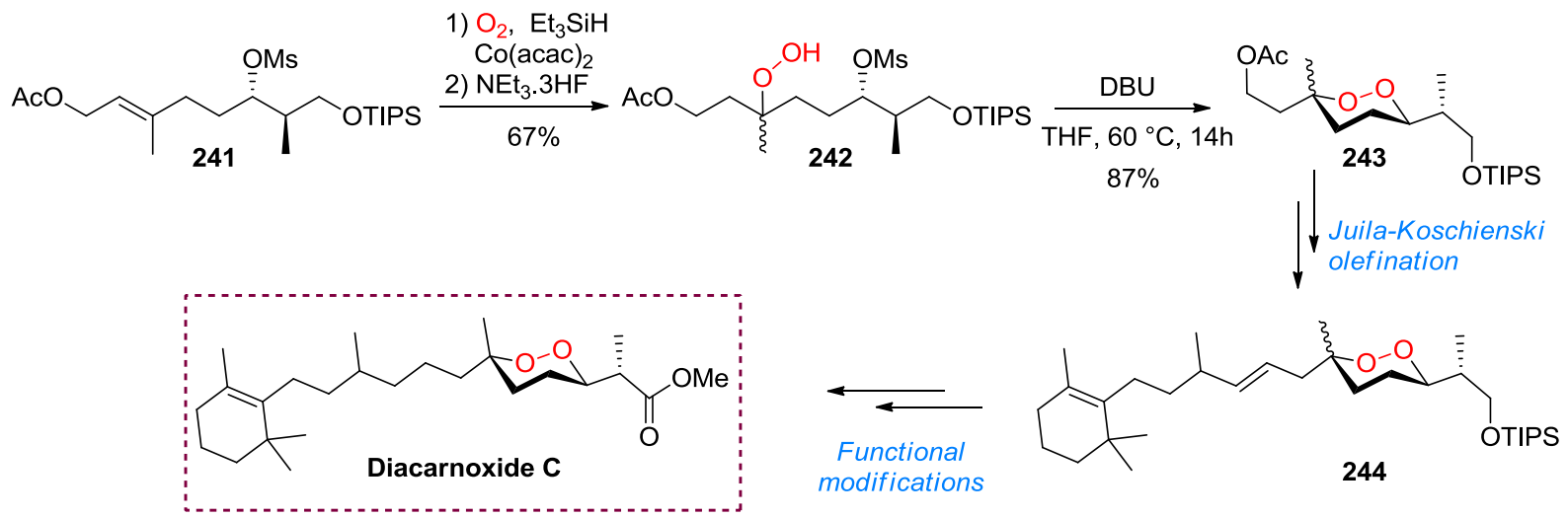

Scheme 36

3.3.1.2 Nucleophilic substitution $\left(\mathrm{SN}_{1}\right)$ via a carbocation.

Preparation of 1,2-dioxolane can be also achieved through a $\mathrm{SN}_{1}$ pathway. So, in this example, allylic alcohol 245 was transformed into allylic hydroperoxides $\mathbf{2 4 6 a}$ and 246b in a 10:90 ratio by using an ene-reaction with singlet oxygen. The mixture was then treated with a mild Lewis acid such as $\operatorname{In}(\mathrm{OTf})_{3}$ to drive to $\mathbf{2 4 7}$ through carbocation $\mathbf{2 4 8}$ (Scheme 37).172

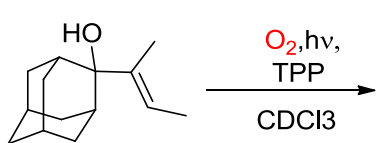

245



$246 a$

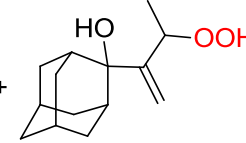

246b

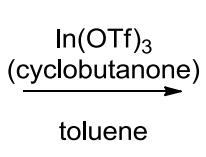
$15 \%$ (2 steps)<smiles>[Z16]C1C2CC3CC1C(C2)C3C(=C)C(C)O</smiles>



247

\section{Scheme 37}

This mechanism takes also part in the total synthesis of propindilactone G. ${ }^{173}$ Thus, hydroperoxysilane $\mathbf{2 5 0}$ could be obtained via an Isayama-Mukaiyama hydroperoxysilylation of $\mathbf{2 4 9}$ and cyclization on hydroxyl group was then performed using $\mathrm{BF}_{3} \cdot \mathrm{OEt}_{2}$. A complete inversion of the stereochemistry in $\mathbf{2 5 1}$ was observed due to the orientation of the peroxy group in 250. Eleven steps were then necessary to obtain propindilactone G. It is also noteworthy that intermediate $\mathbf{2 5 1}$ is closely related to pseudolarolides as in Figure 5 (vide supra). (Scheme 38) 


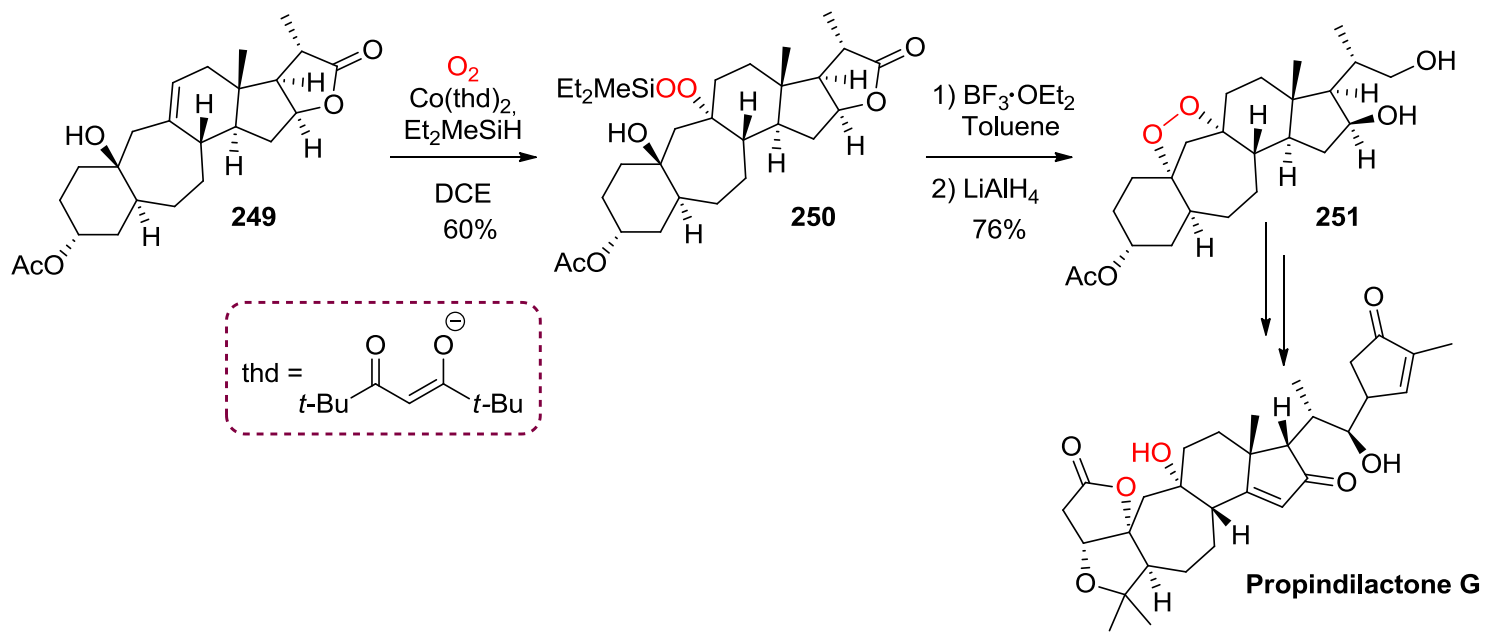

Scheme 38

\subsubsection{Nucleophilic substitution on strained heterocycles}

Epoxides and oxetanes are convenient intermediates, which can easily undergo a nucleophilic substitution with hydrogen peroxides or any hydroperoxide after activation of the heterocycle with a Lewis or Bronsted acid. The advantages of this method are the possibility to also introduce a hydroxyl group in $\alpha$ or $\beta$ position of the peroxide function with acids conditions, compared to the basic conditions, and the availability of numerous methods to obtain enantiopure epoxides and in less extend oxetanes, which makes it possible to obtain endoperoxides with a good control of the stereochemistry.

$\mathrm{Xu}$ and coworkers applied early this method to assign both relative and absolute configuration of Yingzaosu C. Indeed, since chiral epoxides are readily available in the two enantiomeric forms by using titanium catalyzed asymmetric Sharpless epoxidation, authors used this method for preparing the four possible stereoisomers of Yingzhaosu C.61,174 Thus, compound 252 was transformed into mixture of diastereomers 253a-b and 253c-d after a Sharpless epoxidation with one or the other enantiomer of diisopropyl tartrate (DIPT), acetylation of the primary alcohol, Isayama-mukaiyama hydroperoxydation and desilylation. It is noteworthy that the hydroperoxidation brings a mixture of diastereomers and that authors first tried to cyclize directly the hydroperoxides through formation of a peroxy anion upon desilylation, but no epoxide ring opening was observed under these conditions, with KF. However acid-mediated conditions allowed cleanly cyclization, affording 1,2dioxanes 254a-d. At this step, the different diastereomers could be separated by chromatography and after three more steps performed individually on each products, 
the four different possible stereoisomers of yingzhaosu $\mathrm{C}$ were thus obtained. Optical rotation study allowed authors to conclude that natural yingzhaosu $\mathrm{C}$ is a mixture of enantiomers with the (+)-one being predominant. However they could not conclude themselves about the relative stereochemistry of yingzhaosu $\mathrm{C}$ because they could not determine which synthetic product was the cis or trans isomer, but slightly later Boukouvalas and coworkers ${ }^{163}$ (see Scheme 31) could in contrast conclude that the cis isomer is the natural product (stereoisomers a and d). (Scheme 39)

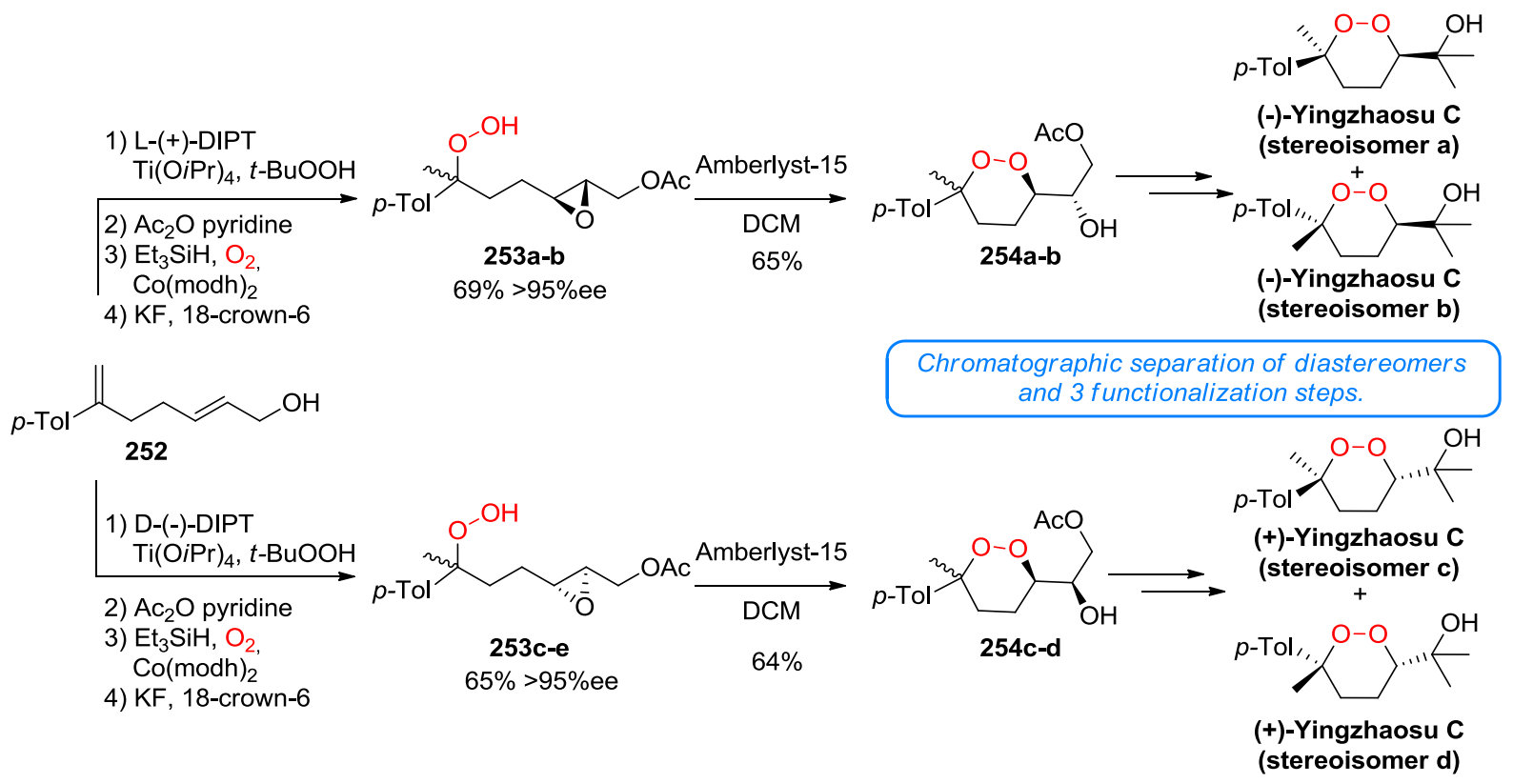

\section{Scheme 39}

Gemma, Campiani and coworkers developed also strategy based on a hydroperoxidation of olefin $\mathbf{2 5 5}$ followed by an epoxide ring-opening for the total synthesis of dihydroplakortin and 6-epi-dihydroplakortin. ${ }^{175}$ After a synthetic study, ${ }^{176}$ they were able to access natural products by cyclizing the mixture of diastereoisomer 256 into compounds $(6 S)$-257 and (6R)-257, which were both separated by chromatography at this step. A 5 steps sequence, i.e. an oxidative cleavage of diol, homologation through Wittig reaction, oxidation and esterification, furnished both of desired compounds: dihydroplakortin and its 6-epimer. (Scheme 40) 


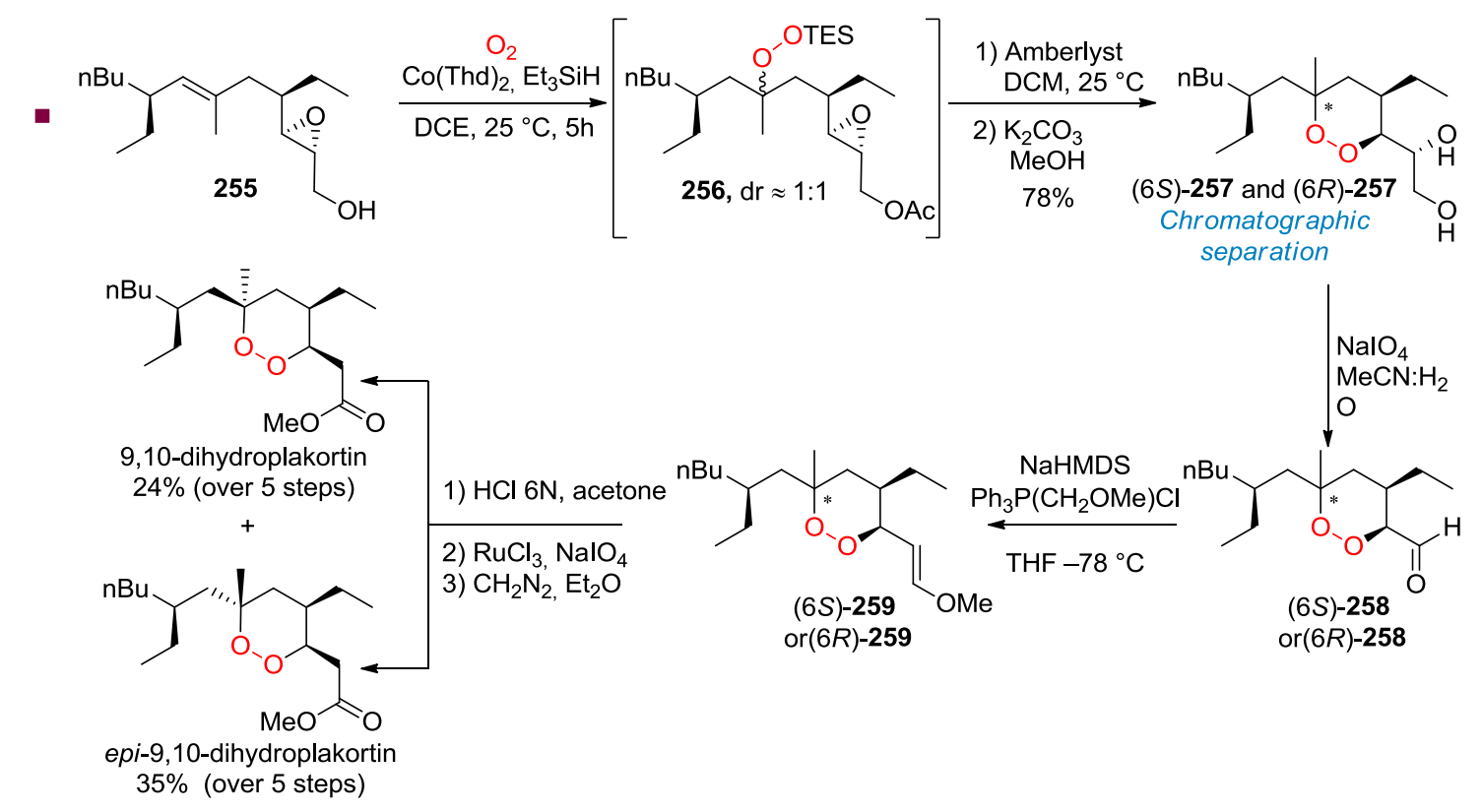

\section{Scheme 40}

The strategy based on epoxide ring-opening was also applied by Vatèle and coworkers in two different works towards the total synthesis of endoperoxides. Firstly, epoxy-alcohol 253 underwent a hydroperoxidation to give compound 254 (as a mixture of diastereomers) and an acid catalysis allowed the formation of 1,2dioxane 255, a hypothetical precursor in their work towards total synthesis of Plakortolide E. ${ }^{177}$ In a second study towards total synthesis of andavadoic acid, the same strategy was exactly applied through hydroperoxydation of $\mathbf{2 5 6 .}{ }^{26}$ However the conditions used and the regioselectivity of the oxirane ring opening were different to obtain 258a-b. Indeed, a 5-exo-tet cyclization was observed in contrast to a 6-endotet in the case of the transformation of $\mathbf{2 5 4}$ into $\mathbf{2 5 5}$, and some basic conditions were also preferred (Scheme 41). 

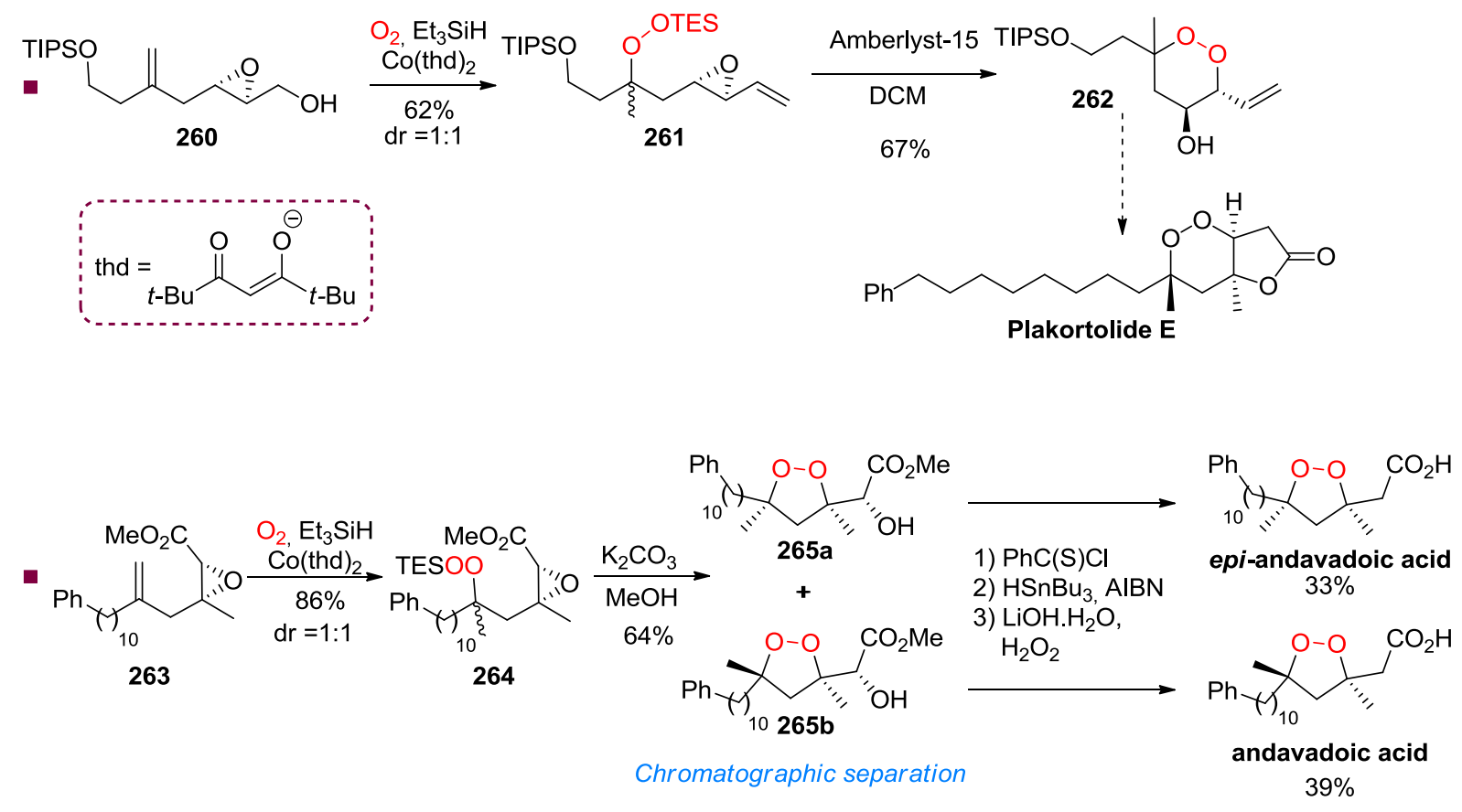

\section{Scheme 41}

Epoxides were also used in a cyclization cascade towards the total synthesis of stolonoxides A-F. Deng and coworkers were first interested by applying their enantioselective catalytic peroxidation of enal from 266 with aminoquinidine organocatalyst 268 and hydroperoxide 269. ${ }^{178}$ After transformation of the aldehyde into a methyl ester, peroxyketal $\mathbf{2 6 7}$ was obtained with 90\% of the desired diastereomer. Protecting group switch of the peroxyether function followed by Shi epoxidation afforded intermediate 270. Cleavage of the protecting group followed by a base catalyzed ring opening cascade of the two epoxides, afforded endoperoxide $\mathbf{2 7 2}$ in $52 \%$ yield. The direct use of the peroxyketal present in $\mathbf{2 6 7}$ did not allow authors to continue the synthesis because of some side-reactions during the deprotection step and an acid-catalyzed ring opening of the epoxides drove to a poor yield for 272 (15\%) along with some bis-THF byproducts, which correspond to a cascade cyclization from a reduced version of hydroperoxide 270. (Scheme 42) 


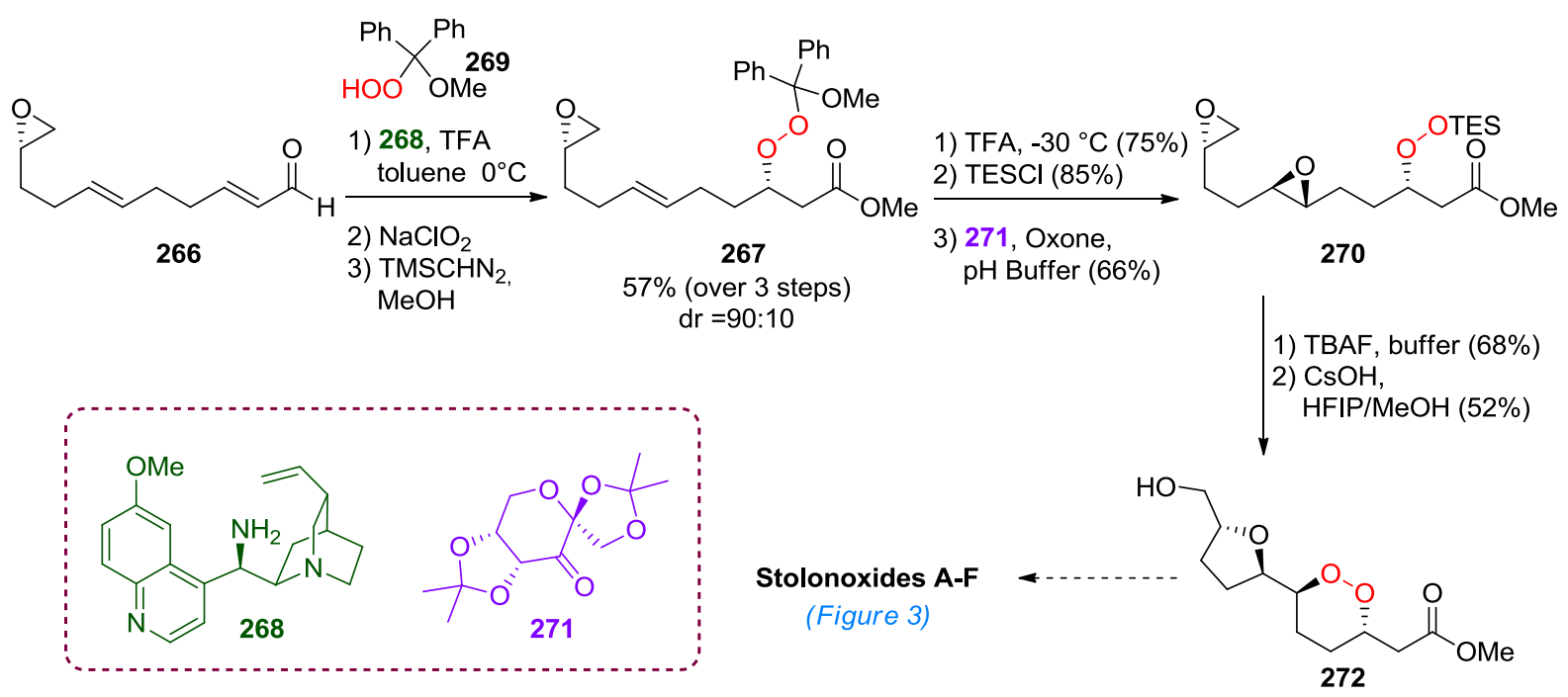

Scheme 42

Cyclization of hydroperoxides could be also conducted with oxetanes. Dussault and coworkers showed this process is applicable to any oxetane such as compounds 273a-d to give rise to 1,2-dioxolones $274 a-d .{ }^{179}$ The reaction takes place on the more substituted position with generally an inversion of the configuration. When the reaction was conducted with homologated compounds 273e-f, 1,2-dioxanes 274e-f were obtained. However, conditions had to be optimized and reaction time was also extended from 1-2 h to 2 days, meaning 6-exo-tet cyclization is much slower. An erosion of the stereoselectivity was also observed for compound 274f, since partial formation of a carbocation could have arisen in this case (Scheme 43).

Cyclization was also accomplished from hemiperoxyketals. Thus, olefins 275a-c underwent an ozonolysis to generate this function. Surprisingly, authors isolated directly 1,2-dioxolanes 276a-c without the help of any acid catalysis. However, this transformation was difficult to apply to the synthesis of the corresponding 1,2-dioxanes. ${ }^{179}$ (Scheme 43). 


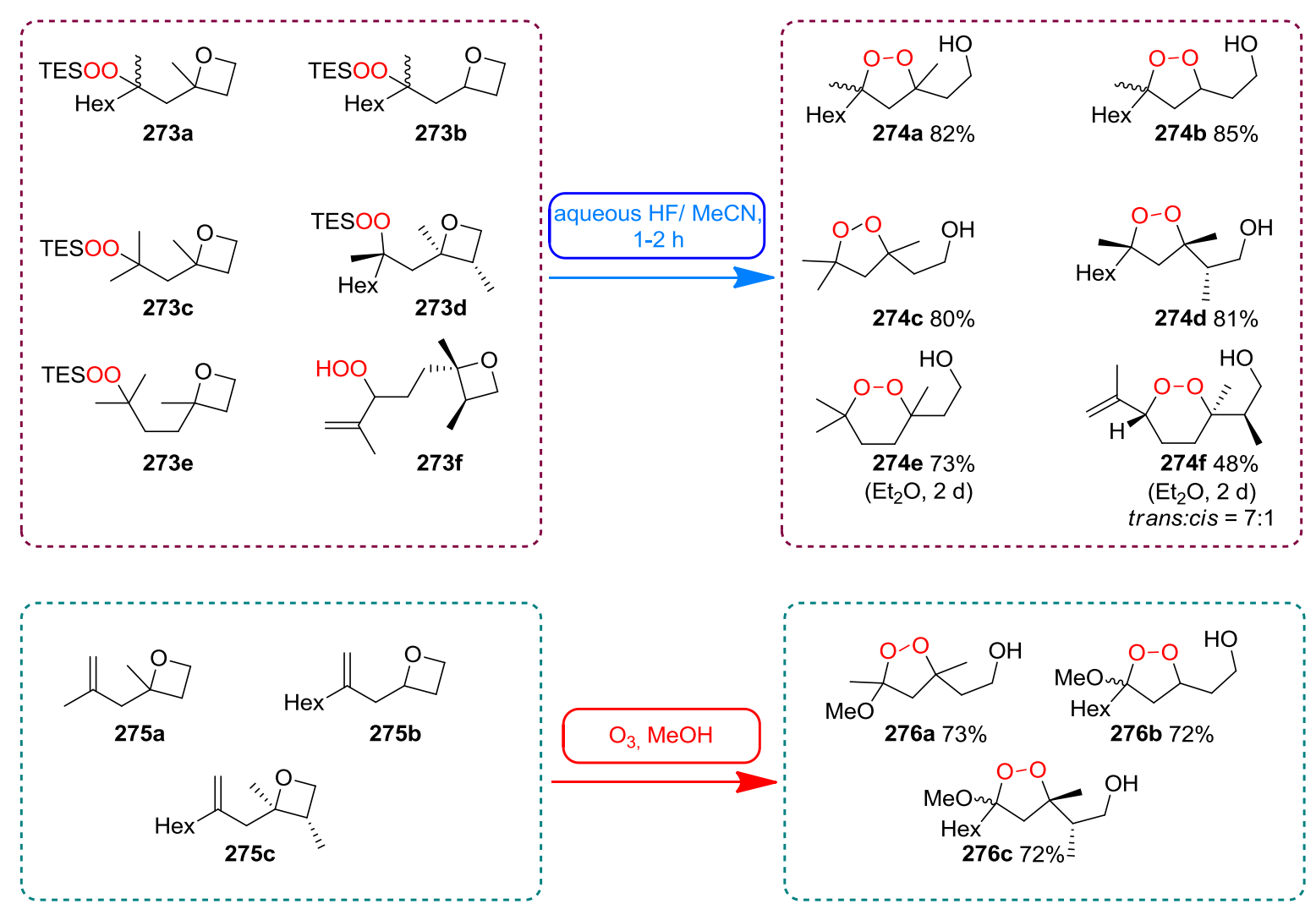

Scheme 43

\subsubsection{Addition to carbon-carbon double bonds and related functions}

\subsubsection{Peroxymercuration}

Mercury is metal well-known for efficiently promoting nucleophilic addition on olefins or alkynes, because of its high carbophilicity, which means this metal can give an electrophilic character to any $\pi$-bond system. The process was also applicable to hydrogen peroxide or hydroperoxide through a peroxymercuration. Pioneer work from Porter and coworkers showed the interest of this transformation for the synthesis of 1,2-dioxanes or 1,2-dioxolanes from hydroperoxides containing an olefin in $\gamma$ or $\delta$-position. ${ }^{180}$ Mercurations are mechanistically related to halogenations, and the metal stays incorporated in final product unless any cleavage of the carbonmercury bond is undertook. Moreover, mercury and its salts are known to be highly toxic and due to stoichiometric amount involved in the process, no recent application was reported for any peroxycyclization.

However, the most noteworthy applications were probably achieved by Bloodworth and coworkers in the 80's where a double peroxymercuration from hydrogen peroxide and diene $\mathbf{2 7 7} \mathbf{a}-\mathbf{b}$ could be carried out, giving 1,2-dioxolane $\mathbf{2 7 8 a}$ or 1,2-dioxane $\mathbf{2 7 8 b}$. $^{181,182,183}$ Carbon-mercury bonds could be then cleaved either by 
reduction with $\mathrm{NaBH}_{4}$ and soda to give products 279a-b, while oxidation with bromine afforded products $\mathbf{2 8 0 a - b}$ (Scheme 44).

An application to the synthesis of some synthetic analogues of plakinic acids was reported from conjugated esters 281a-d. Thus, first peroxymercuration was performed through formation of $\mathbf{2 8 1 a - d}$ followed by a second one giving $\mathbf{2 7 3 a - d}$. A reductive demercuration delivered finally 1,2-dioxolanes $\mathbf{2 8 4 a - d}$ and after a final saponification, plakinic acid derivatives $\mathbf{2 8 5}$ a-d were obtained (Scheme 44). ${ }^{184}$
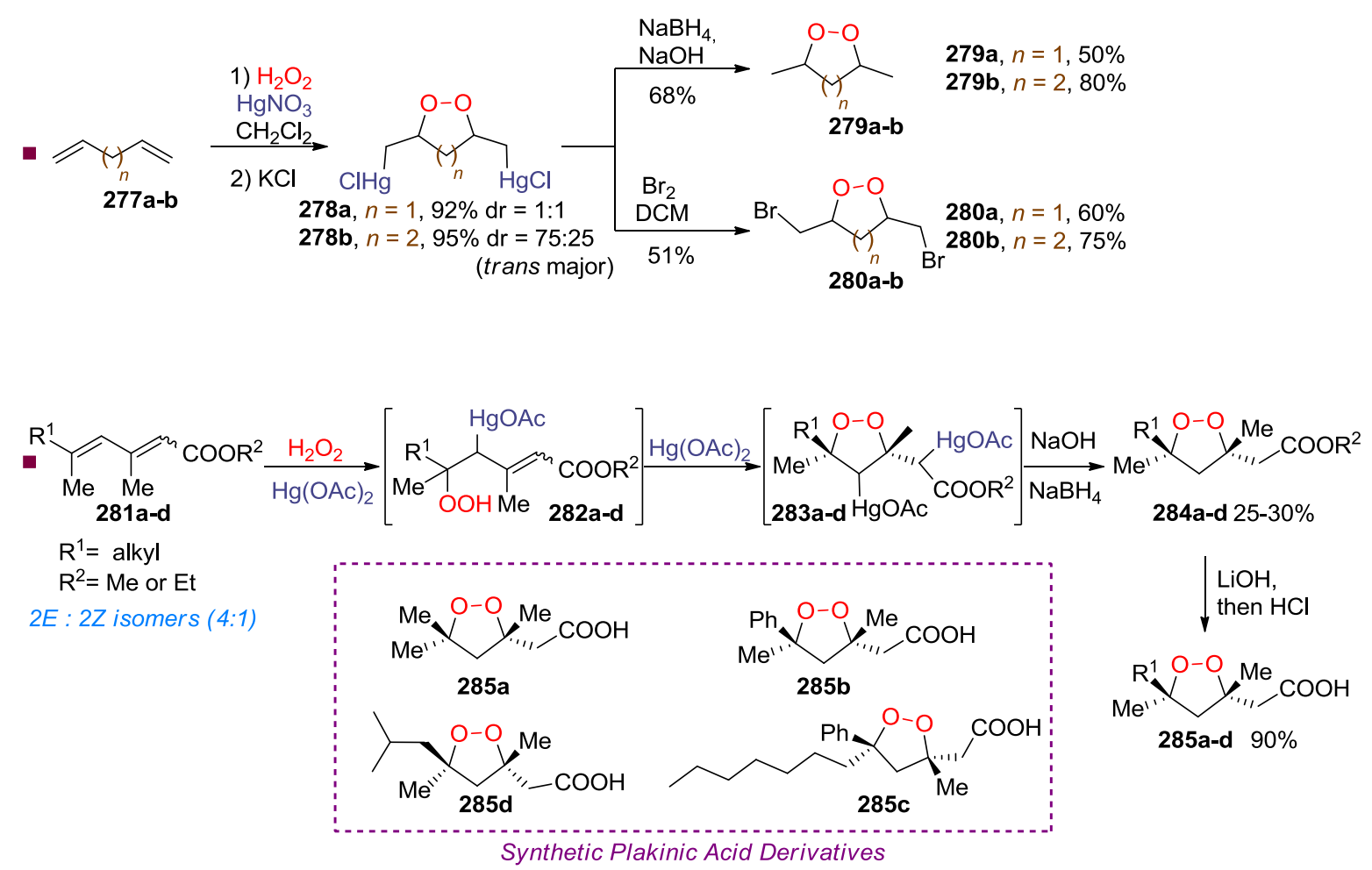

Scheme 44

Besides olefins, cyclopropanes possess also a $\pi$-bond character. Thus, a peroxycyclization from a hydroperoxide was reported on such substrates. Indeed hydroperoxides $\mathbf{2 8 7}$ underwent a cyclization in presence of perchloric acid and mercury(II) salts to give, in about $80 \%$ yield, organomercury compounds $\mathbf{2 8 8}$ as intermediates, isolated with a 60:40 cis:trans ratio. Reduction of the organomercury function with alkaline sodium borohydride afforded corresponding dioxolanes $\mathbf{2 8 9}$ in $52-60 \%$ yields (Scheme 45$).{ }^{185}$

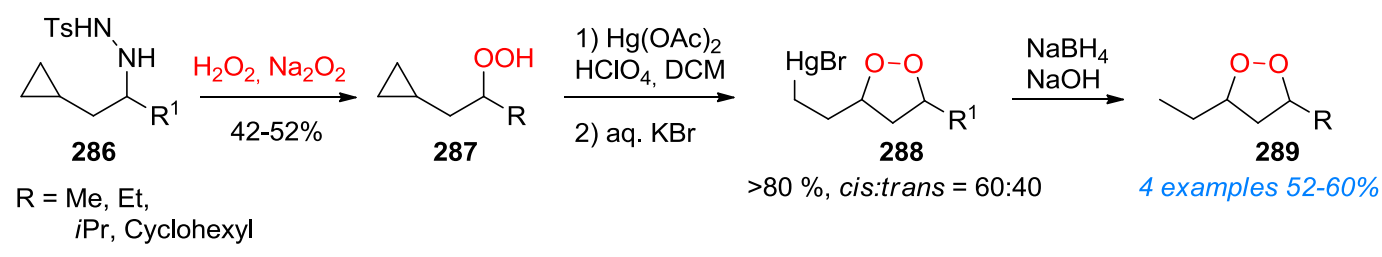

\section{Scheme 45}




\subsubsection{Peroxyhalogenation}

Like mercury cations, halides have the ability to activate an olefin through the formation of a halonium ion. Bloodworth and coworkers particularly studied this transformation and they found out that two mechanisms are in competition. ${ }^{186}$ For instance, reaction of hydroperoxide 290 with $N$-iodosuccinimide (NIS) furnished 291a as a 1:1 mixture of diastereomers, suggesting a radical mechanism through the formation of peroxyradical 292a. In contrast reaction with with $\mathrm{N}$-bromosuccinimide (NBS) afforded 1,2-dioxolane 291b in a 80:20 mixture of diastereomer, meaning the mechanism is mainly ionic, via bromonium $\mathbf{2 9 3} \mathbf{b}$, due to the stereospecificity of the ionic pathway. However, 291b being in a 80:20 mixture of diastereomers might come from a minor radical process such as for NIS. $N$-Chlorosuccinimide (NCS) was unreactive for such transformation, and optimized conditions with $t$-butyl hypochlorite and pyridine overcame the difficulties initially encountered, affording 291c (Scheme 46). ${ }^{187}$

Peroxyhalogenation was later used in many syntheses, such as cyclization of 294 to 1,2-dioxolane 295 with $\mathrm{Br}_{2}$; the stereospecificty of the reaction beeing total for this example. ${ }^{188}$ A cycloperoxyiodination was also described from hydroperoxide 297, synthesized from meldrum's acid derivative 296 by a copper mediated peroxidation. Authors used 1,3-diiodo-5,5-dimethylhydantoin (DIH) as iodonium source, which gave a good regioselectivity and diastereoselectivity for the formation of 1,2-dioxolane $\mathbf{2 9 8}$ (Scheme 46). ${ }^{189}$

A more recent example was reported by Das and coworkers on the haloperoxycyclization of oxazoles. ${ }^{190}$ Thus, derivatives 299 were transformed into hydroperoxides 300 by a $\mathrm{SN}_{1}$ type reaction and further peroxycyclizations were achieved using PIDA and zinc halide. PIDA is oxidizing in situ the bromide or chloride into corresponding chlorine or bromine cation source, making further cycloperoxyhalogenation possible to yield, for example, 301a or 302b. Among other halogen oxidant these conditions proved to be the most high yielding. However zinc iodide was unable to furnish the desired 1,2-dioxanes. Therefore, up to fifteen spiro1,2-dioxanes with different substituants on the oxazole were obtained (Scheme 46). 

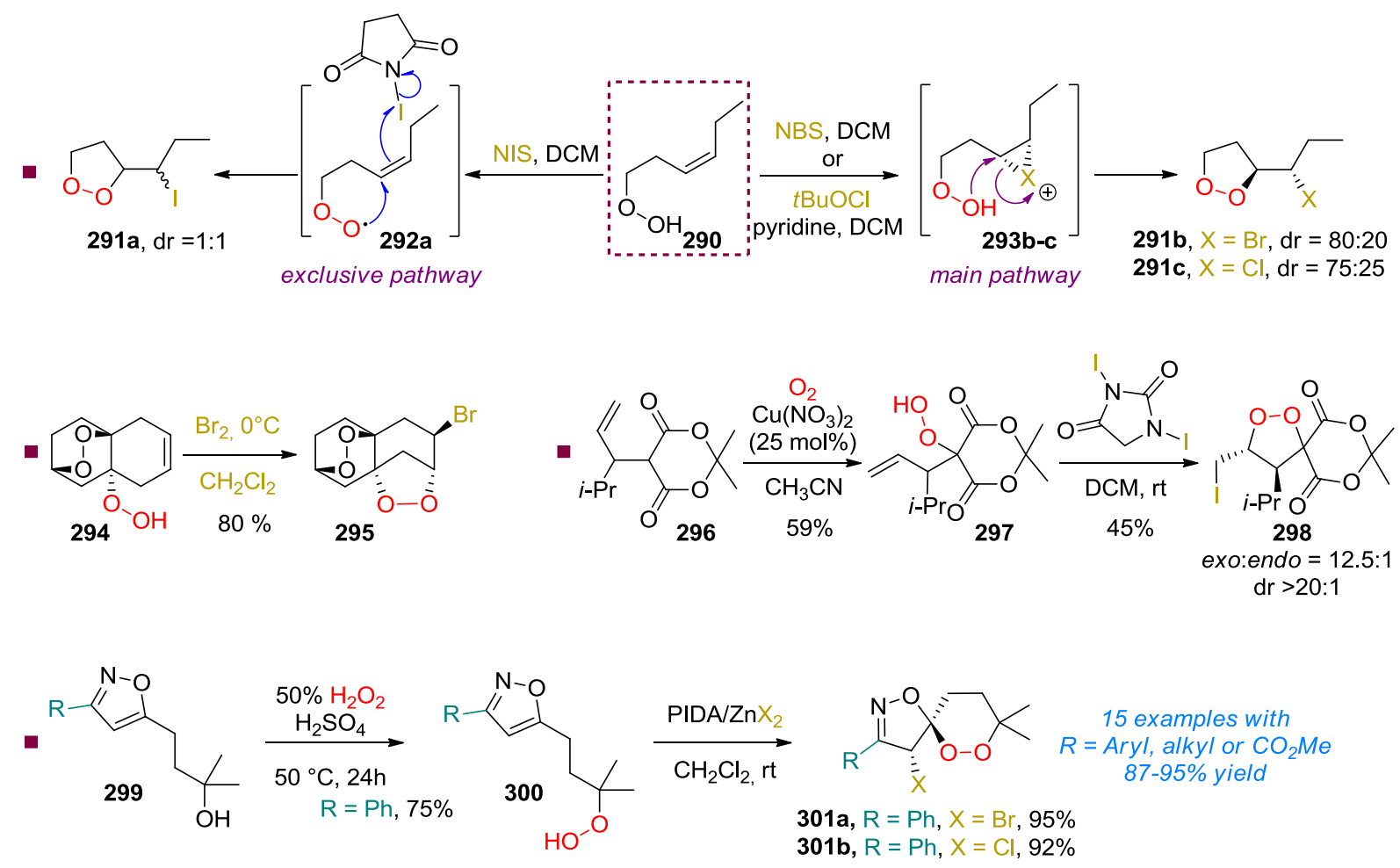

Scheme 46

\subsubsection{Metal catalyzed peroxycyclization}

Some recent methods are involving metal catalyzed peroxycyclization to produce 1,2-dioxanes or 1,2-dioxolanes. Palladium mediated reactions were particularly studied. Thus, Woerpel and coworkers presented a Waker-type reaction to transform hydroperoxyalkenes 3o2a-f into 1,2-dioxanes 303a-f. ${ }^{191}$ The reaction involves palladium(II) acetate with some benzoquinone to reoxidize the metal species. Indeed, such as in the Wacker oxidation, substrates 302a-f are coordinated to $\mathrm{Pd}(\mathrm{II})$ species in order to activate the olefin and perform an intramolecular addition from the hydroperoxide. Endoperoxides 303a-f were then obtained from intermediates 305, which undergo a $\beta$-elimination of the metal. Palladium(o) is then reoxidized in palladium(II) by the benzoquinone. The yield of this reaction is sometimes moderate, due to the hydroperoxide reduction by-products. A divergent protocol was also studied, using benzoquinone $10 \mathrm{~mol} \%$ and $\mathrm{AgCO}_{3}$ as an oxidant, however the yield could not really be improved, although it suppressed the reduction process, but gives instead other oxidation by-products (Scheme 47). 


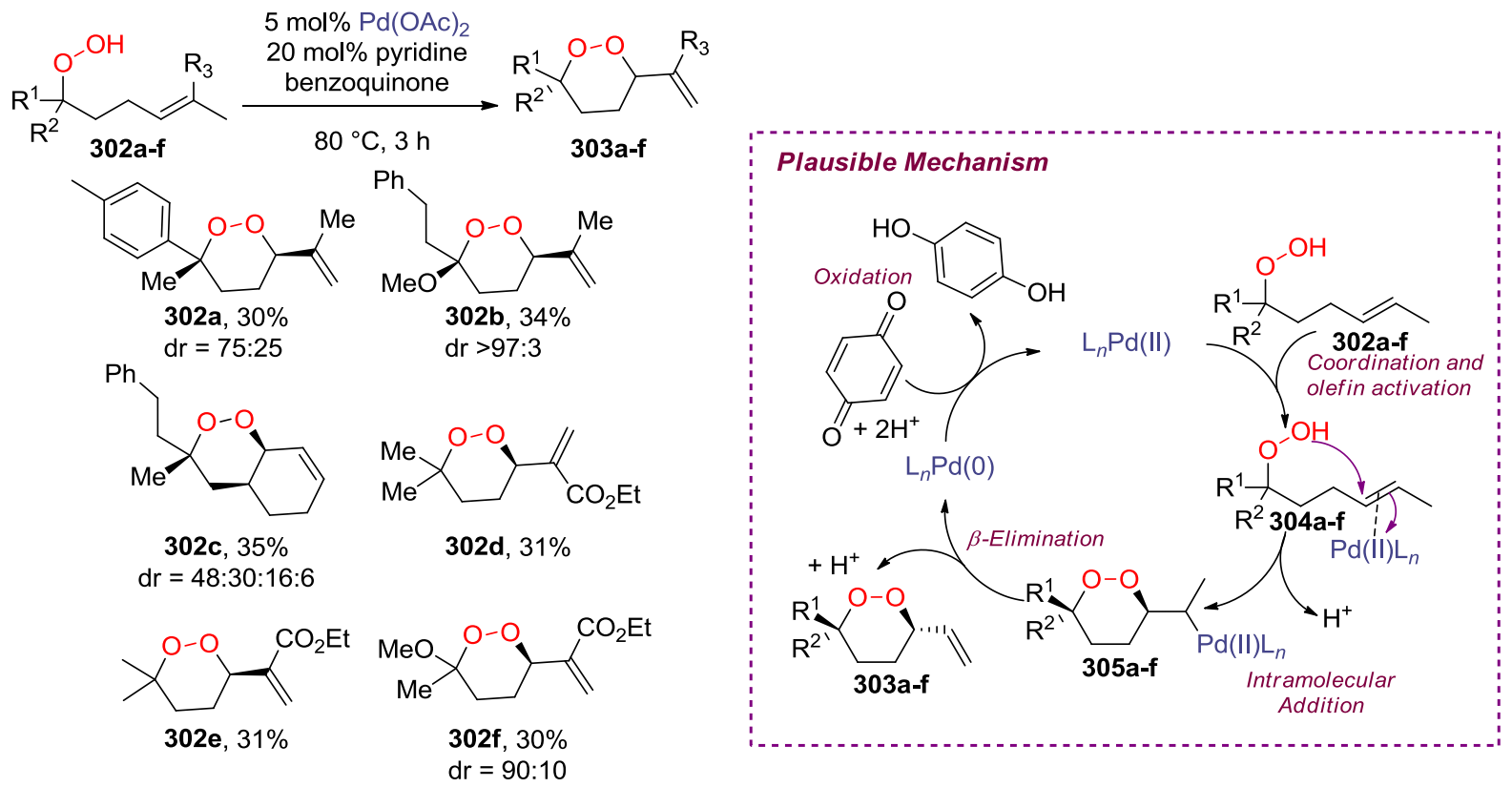

\section{Scheme 47}

Wong and coworkers, in their total synthesis of plakortide E, studied a synthetic methodology towards the palladium catalyzed oxidation of divinylcyclopropanes 306a-d into 1,2-dioxolanes 307a-d. ${ }^{148}$ After using molecular oxygen, which gave unsatisfactory results, they optimized the conditions using $\mathrm{Pd}\left(\mathrm{PPh}_{3}\right)_{4}$ and urea peroxide to obtain such endoperoxides. However the diastereoselectivty was poor and authors could not use this transformation in the total synthesis of plakortolide E (see also Chapter 3.2.1.6, Scheme 28). Although the transformation is original, it can be postulated that cyclopropanes are opened by $\operatorname{Pd}(\mathrm{o})$ species to give bis- $\pi$-allyl palladium species 309a-d, which can eventually be oxidized with oxygen peroxide into Pd(IV) species. A double Tsuji-Trost like process can then take place with hydrogen peroxide to furnish 1,2-dioxolane 307a-d. (Scheme 48) 


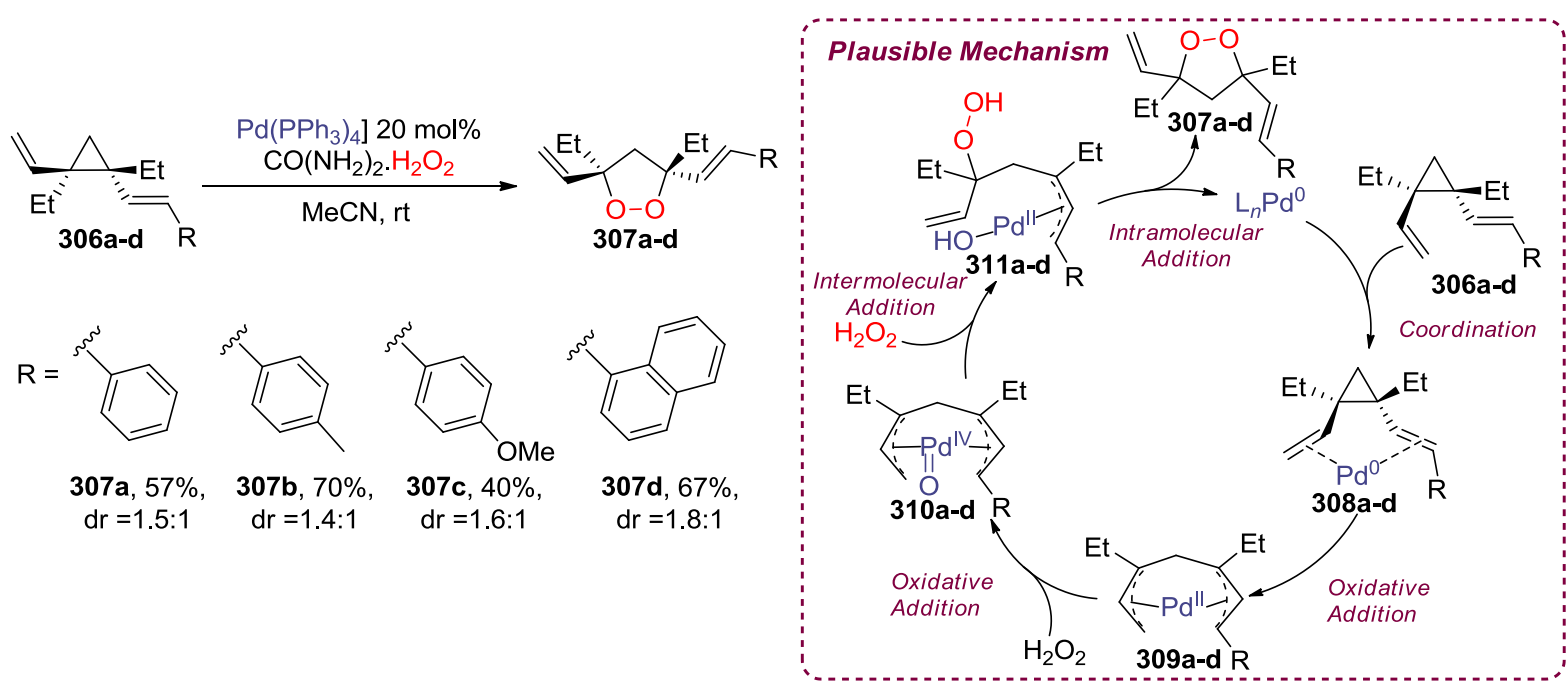

\section{Scheme 48}

Such as palladium, gold is known to behave an excellent carbophilicity. Thus, use of this metal was reported as a small application of a peroxycyclization. ${ }^{190}$ Meldrum's acid derivatives 312a-b were transformed into endoperoxyketals $\mathbf{3 1 3} \mathbf{a}-\mathbf{b}$ in presence of $\mathrm{PPh}_{3} \mathrm{AuNTf}_{2}$ and PPTS in MeOH. The first important step is the endodig peroxycyclization giving $\mathbf{3 1 5} \mathbf{a}-\mathbf{b}$. After a proto-demetallation, enol ether function in 316a-b was acetalized with methanol and PPTS. (Scheme 49)

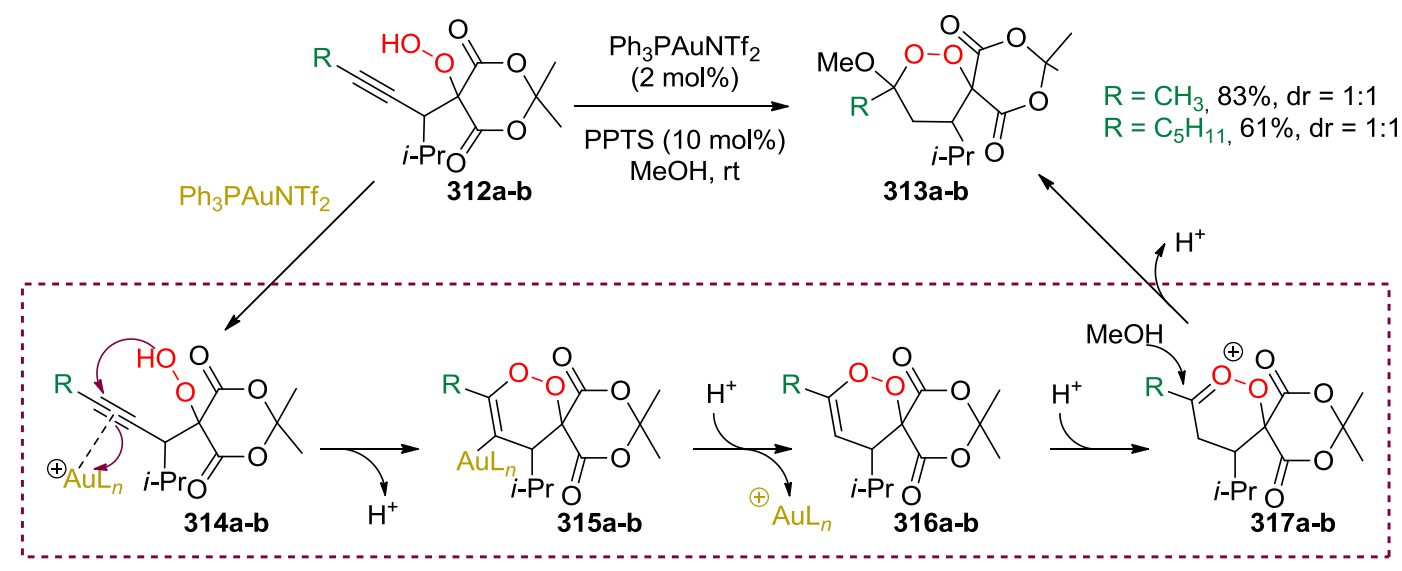

Scheme 49

\subsubsection{Conjugated addition}

Conjugate olefins with electron withdrawing groups are also potential electrophiles for conjugate addition of hydroperoxides, due to an electron density deficiency at the $\beta$-position. Addition of hydrogen peroxide to conjugate systems is a well-known old process, but generally results in the formation of epoxides, the reaction being known as Weitz-Scheffer epoxidation. ${ }^{192}$ This epoxidation is driven by the formation of an enolate just after the addition of the hydroperoxy anion, which 
can in turn react onto the electrophilic peroxide intramolecularly to give an oxirane. Thus, buffered or acidic conditions might preferentially keep the peroxide bond free.

Kobayashi M. and coworkers especially developed a methodology for the synthesis of 1,2-dioxanes such as $\mathbf{3 2 0}$ by using first $\mathrm{Sc}(\mathrm{OTf})_{3}$ mediated peroxyhemiacetalization of ketone $\mathbf{3 1 8}$ followed in second by peroxycyclization of 319 with diethylamine in trifluoroethanol.193,194,195 These conditions were particularly optimized for this transformation, the major by-products being the oxirane through Weitz-Scheffer epoxidation ${ }^{192}$ or a ketone through a Kornblum-DeLaMare rearrangement. ${ }^{196}$ Indeed, a base seems essential to induce a good nucleophilicity to the hydroperoxide for the addition onto the conjugated ester, but it also promotes these side reactions. Trifluoroethanol, because of its acidic character, slows them down, notably by buffering the reaction medium. (Scheme 50)

These reaction conditions were also applied in different syntheses of endoperoxides. Therefore, Dussault and coworkers used this peroxycyclization in the total synthesis of peroxycarnoates A\&D through the obtention of compound $\mathbf{3 2 1} ; 197$ $\mathrm{Wu}$ and coworkers obtained many different spiro-1,2-dioxanes such as $\mathbf{3 2 2}$ by using the same method;198,199,200 Ortega and coworkers also used this protocol for synthesis of 1,2-dioxolane $\mathbf{3 2 3}$ or 1,2-dioxane $\mathbf{3 2 4} ;^{201}$ Vatèle and coworkers used the Kobayashi's procedure in the final step of the synthesis of plakortolide $\mathrm{E}$ and (-)-entplakortolide $\mathrm{E} ;{ }^{202}$ and finally Harwood and coworkers also applied the $\mathrm{Et}_{2} \mathrm{NH}$ catalyzed peroxyclization method in the final step of the total synthesis of mycaperoxide B. ${ }^{203}$ It can be noted that all yields are from poor to modest in this transformation, due to the above mentioned side-reactions and many protocols needed to be reoptimized on certain substrates to afford desired endoperoxides (Scheme 50). 


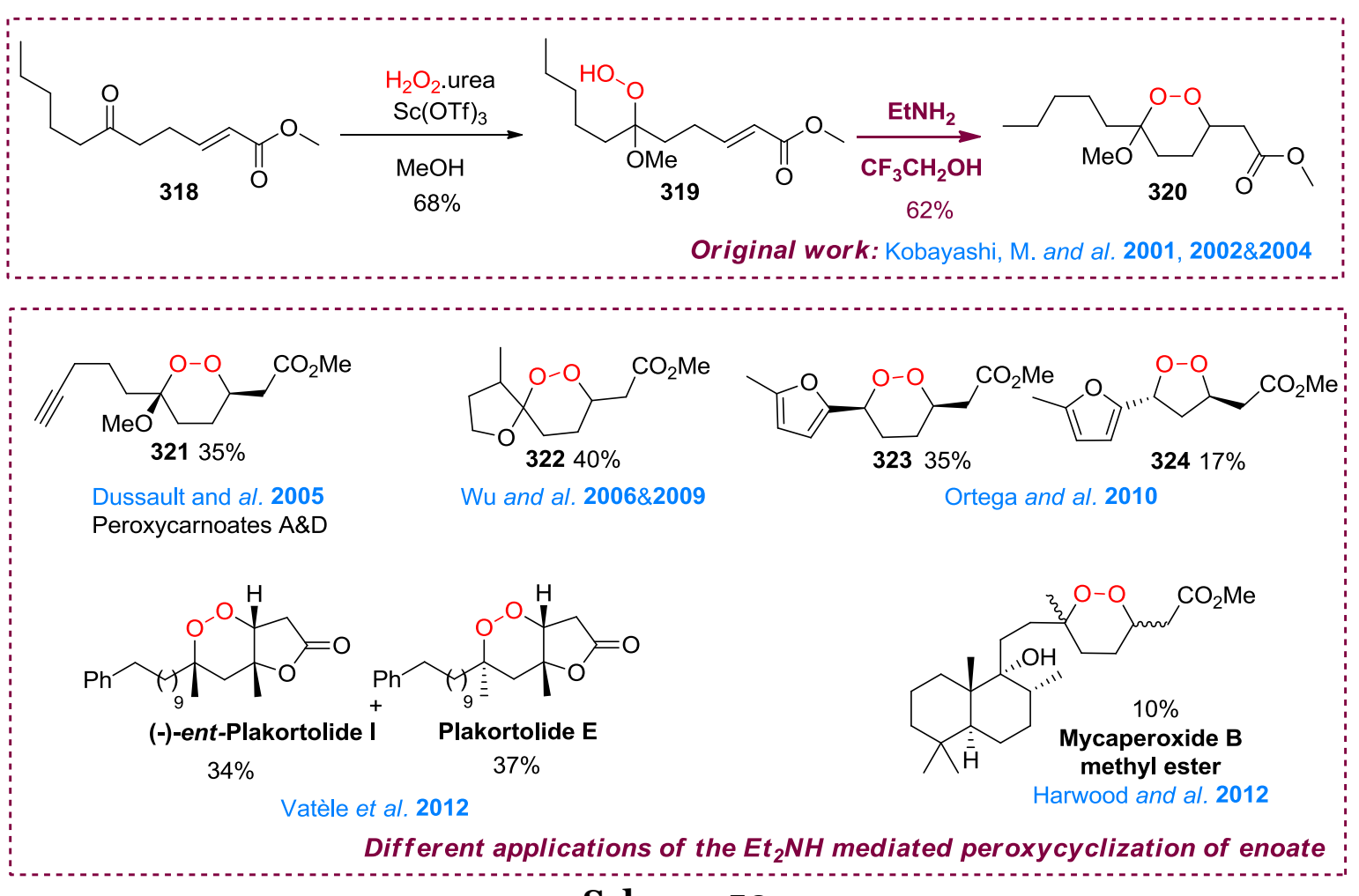

Scheme 50

Peroxycylization was also applied to other electron-withdrawing groups than esters. In these cases, $\mathrm{Et}_{2} \mathrm{NH}$ catalyzed peroxycyclization in trifluoroethanol was not always necessary. Indeed, after peroxyhemiacetalization of compounds $\mathbf{3 2 5} \mathbf{a}-\mathbf{b}, \mathbf{1 , 2}-$ dioxanes 326a-b were obtained spontaneously but in poor yields. ${ }^{195}$ Interestingly, conjugated nitro substrate $\mathbf{3 2 8}$ drove also spontaneously to 1,2-dioxane $\mathbf{3 3 0}$ in modest yield after a Sc(OTf) ${ }_{3}$ mediated peroxyhemiacetalization. ${ }^{199,200}$ These two last results suggest that peroxycylization was promoted by Lewis acid catalysis with these two different types of substrates (Scheme 51).

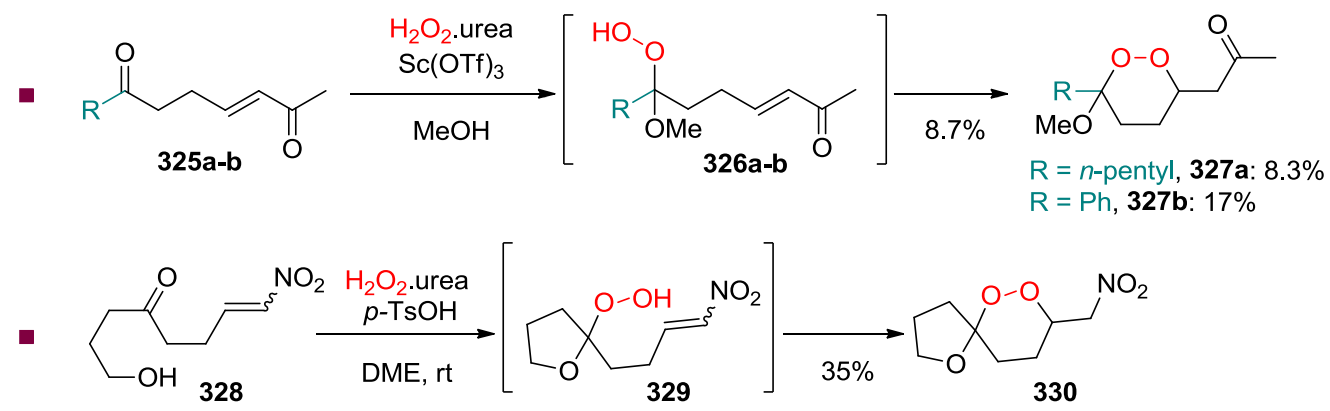

\section{Scheme 51}

A very significant work about conjugate addition was accomplished by List and coworkers. Indeed, they succeeded in preparing enantioselectively, among nineteen examples, various 1,2-dioxolan-3-ols of type 332 from enones of type 331, and more 
selectively with respect to the Weitz-Scheffer peroxidation driving to oxiranes of type 333. ${ }^{204,205}$ Organocatalysis is mediated by aminoquinine 268, in presence of trichloroacetic acid. Addition of hydrogen peroxide to the enone passes in fact through the formation of conjugated iminium ion 334, which is more reactive towards nucleophilic addition. Adduct $\mathbf{3 3 5}$ evolves back to ketone function, which drove then to dioxolane 332. Some representative examples such as 332a-d show that this reaction makes it possible to introduce different functionalities with excellent enantioselectivities, which is currently not so frequent in the synthesis of endoperoxides. However, the reaction seems limited to poorly functionalized ketones (for $\mathrm{R}^{2}$ ), because groups other than methyl give 1,2-dioxolanes with poor yields and a significant amount of oxiranes $\mathbf{3 3 3}$. We also personally experienced no conversion with more functionalized ketones (Scheme 52).

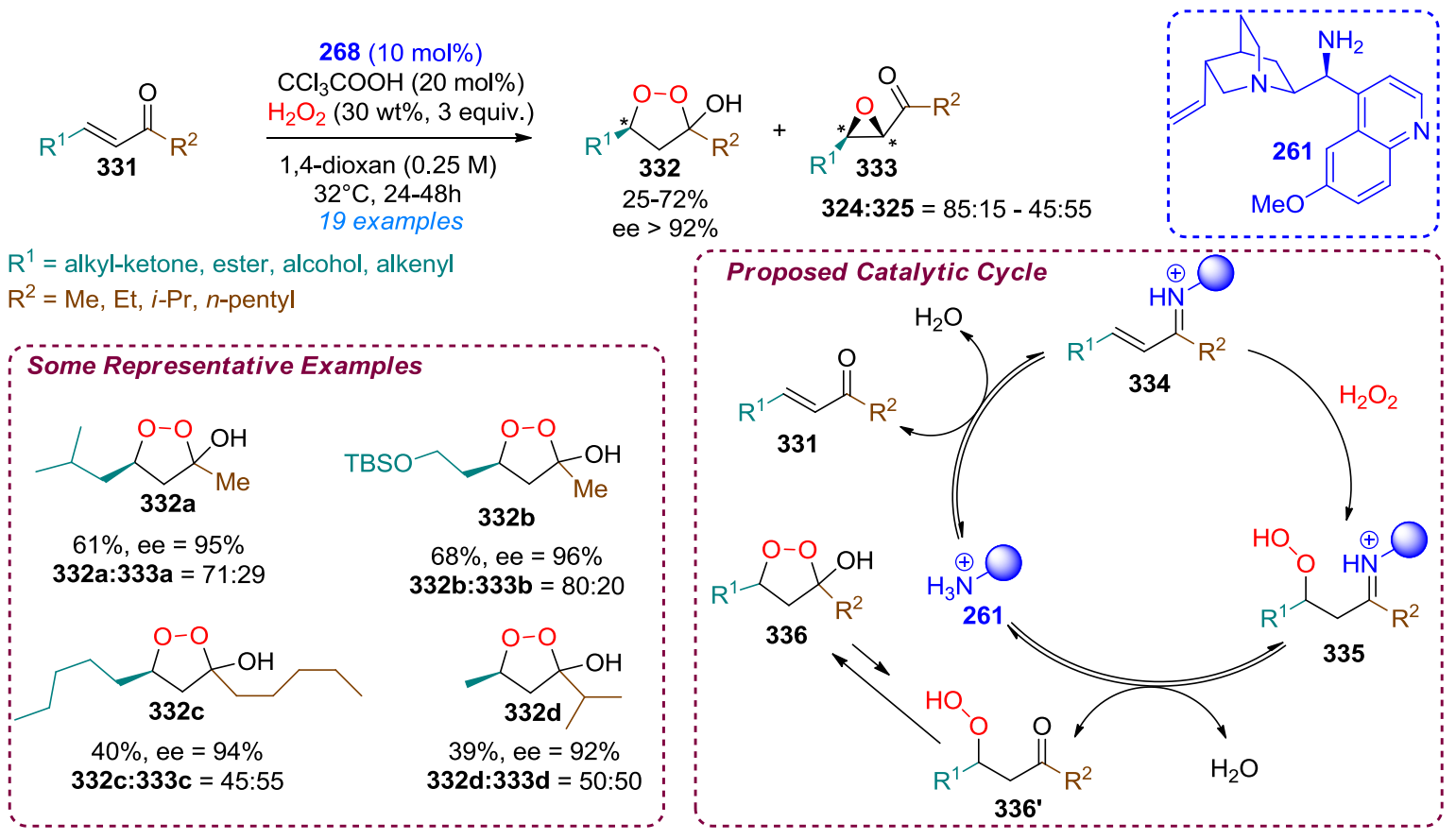

\section{Scheme 52}

\subsubsection{Addition to the carbonyl group}

Hydrogen peroxide is a very good nucleophile towards carbonyl group, and by consequence, its addition onto 1,3- and 1,4 diketones system was early studied in the 60's. ${ }^{206,207}$ Depending the stoichiometry of $\mathrm{H}_{2} \mathrm{O}_{2}$, adducts 336a-c and 337a-c could be obtained from acetylacetone or acetonylacetone. Notably, the use of two equivalents lead to formation of dimeric or polymeric products (Scheme 53). 
This method was also applied to the synthesis of tricycle $\mathbf{3 3 8}$, for the research of new anti-malarial drugs. ${ }^{208}$ Dussault and coworkers were able to cyclize compounds 340a-b under acid catalysis, which provided spiro-products 341a and 341b after in situ silyl deprotection, (Scheme 53). ${ }^{169}$
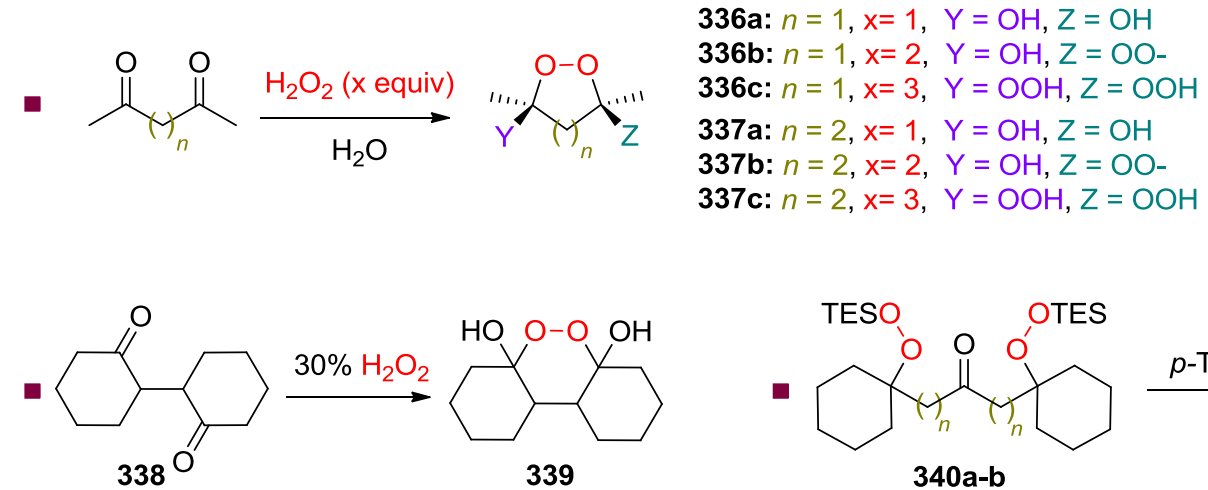

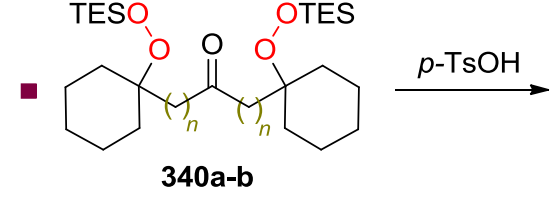

340a-b

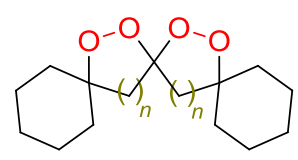

341a: $n=1,81 \%$ 341b: $n=2,88 \%$

\section{Scheme 53}

Terent'ev developed an original methodology from 1,3-diketones, by using some iodine and $\mathrm{H}_{2} \mathrm{O}_{2}$ to promote an iodoperoxidation from an internal olefin. ${ }^{209}$ Thus compounds 342a-e were transformed into 1,2-dioxolanes 343a-e, whereas diene such as 342f was transformed into original endoperoxide $343 \mathrm{f}$ through a double iodo-cyclization. The mechanism can evolve between two pathways: in path A, an iodocyclization from ketone group takes place, which produces oxycarbenium species 344a-e and finally 343a-e after $\mathrm{H}_{2} \mathrm{O}_{2}$ addition, while path $\mathrm{B}$ describes an inversion of the steps. A mechanical study would be necessary to determine the most plausible route, in particular by using a sequential addition of the reagents in one direction or the other and by analyzing the possible differences in the relative stereochemistry (Scheme 54). 


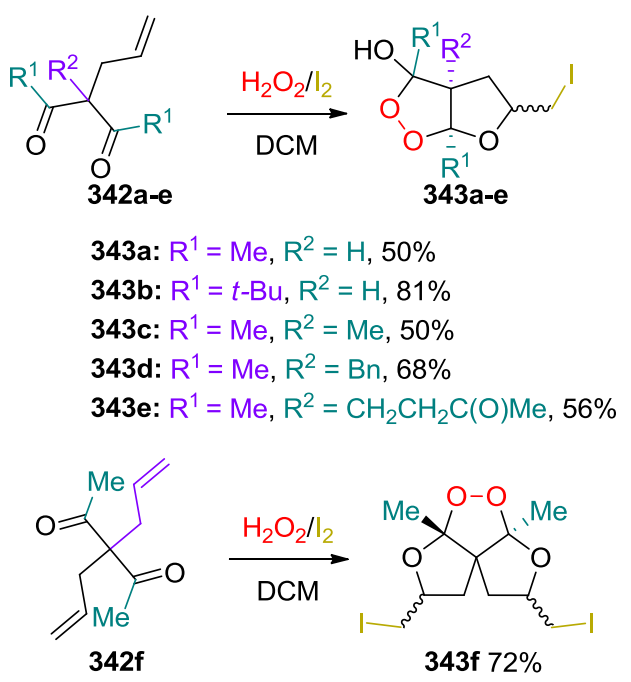

$343 f 72 \%$

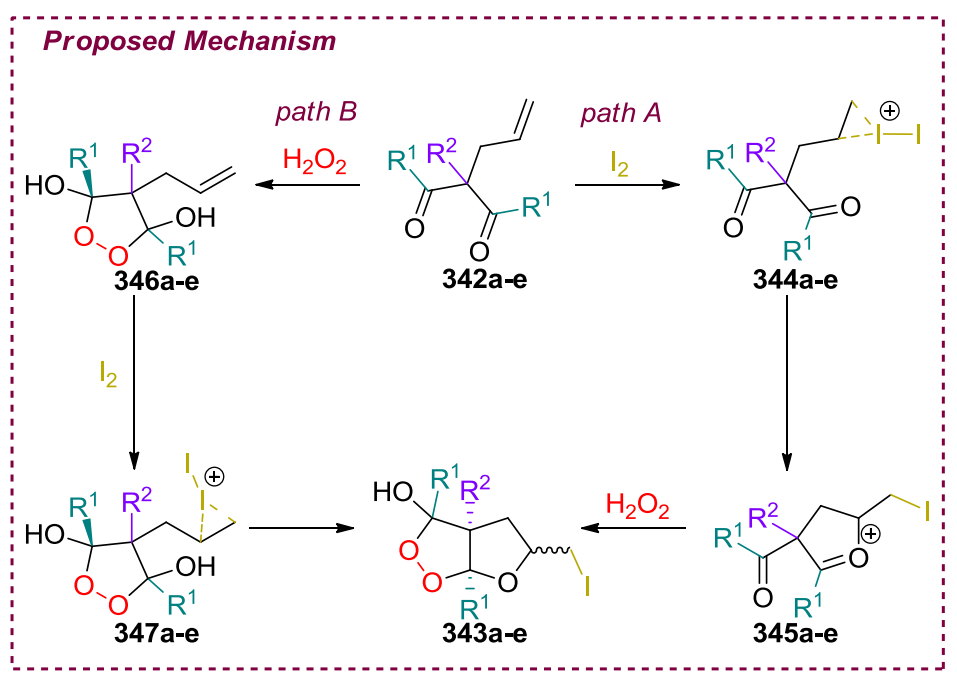

Scheme 54

An interesting study by Woerpel and coworkers concerns the addition of hydrogen peroxide to 1,3-keto-epoxides 348, which afforded trans-349 and cis-349 in a ratio of about 70:30.210 Aromatic substituents generally gave modest yields, whereas alkyl type substituents conducted the best conversions. Mechanistically, the first reacting function is difficult to determine between ketone and epoxide, but the most interesting feature in this transformation is probably the fact that the reaction first leads to the formation of the kinetic products 349, while a prolonged reaction time mainly leads to the formation of dioxolanes cis/trans-350, the thermodynamic products. The formation of peroxycarbenium species $\mathbf{3 5 1}$ is crucial for the formation of both kinetic and thermodynamic products, so addition of hydroperoxide to the carbonyl group is obviously the critical step in this reaction to afford 1,2-dioxolanes 350. (Scheme 55)

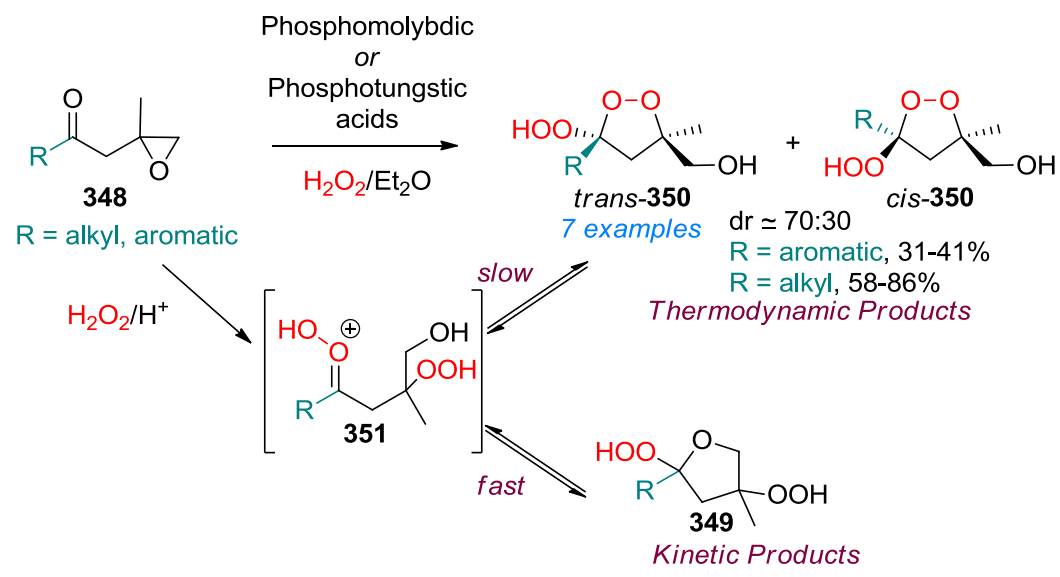

\section{Scheme 55}


Gemma, Campiani and coworkers transposed their study toward the ring opening of epoxides with hydroperoxide, ${ }^{175,176}$ to carbonyl derivatives. Therefore, after Isayama-Mukaiyama hydroperoxidation of olefins 352a-r, lactone 353a-r were reduced with DIBAL and resulting lactols 354a-r underwent a cyclization onto oxycarbenium species 355a-r to furnish 18 examples of 1,2-dioxanes, fused with a THF ring. ${ }^{211}$ Some amino derivatives were also recently prepared from 356f, using reductive amination, but the yields were low presumably to some competitive Kornblum-DeLaMare rearrangement. ${ }^{212}$ (Scheme 56)

The process was further applied to tetrahydropyranyl compounds. Thus, after hydroperoxidation of olefin 357a-c, hydroperoxycylization was performed under the same conditions to obtain intricate dioxanes 360a-c. ${ }^{213}$ (Scheme 56)

Application of the two above strategies to ketone derivatives $\mathbf{3 6 1 a - a c}$ and 363a-c afforded in presence of various alcohols, more than 30 ketals derivatives such as 362a-c or 364a. ${ }^{214}$ (Scheme 57 )

All these products prepared by the team of Gemma and Campiani were tested on Plasmodium $f$. parasites. However, the antimalarial activity was in general modest compared to drugs currently used to treat this disease (Schemes 56\&57).

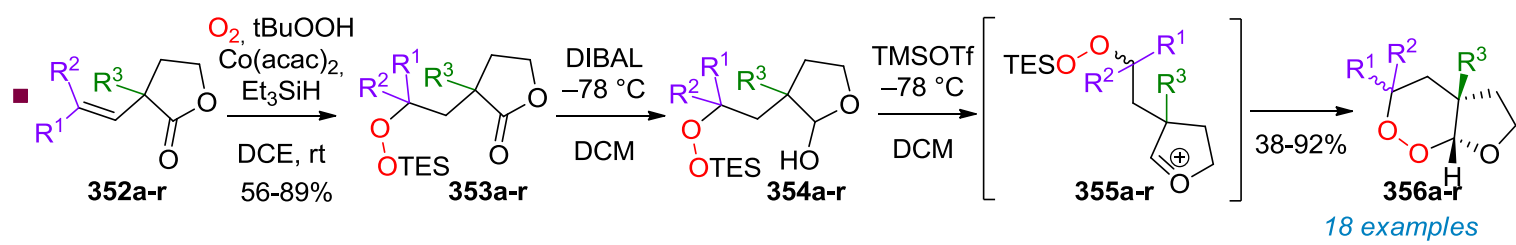



$356 a$

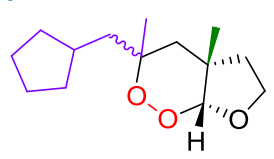

$356 \mathrm{~b}$

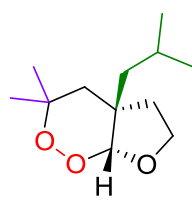

$356 c$

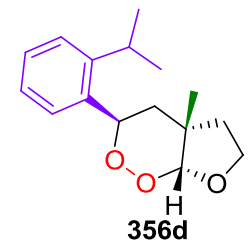

356d

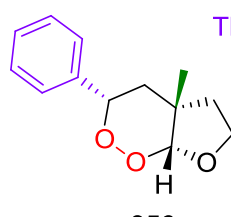

356 e
TBDPSO

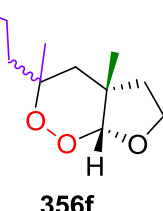

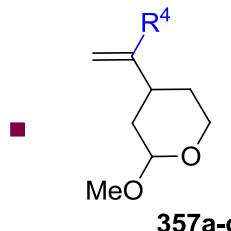
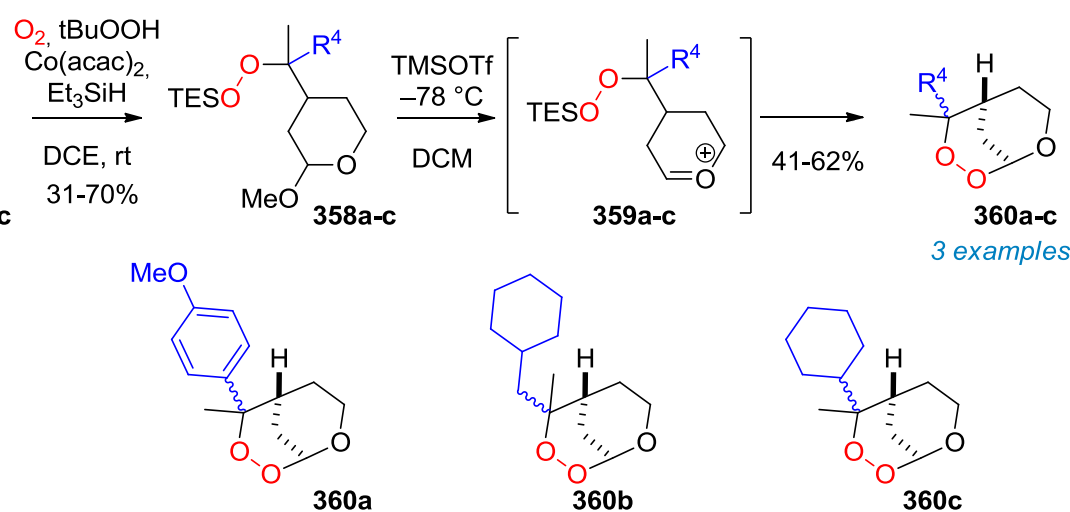

3 examples
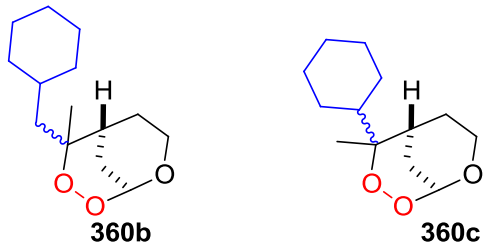


\section{Scheme 56}
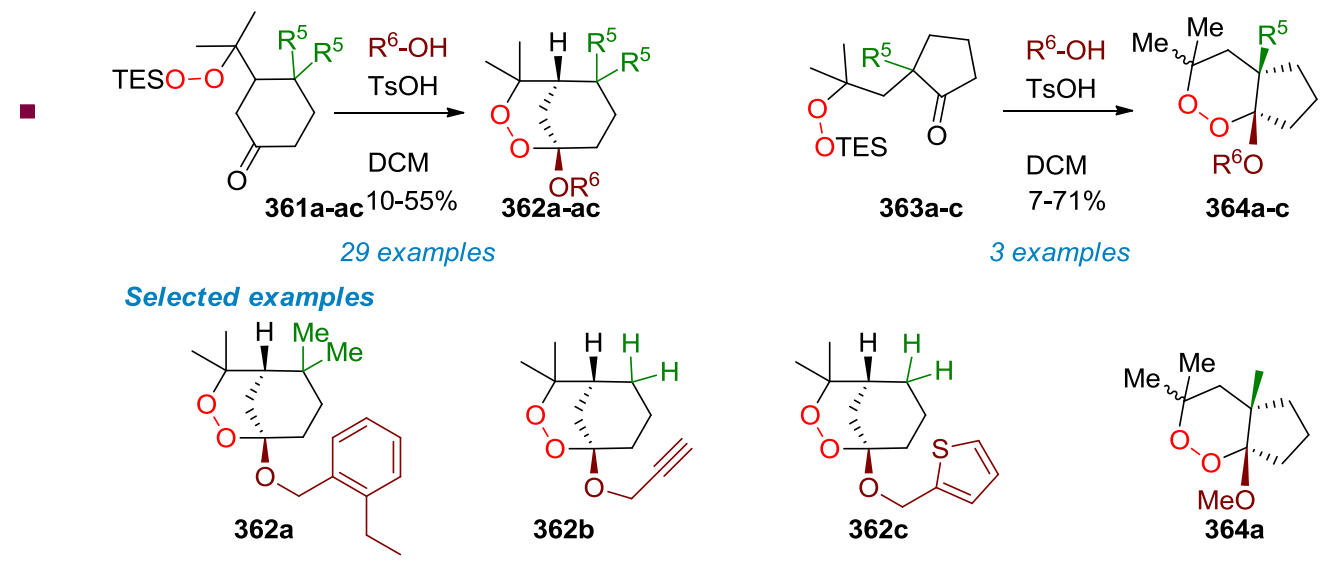

Scheme 57

\subsection{Peroxycylization involving Electrophilic Singlet Oxygen}

Singlet oxygen is an instable and very reactive oxygen species with a short lifetime, prepared in situ from triplet oxygen with a photo-sensitizer under light activation. Peroxides and endoperoxides can be obtained with this reagent from olefins, generally through two sort of processes: an ene-reaction or a [4+2] cycloaddition. Whereas ene-reaction can be only applied to mono-olefins, [4+2] cycloaddition are specifically applicable to dienes or higher conjugated systems. However, in this last scenario, the two processes can be in competition, depending the substrate, which leads to the formation of a hydroperoxide instead of a 1,2dioxene.

\subsubsection{Ene reaction}

Ene reaction with oxygen singlet consists generally in the preparation of hydroperoxides; however, in the presence of conjugated ketones, this reaction rises to the formation of hemi-endoperoxiketals. Specifically, Snider and coworkers studied this reaction for the synthesis of some natural products. Therefore, racemic chondrillin and plakorin were synthesized from conjugated ketone $\mathbf{3 6 5}$ by reaction with singlet oxygen, generated with the help of a photo-sensitizer under light irradiation, in a DCM:MeOH mixture. ${ }^{215}$ Transketalization of $\mathbf{3 6 6}$ with $\mathrm{MeOH}$ drove to a separable mixture of chondrillin and plakorin. Light irradiation presumably enolizes the conjugated ketone which can then react with singlet oxygen to form after 
elimination hydroperoxide 369. Further UV-mediated isomerization leads 369 finally to $\mathbf{3 7 0}$, which prefers to adopt an endoperoxide form. If the enolization of $\mathbf{3 6 5}$ seems obvious, Addition of singlet oxygen could react from species $\mathbf{3 6 7}$ through an ene reaction or a [4+2] cycloaddition process. However, since hydroperoxide $\mathbf{3 6 9}$ has been isolated and identified as a by-product in this transformation, the ene reaction is probably the most plausible mechanistic pathway. A very similar strategy was adopted by Dussault in an enantioselective synthesis of chondrillin and plakorin. In particular, they used a light mediated isomerization a double bond from an intermediate such as $\mathbf{3 6 9}$ (Scheme 58). ${ }^{216}$

Ene reaction with singlet oxygen was also applied by Snider and coworkers to the total synthesis of acetylsaturejone and isoacetylsaturejone. ${ }^{217}$ Photooxygenation of intermediate $\mathbf{3 7 1}$ with rose Bengal afforded acetylsaturejone through a direct ene reaction with oxygen triplet. In contrast photooxygenation with $\mathrm{CuSO}_{4}$ as a sensitizer afforded isoacetylsaturejone through formation of enol 372. Differences observed in these two conditions are not clearly understood. However, the ene reaction is probably the biomimetic pathway towards the production of these last natural products (Scheme 58).

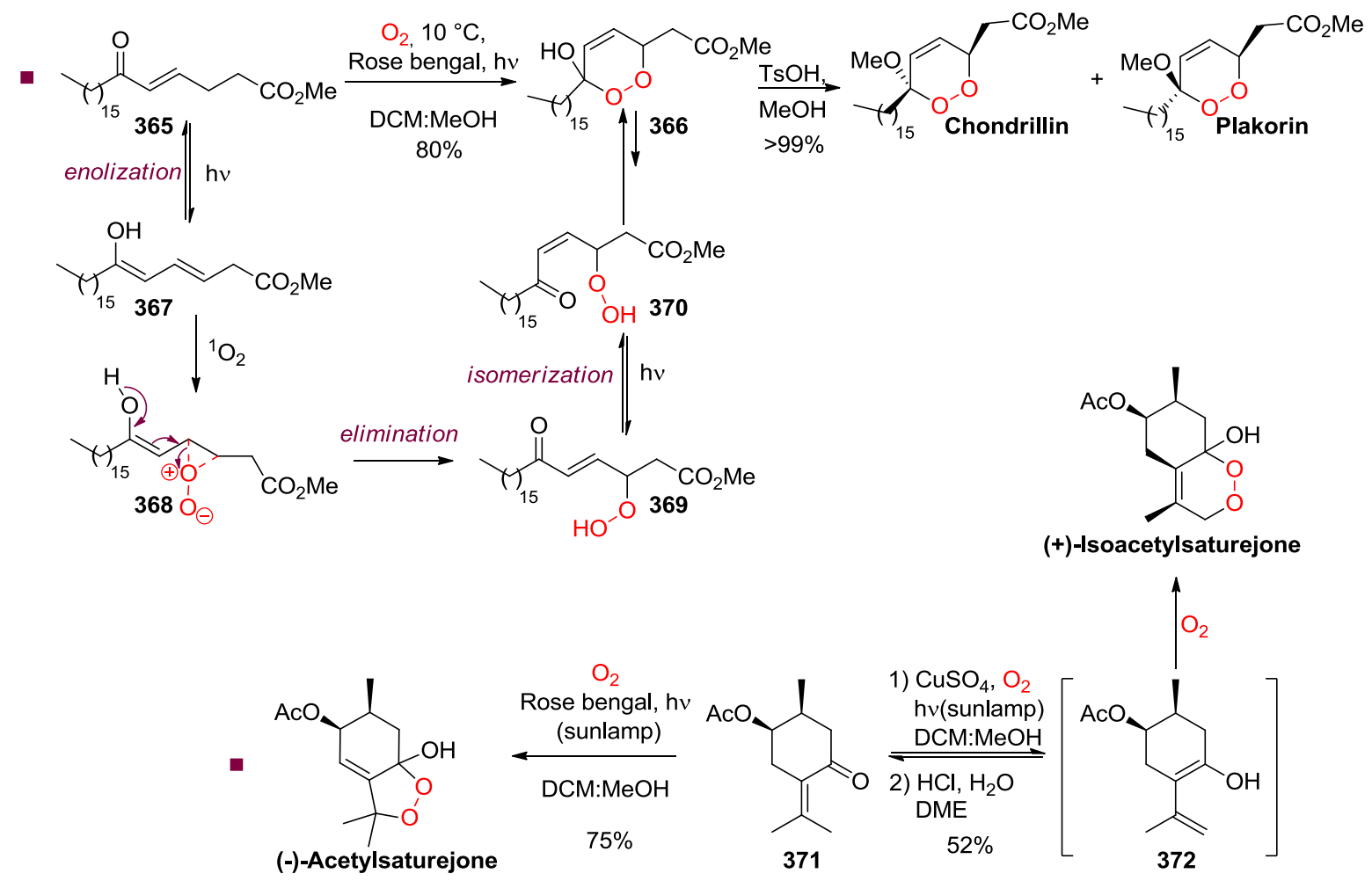

Scheme 58 
The ene reaction was also utilized more recently in the synthesis of the diterpene (+)-Jungermatrobrunin A by Lei, X. and coworkers, where the final step was the peroxidation of $\mathbf{3 7 3}$ with singlet oxygen. ${ }^{218}$ Extensive screening of reaction conditions appointed that rose Bengal as sensitizer in MeCN/pyridine (40:1) using a $24 \mathrm{~W}$ white LED during $36 \mathrm{~h}$ gave the best results for this transformation, giving (+)Jungermatrobrunin A in $57 \%$ yield (65\% conversion). Light wavelength was very important since UV promoted the rearrangement to product 374 (Scheme 59).

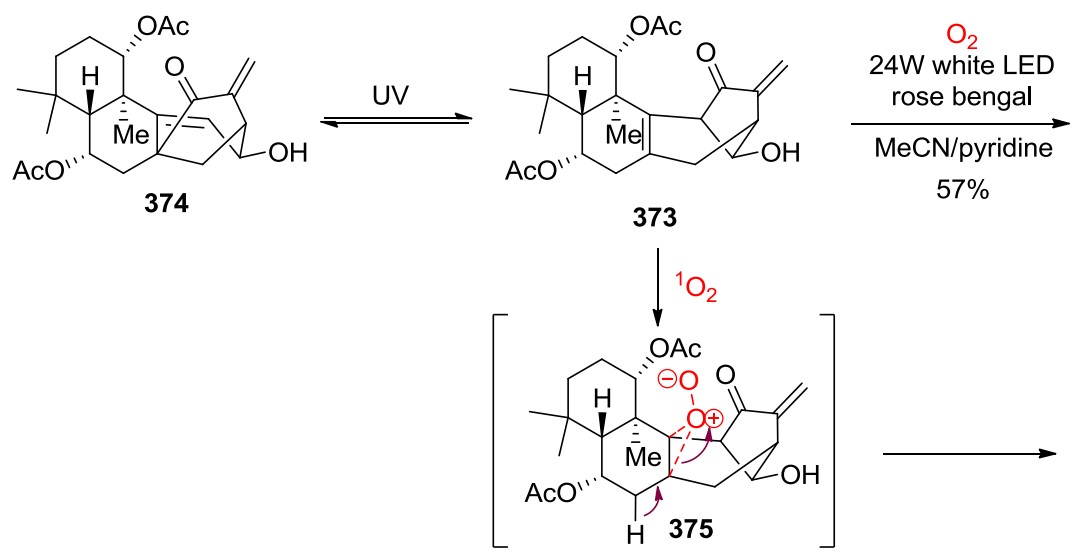

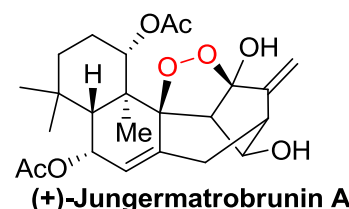

(+)-Jungermatrobrunin A

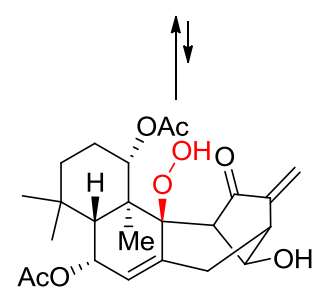

\section{Scheme 59}

In a very specific example towards photooxygenation of some allylstannes, Dussault and coworkers demonstrated that singlet oxygen is able to induce directly the formation of 3,5-disubstituted dioxolane rings from functionalized allyl stannanes through a rearrangement including a 1,2-migration of the $\mathrm{SnBu}_{3}$ group. ${ }^{219}$ Thus allylstannanes 376a-c were transformed in expected hydroperoxides 377a-c through a classical ene-reaction or in dioxolanes 378a-c via a migration of $\mathrm{SnBu}_{3}$ onto reactional intermediate 379a-c, evolving into species 380a-c, which lead to the endoperoxide. The scope of the reaction is limited to few examples. Only $\mathbf{3 7 8 b}$ was obtained as a sole reaction product, while $\mathbf{3 7 8 a}$ or $\mathbf{3 7 8 c}$ were obtained with less selectivity towards hydroperoxides $377 \mathbf{a}$ or $377 \mathbf{c}$. The transformation seems stereospecific, but is pretty difficult to predict. Nevertheless, the nature of the " $\mathrm{X}$ " group, the stereochemistry of the double bond and the solvent seem crucial to the selectivity between $\mathbf{3 7 7}$ and $\mathbf{3 7 8}$ (Scheme 60). 

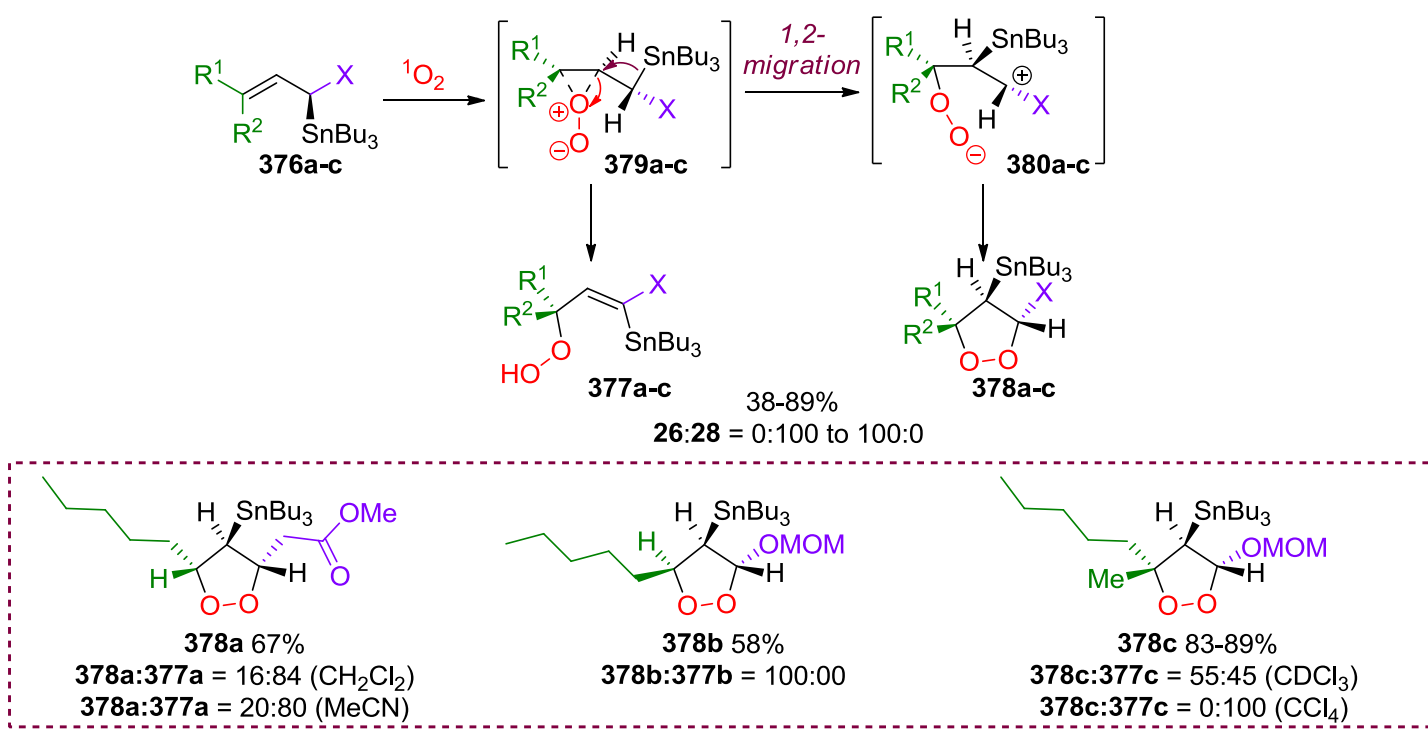

Scheme 60

\subsection{2 [4+2] Cycloaddition}

The [4+2] cycloaddition of singlet oxygen with dienic systems is probably the most famous transformation for synthesizing endoperoxides, with approximately 1500 occurrences in the literature via a search with Scifinder ${ }^{\circledR}$. The peroxidation of ergosterol was early described by Windaus and Brunken in the 20's by using similar conditions what we use today, i.e. an oxygen source, a filament lamp to produce photons and a photosensitizer such as eosin to convert triplet oxygen in singlet oxygen. ${ }^{220}$ Because of the high number of occurrences, a selection of research reports will be here presented, focusing on most recent and/or most noteworthy examples.

As explained in the introduction of paragraph 3.4, [4+2] cycloaddition with singlet oxygen can in competition with ene-reaction. The outcome mainly depends on the structure of the substrate, thus Harwood and coworkers principally studied this phenomenon towards the total synthesis of mycaperoxide B. ${ }^{221}$ Indeed, they hypothesized that mycaperoxide $\mathrm{B}$ is probably produced biosynthetically through [4+2] cycloaddition followed by reduction of the olefin contained in the 1,2-dioxene. However, their study could not validate their proposed biomimetic pathway, since diene 381a underwent a ene-reaction giving 382a (for both $Z: E$ or $E$ : $E$ isomers), in contrast to substrate $\mathbf{3 8 1 b}$ (non-methylated olefin), which underwent a [4+2] cycloaddition giving 1,2-dioxene $\mathbf{3 8 3} \mathbf{b}$. (Scheme 61) 


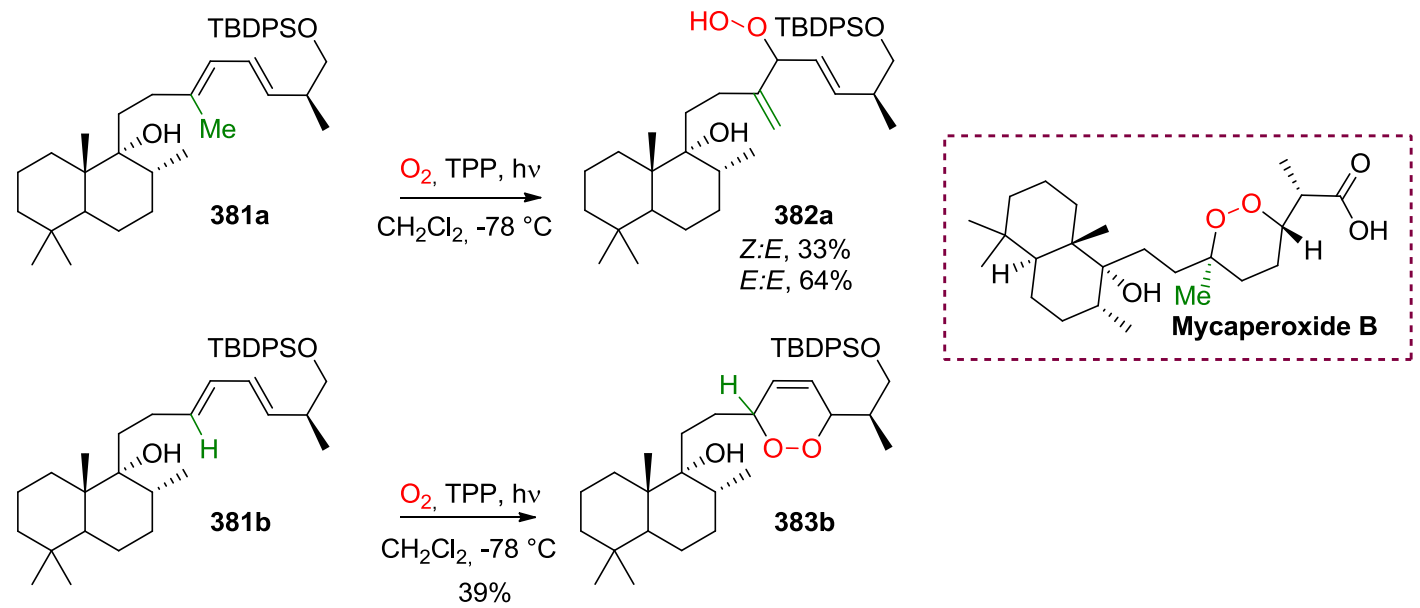

\section{Scheme 61}

The [4+2] cycloaddition was nevertheless used successfully in the total synthesis of some natural endoperoxides. Steliou and Yao presented a study of total syntheses of unnamed natural products belonging to the plakortide family (see paragraph 2.1.2), where the final key step was a [4+2] cycloaddition with singlet oxygen. Thus, diene $\mathbf{3 8 4}$ underwent a cycloaddition to produce 1,2-dioxenes $\mathbf{3 8 5 a}$ and $\mathbf{3 8 5} \mathbf{b}$ as a mixture of diastereomers (Scheme 62). ${ }^{222}$

Jung and coworkers also used a [4+2] cycloaddition during the synthesis of 6epi-plakortolide E. A cycloaddition reaction from diene $\mathbf{3 8 6}$ afforded dioxenes $\mathbf{3 8 7 a}$ and $\mathbf{3 8 7} \mathbf{b}$ in the same ratio than the stereoisomeric purity of the starting material. Diastereomer $\mathbf{3 8 7 a}$ was then transformed in 4 steps into 6-epi-plakortolide E, notably by applying an iodolactonisation followed by a radical reduction of the remaining iodide (Scheme 62). ${ }^{223}$ 

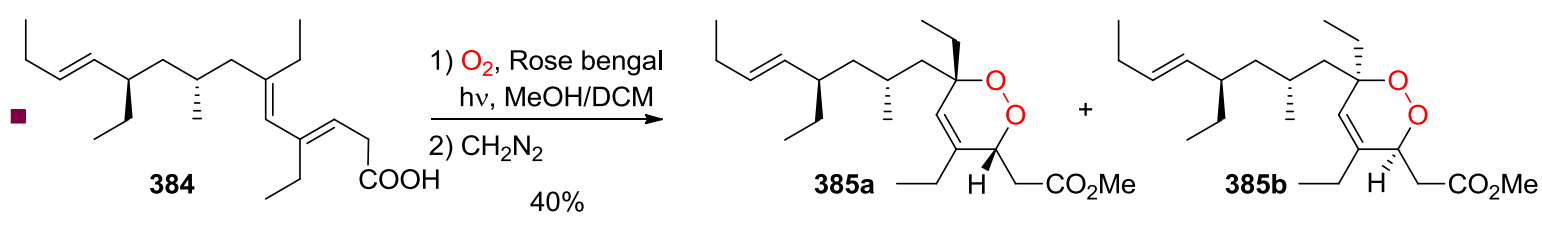

$\mathrm{dr}=2: 1$
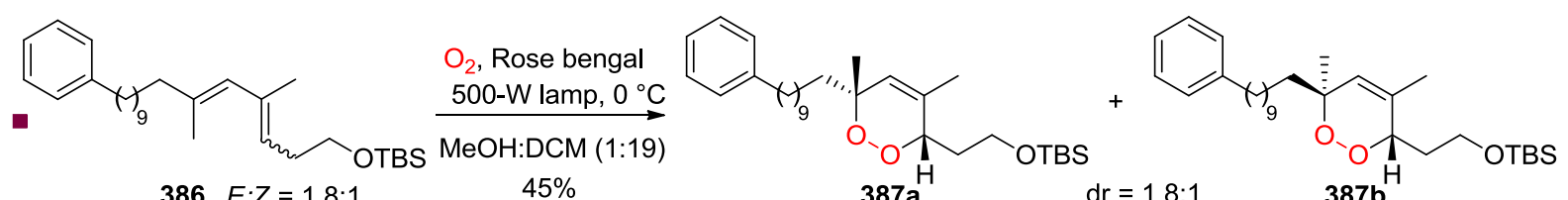
$387 \mathrm{a}^{\mathrm{H}} \quad \mathrm{dr}=1.8: 1$ $387 b^{H}$

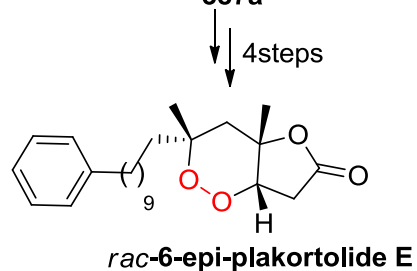

Scheme 62

Whereas 1,2-dioxenes can be kept as a final objective because the target needs the unsaturation inside the endoperoxide, there are some noteworthy examples where the 1,2-dioxene could be converted into a 1,2-dioxane. Because metal catalyzed hydrogenation often lead to homolytic reduction of peroxide bond, the utilization of diimide seems obviously the reagent of choice for this transformation. Thus, guianolide derivative $\mathbf{3 8 8}$ underwent a cycloaddition reaction to make thaperoxide, a synthetic anti-malarial agent, and a dimide reduction gave access to 1,2-dioxane 389. ${ }^{224}$ Many endoperoxide analogs of $\mathbf{3 8 9}$ were then prepared (Scheme 63 ).

Another example of a selective 1,2-dioxene reduction is represented by the synthesis of gracilioether $\mathrm{F}$ by Wong and coworkers. ${ }^{225}$ Application of a [4+2] cycloaddition on $\mathbf{3 9 0}$ with singlet oxygen, followed by a diimide reduction gave rise to dioxane 391. The endoperoxide was then reduced and the primary alcohol oxidized to give the natural product (Scheme 63). 


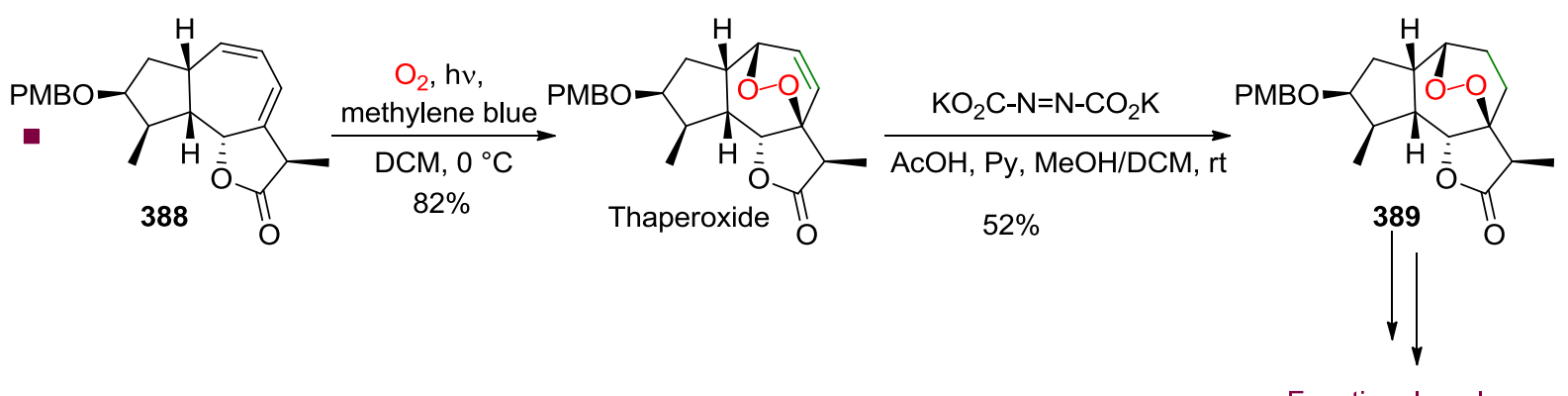

Functional analogs
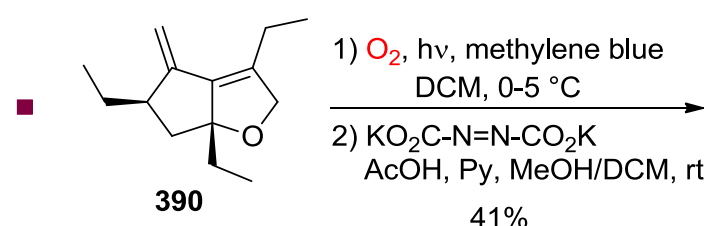

$41 \%$
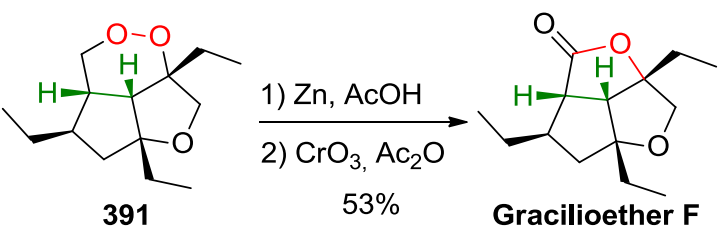

Scheme 63

Such as with the past example depicted in Scheme 57, 1,2-dioxenes are more often used as intermediates to prepare other specific functionalities. Nicolaou and coworkers used the 1,2-dioxenes present in $\mathbf{3 9 3}$ to perform a double KornblumDeLaMare rearrangement, producing conjugated 1,4-hydroxyketones, which were further hydrogenated to give Epicoccin (Scheme 64).226,227

In a study towards mulinane diterpenoids, Xie and coworkers prepared mulinic acid by [4+2] cycloaddition from diene 394. Reaction of mulinic acid with a ruthenium complex gave rise to the formation of isomulinic acid (Scheme 64).228

Another interesting example transformed 1,2-dioxenes into furans under Appel reaction conditions. Thus, diene 395 underwent first a cycloaddition with singlet oxygen to form 1,2-dioxene 396 and further reaction with $\mathrm{PPh}_{3}$ and $\mathrm{CBr}_{4}$ led to 397 in good yield. ${ }^{229}$ The tribromomethylanion degrades the peroxide into a hydroxyketone through a Kornblum-deLaMare rearrangement and a dehydration by reaction of the ketal with the bromotriphenylphosphonium cation leads to the furan. Further functionalization through an olefin cross-metathesis reaction gave $\mathrm{F}_{5}$ methyl ester (Scheme 64). 

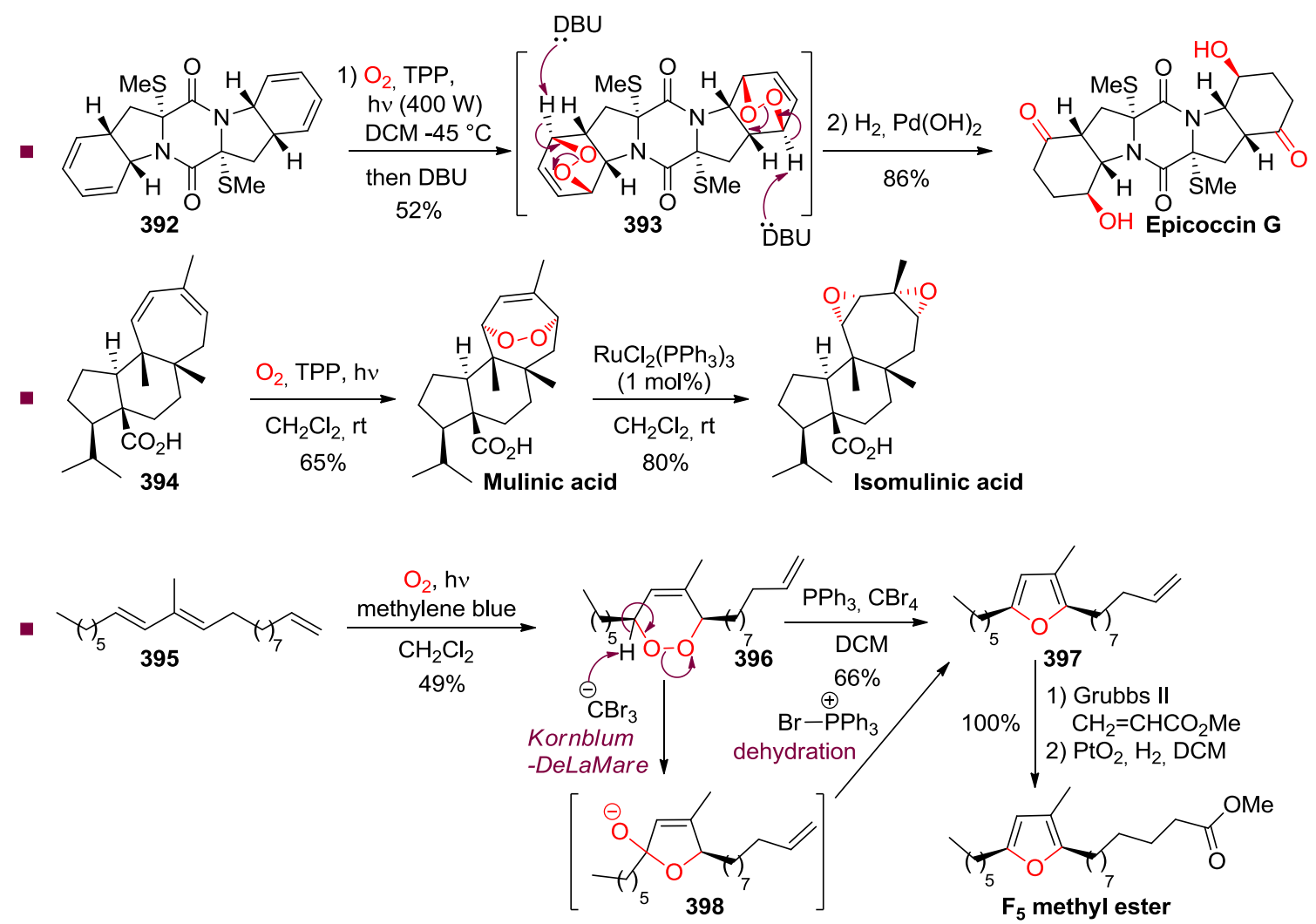

\section{Scheme 64}

Another noteworthy example concerns a work towards the total synthesis of Okundoperoxide, where the 1,2-dioxene was converted into another endoperoxide. ${ }^{230}$ Diene 399 underwent first a [4+2] cycloaddition with oxygen singlet giving dioxene 400. A transformation of the diol in allylic epoxide $\mathbf{4 0 1}$ allowed then to perform the last key step, involving the silyl deprotection triggering with the endoperoxide ring opening and the final cyclization onto the olefin through a Brønsted acid activation of the oxirane (Scheme 65).

Cabrera and coworkers performed a similar transformation onto tetracyclic phenol 403 to produce endoperoxide 408. ${ }^{231}$ First, a [4+2] cycloaddition reaction with singlet oxygen takes place, and hydroperoxy group is then released upon elimination by the geminal hydroxyl group. Intermediate 405 is then subjected to a supplementary formal peroxycyclization with singlet oxygen affording endoperoxide 408. During this last sequence, the reaction seems to not pass exactly through a [4+2] cycloaddition, following author's hypotheses. (Scheme 65) 

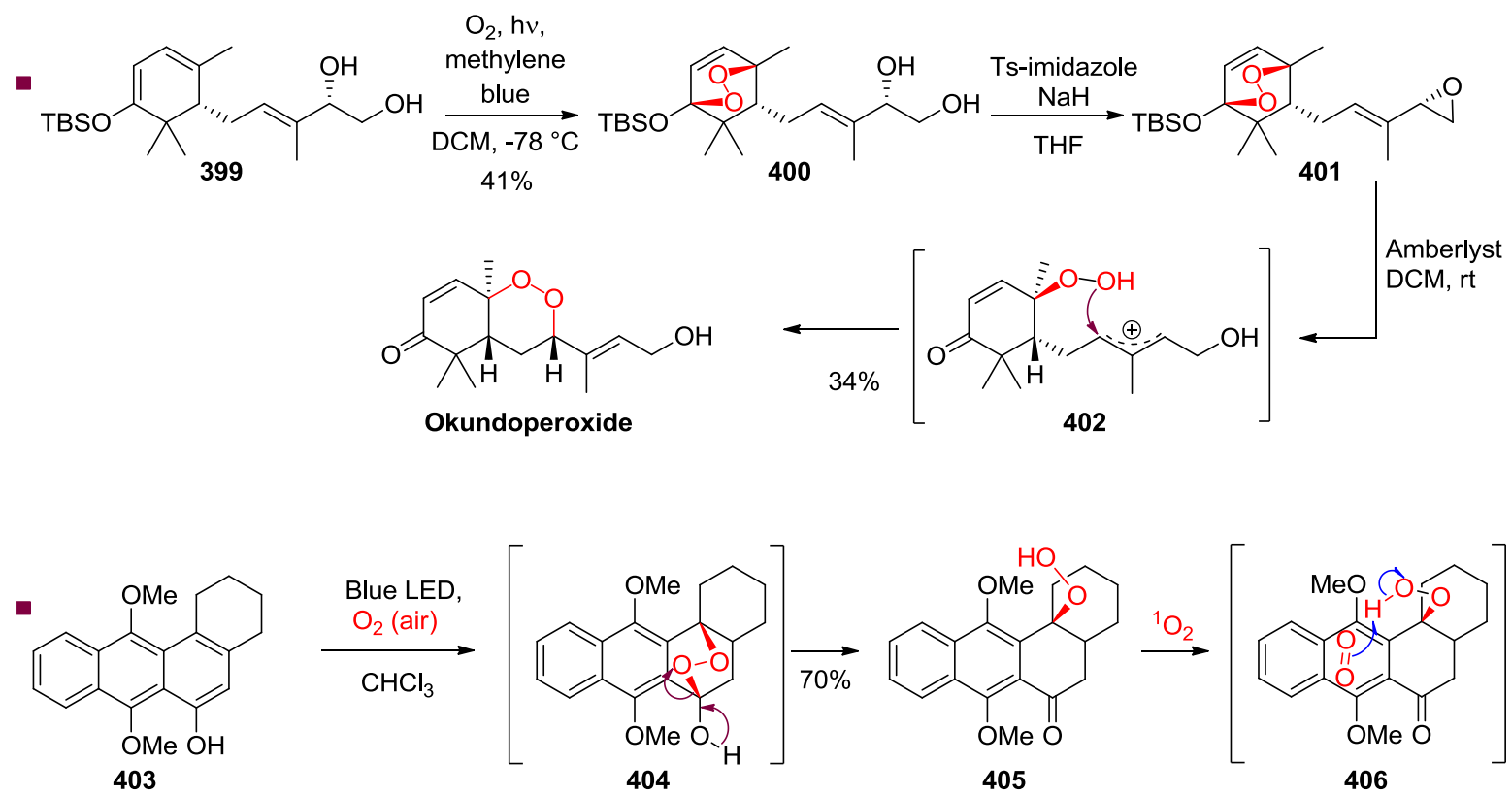

404

405

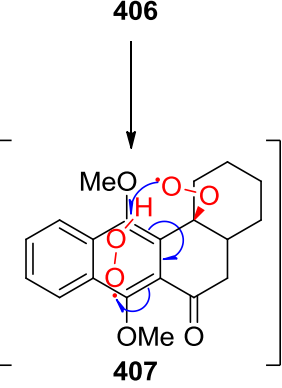

\section{Scheme 65}

\subsection{Peroxycylization and Functionalization of Endoperoxyketals} through the Generation of Peroxycarbenium Species.

We saw in paragraph 3.3.3 that carbonyl groups are ideal substrates to prepare some peroxyketals through the creation of a new $\mathrm{C}-\mathrm{O}$ bond. Peroxyketals, through the formation of reactive peroxycarbenium species are interesting precursors to build new endoperoxides via the formation of a new $\mathrm{C}-\mathrm{C}$ bond. In particular, two main processes will be described in this section: 1) Formal [3+2] cycloaddition between peroxycarbenium species and olefins, allowing the formation of 1,2-dioxolanes. 2) Functionalization of endoperoxyketals, providing an excellent route to many functionalized 1,2-dioxanes or 1,2-dioxolanes.

\subsubsection{Formal [3+2] cycloadditions}

Seminal work of Nojima and coworkers in the 80's demonstrated for the first time that some 1,2-dioxolanes could be obtained from ozonides (namely 1,2,4- 
trioxolanes) by reaction with some 1,1-disustituted olefins in presence of $\mathrm{BF}_{3} \cdot \mathrm{OEt}_{2 .}{ }^{232,233}$ Thus, ozonide 409, was for example, transformed into 1,2-dioxolane 410 by reaction with methylstyrene 91, releasing some benzophenone. The mechanism of the reaction is somehow similar to all the reactions that will be following in this section. An activation of the peroxyketal by the Lewis acid such as in $\mathbf{4 1 1}$ induces the fragmentation of the ozonide in acetophenone and peroxycarbenium 412. The ene-reaction of 91 onto peroxycarbenium species 412 takes then place to afford in a first step carbonium species $\mathbf{4 1 3}$ and in a second step 1,2-dioxolane $\mathbf{4 1 0}$. Because formation of $\mathbf{4 1 0}$ is sequential through species $\mathbf{4 1 3}$, the reaction cannot be considered like a concerted [3+2] cycloaddition but more like a formal process, which influences stereochemistry outcome of the reaction by giving a mixture of diastereomers (Scheme 66).



Scheme 66

Since ozonides possess an axial symmetry, Lewis acid mediated fragmentation of these heterocycles can provide in general two different peroxycarbenium species. Therefore, Dussault and coworkers were able some years later to apply this formal [3+2] cycloaddition on disubstituted ozonides such as with 414. ${ }^{234,235}$ In this case, a mixture of products $\mathbf{4 1 5}$ and $\mathbf{4 1 6}$ was obtained from the formation of peroxycarbenium species 417 and 418. Regioselectivity in the fragmentation of ozonides cannot always be controlled, which is a drawback with these substrates (Scheme 67). 


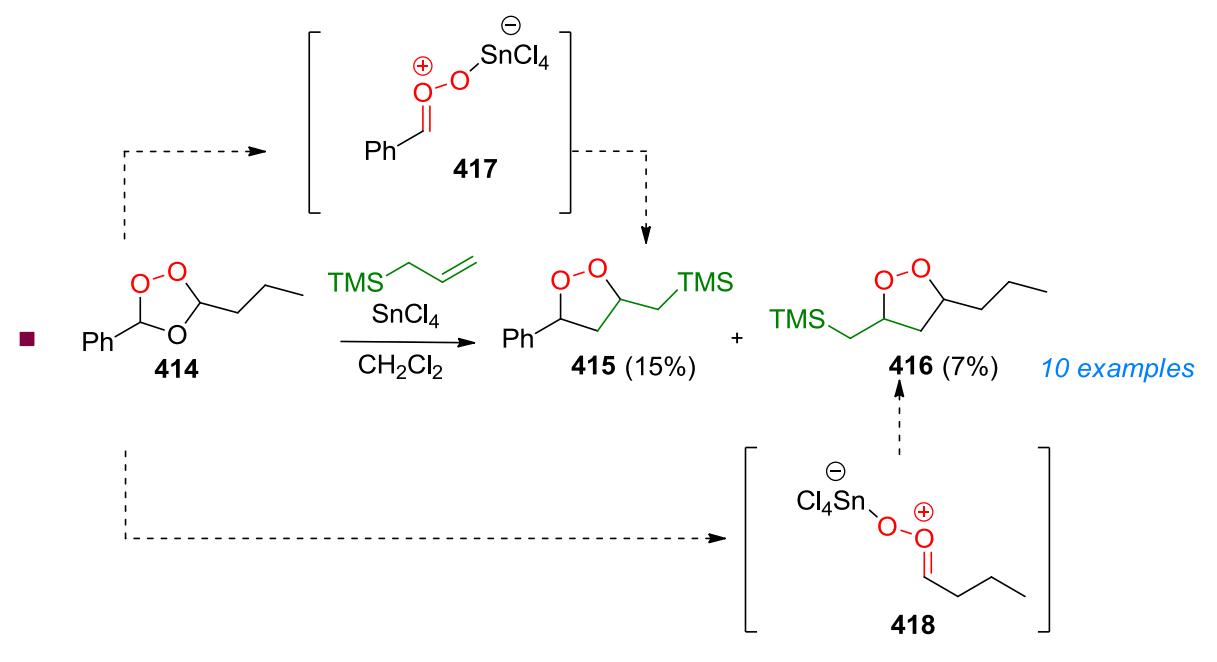

\section{Scheme 67}

A major improvement, in the formal [3+2] cycloaddition between olefins and peroxycarbenium species, was the utilization of acyclic hemiperoxyketals or hemiperoxyacetals as precursors to perform this transformation.236,237 Allylation of these species such as with allyltrimethylsilane, afforded directly 1,2-dioxanes. No regioselectivity issue is observed with the peroxyketals compared to ozonides, but some low yields in this reaction can be attributed to competition between alkoxy and hydroperoxyl group as nucleofuge. The reaction is working pretty well on ketal derivatives but the reaction is more difficult with peroxyacetals such as 419c. Using a methoxyethylether rather than a methoxy group as nucleofuge improves the yield because of its ability to chelate titanium or tin chloride. Thus compounds $420 a-d$ were prepared from alkoxyhydroperoxy ketals 419a-d, however no diasteroselective ratio was reported for these compounds meaning they were probably prepared as 1:1 mixture of diastereomers (Scheme 688).

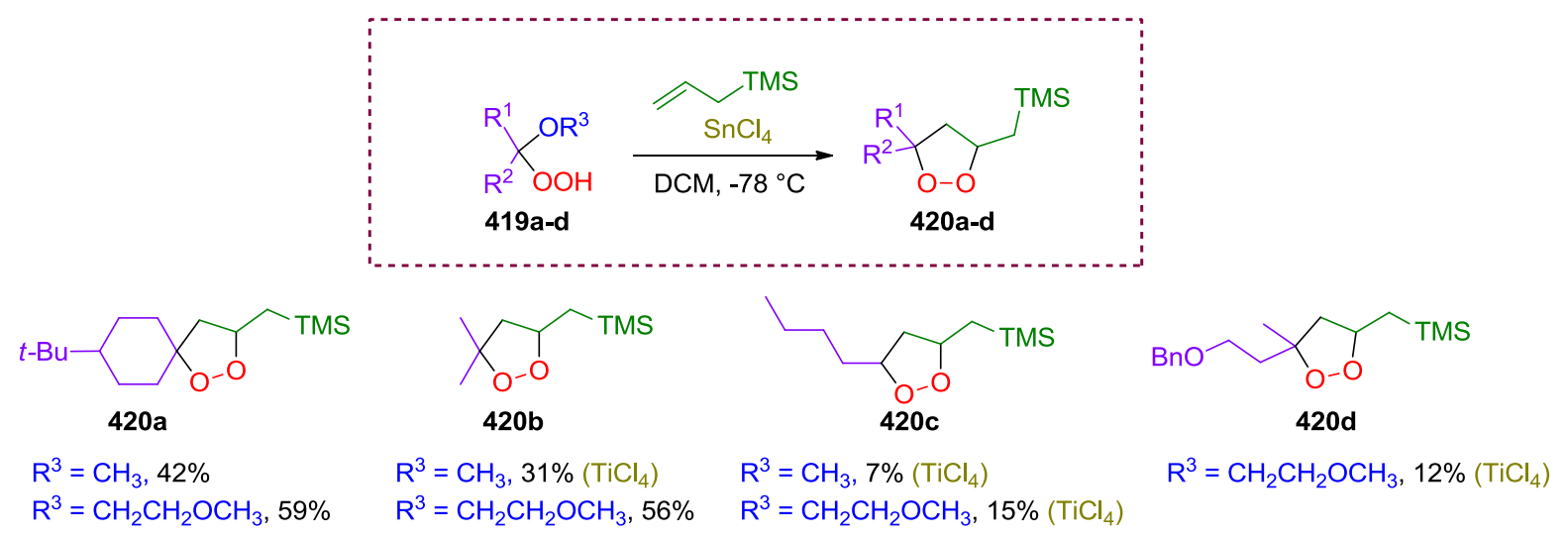

Scheme 68

Reaction was further studied and exemplified by Woerpel and coworkers on different peroxyketals. ${ }^{238,239}$ Their major input was firstly to use protected 
peroxyketals as silylether to promote the reaction. Triethylsilyl group is particularly suitable because protection of the hydroperoxide greatly improves the process during activation of the peroxyketal with Lewis acid, and this silyl group is sufficiently labile under reaction conditions to perform a spontaneous cyclization, rather than allowing an elimination to occur as in any Sakurai or ene-reaction. In a first study, authors used a disilylperoxyketals 421-423 to generate peroxycarbenium species (method A), ${ }^{238}$ while in a second study they used silyloxyperoxyketals 424-426 (method B). ${ }^{239}$ The use of method A or B is dependent of the substrates and both methods are complementary. Various 1,2-dioxolanes were then prepared (427-431) giving yields in the same range, whatever the starting material, prepared from both methods. (Scheme 69)

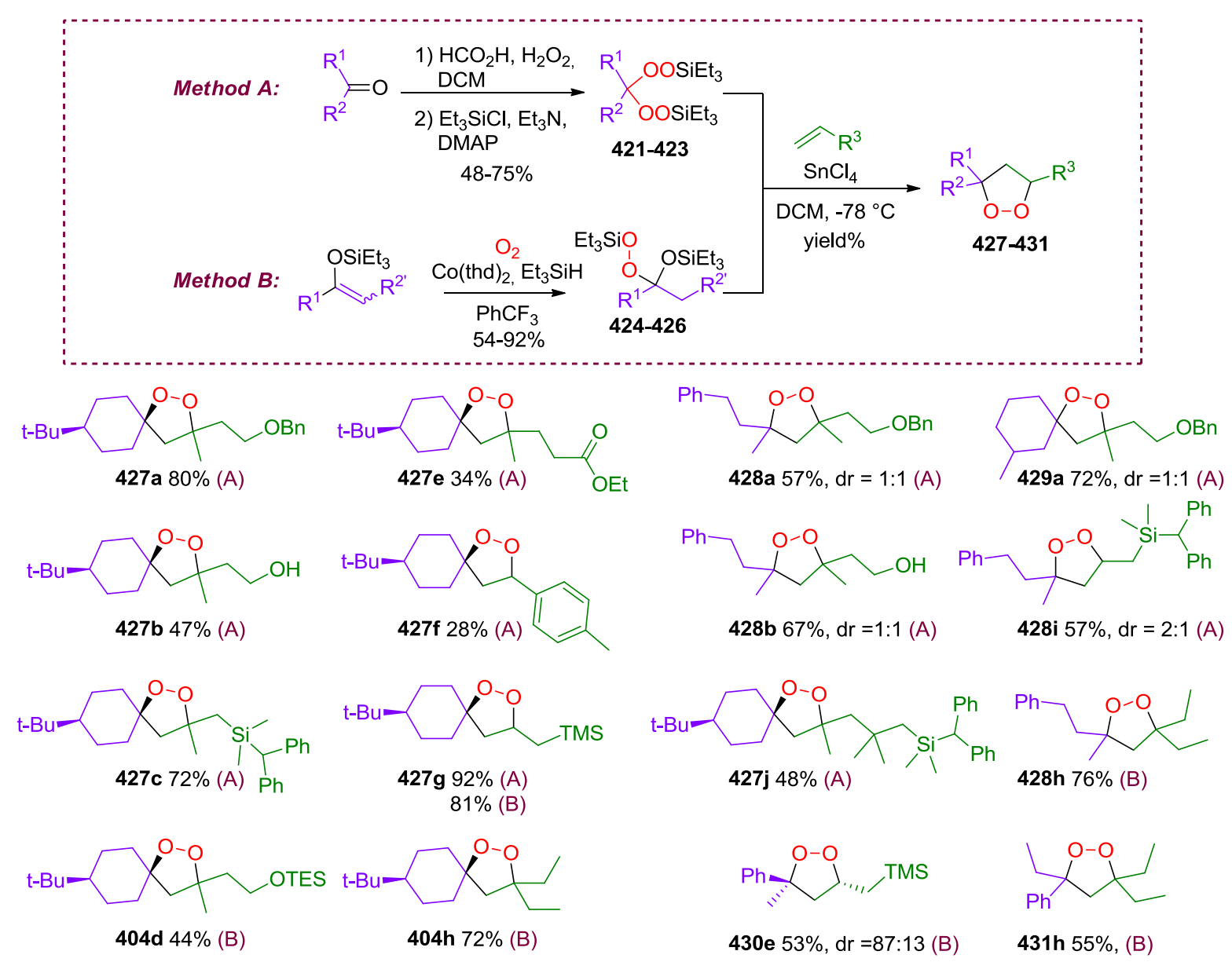

Scheme 69

Whereas the scope of the reaction was mainly limited to allylsilanes and 1,1disubstituted alkenes, the formal [3+2] cycloaddition with other type of olefins fails. Indeed, $\mathrm{Wu}$ and coworkers observed that expected reaction products rearranged into some side products presenting some alkyl group migration, instead of the expected 
1,2-dioxolane. ${ }^{240}$ This phenomenon was already observed by Nojima ${ }^{232}$ and later by us under strong Lewis acid conditions. ${ }^{241}$ Nevertheless, Wu and coworkers discovered that vinylether and vinylamine derivatives are convenient reagents for the [3+2] cycloaddition reactions with peroxycarbenium species. ${ }^{240}$ Thus, many examples with 2,3-dihydrofuran were performed giving several fused bicyclic compounds. The reaction worked also with $\mathrm{N}$-Boc-2,3-dihydropyrol and with acyclic derivatives to give for example 432e or 432f. This method allows more generally the introduction of an alkyl substituent in position 4 of the 1,2-dioxolane (Scheme 70).

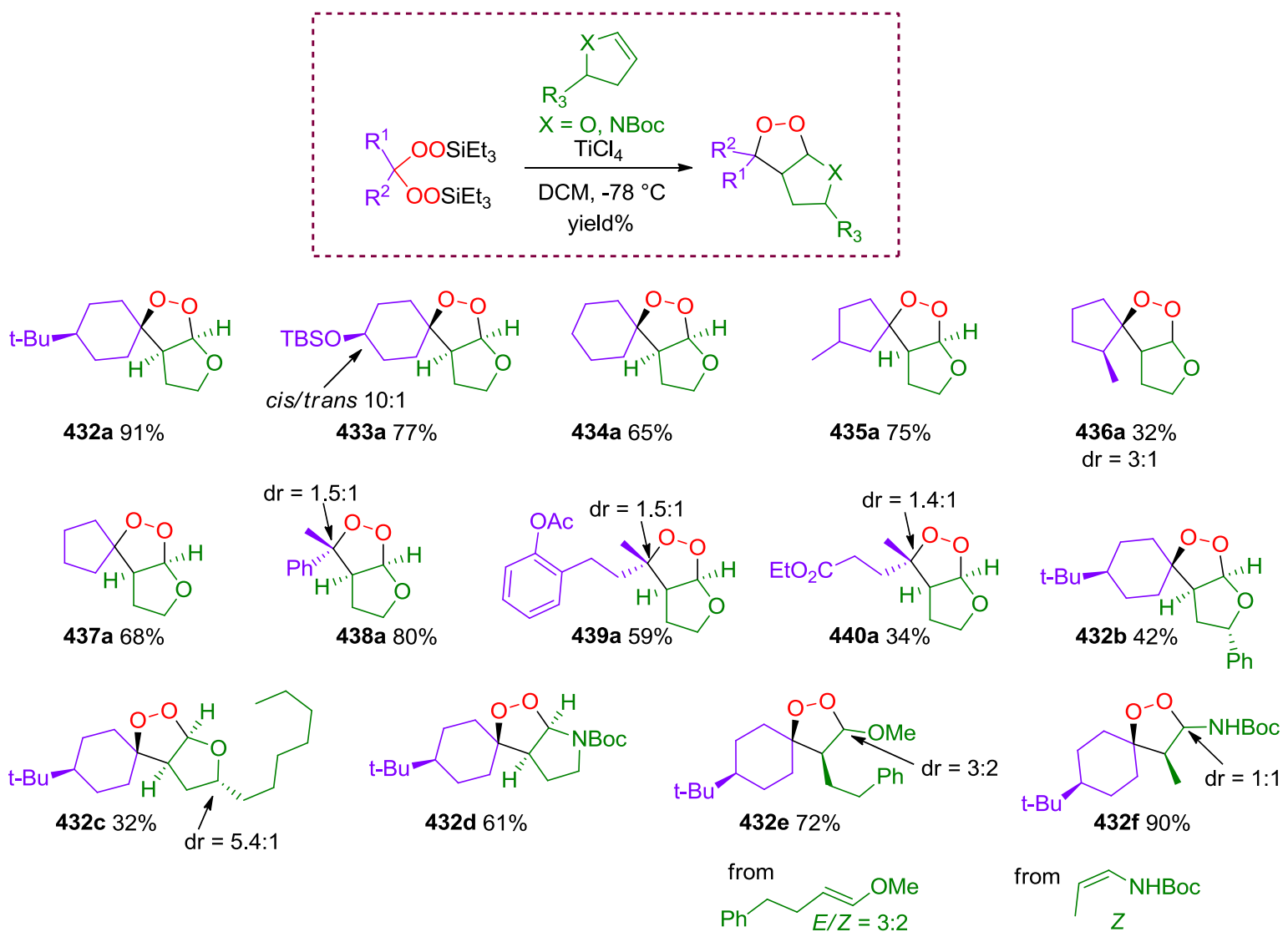

Scheme 70

The [3+2] cycloaddition was also experienced in an intramolecular fashion, like from compound 441, which was converted into a mixture of diastereomers $442 a$ and 442b, respectively in 22 and $64 \%$ yield. ${ }^{242}$ (Scheme 71)<smiles>C=C1c2ccccc2COC1CCC(C)(OCC)OOCC</smiles>

441

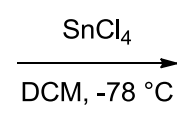<smiles>CC12CC[C@H]3OCc4ccccc4[C@]3(C1)OO2</smiles>

442a $22 \%$<smiles>CC12CCC(OOC3(C1)OCc1ccccc13)C2(C)C</smiles>

442b $64 \%$

\section{Scheme 71}


The formal [3+2] cycloaddition, using the conditions developed by Woerpel and coworkers, was then used in many application towards the search of new bioactives 1,2-dioxlanes. Thus, Vennerstrom and coworkers developed a library of new anti-malarial candidates exhibiting different endoperoxides, inspired from the synthetic antiplasmodal agent arterolane. They particularly replaced the 1,2,4trioxolane present in this bioactive compound by a 1,2-dioxolane such as in product 443. ${ }^{243,244,245}$ However the results were disappointing in term of antimalarial activity, compound 443 being more than 100-fold less active than arterolane against different strains of Plasmodium falciparum. Woerpel and Clardy developed and tested also many new 1,2-dioxolanes from the derivatives presented in scheme 69.246 For instance, compound $\mathbf{4 4 4}$ is a chimera between a dioxolane and chloroquine, and which shows potent anti-malarial activity on different strains of the parasite; the beneficial effect of the endoperoxide being significant compared to chloroquine alone. Woerpel also found that compound $\mathbf{4 2 7}$, called $\mathrm{FINO}_{2}$ is a very promising anticancer agent, acting in the ferroptosis through GPX4 activation (Figure 9). ${ }^{247}, 248$

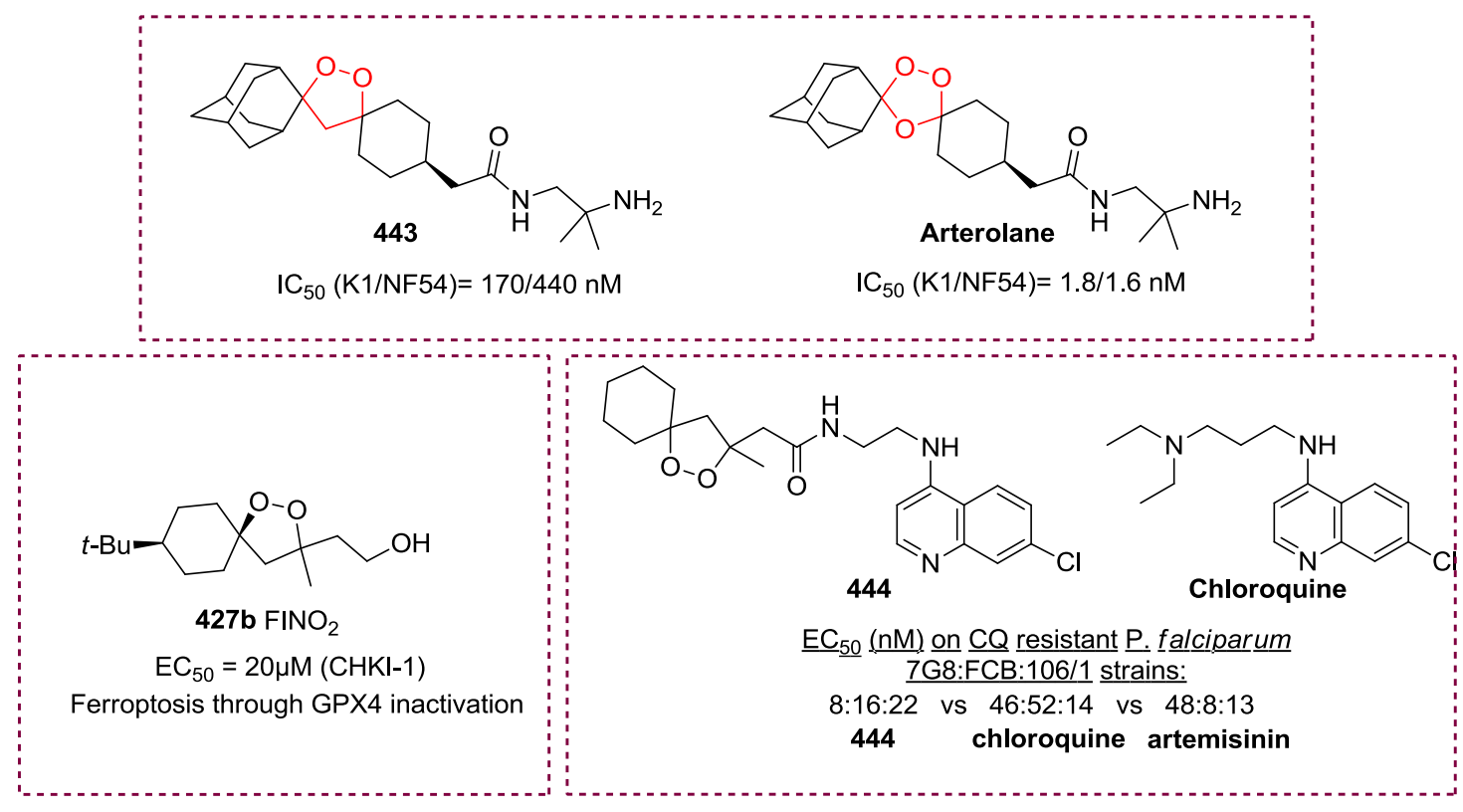

Figure 9. Different bioactive 1,2-dioxolanes prepared by a [3+2] formal cycloaddition.

\subsubsection{Functionalization of Endoperoxyketals and acetals}

The creation of peroxycarbenium species is not only useful for the preparation of 1,2-dioxolanes through a formal [3+2]cycloaddition, but can be also reactive intermediates for the functionalization of endoperoxyketals. Indeed, because the peroxide is already substituted, no [3+2] formal cycloaddition can occur on such species, just a substitution being observed. 
Dussault and coworkers were the first ones to investigate such a transformation. The method was particularly attractive since it would give easy access to many natural products belonging to the family of plakinic acids. ${ }^{249}$ 1,2-Dioxolan-3ol derivatives by themselves are not ideal substrates because of the weak leaving group ability of the hydroxyl group under many Lewis acid conditions. Therefore, a Brønsted acid catalyzed transketalization brought to the synthesis of more potential substrates such as 445a-c giving after Sakurai or Mukaiyama aldol reaction products 449-455, dioxolane 446c affording 455, 447c yielding 457, and finally dioxene 448a leading to compound 458. ${ }^{237}$ The nature of the nucleofuge contributes to the effectiveness of the reaction, thus, 445c exhibits the most effective leaving group ability in these series. Indeed, yields are in general superior with this substituent, due to the ability of methoxyethyl ether to form a chelate with Ti(IV) or Sn(IV). The reaction was working with allylsilanes (Sakurai reaction) but also with silyl enol ether or $S$-silyl ketene acetals (Mukaiyama aldol reaction). The stereoselectivity of the reaction is still difficult to control, thus attempts to produce enantiopure aldol products 454 or 455 with some chiral auxiliary failed. However the Oppolzer auxiliary allowed the separation of diastereomers. ${ }^{250}$ The method was also applied to the synthesis of plakinic acid A (vide supra Figure 2) via the obtention of thioester 457. ${ }^{19}$ The preparation of all possible enantiomers and diastereomers of plakinic acid A in combination with the optical rotation study toward the sign and its absolute value allowed the proposition of an absolute configuration of plakinic acid $\mathrm{A}$. Dussault also were interested in applying a Sakurai reaction on dioxene 448a. The reaction was working for the first time on an endoperoxyacetal derivative to give $\mathbf{4 5 8}$, but the reaction was probably working due to an improved stabilization of the peroxycarbenium species with the unsaturation. ${ }^{237}$ Indeed, the reaction was reported to be ineffective with a saturated derivative (Scheme 72). 


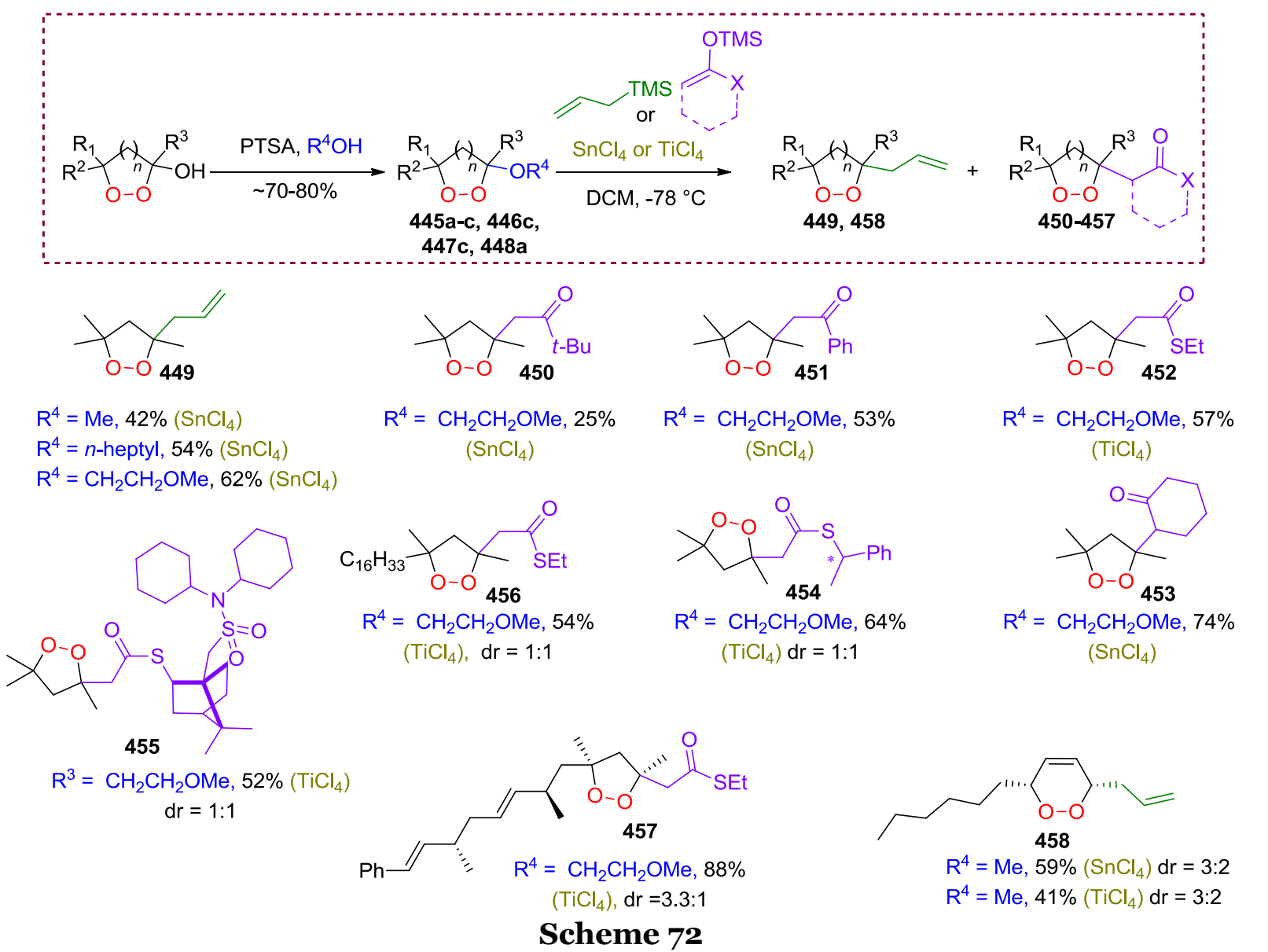

The peroxyendoketals can also be reduced by silanes in acidic conditions. List and cowokers presented some applications of the 1,2-dioxolan-3-ols obtained by an enantioselective conjugate addition of hydrogen peroxide to enones (see paragraph 3.3.2.4, Scheme 52). Thus, by using first a transketalization with $\mathrm{MeOH}$, followed by a treatment with $\mathrm{Et}_{3} \mathrm{SiH}$ and $\mathrm{TiCl}_{4}, 3,5$-disubstituted 1,2-dioxolane 460 could be obtained from 459 over 2 steps. 460 could also be obtained directly by using triflic acid from hydroxyl derivative 459, albeit in lower yield compared to the two-step sequence (Scheme 73).205

We also reported this reduction in a chemical reaction towards the synthesis of a saturated analogue of mycangimycin using List's conditions with triflic acid. This work was presented more precisely in paragraph 3.2.1.3, Scheme 10 (Scheme 73).109 


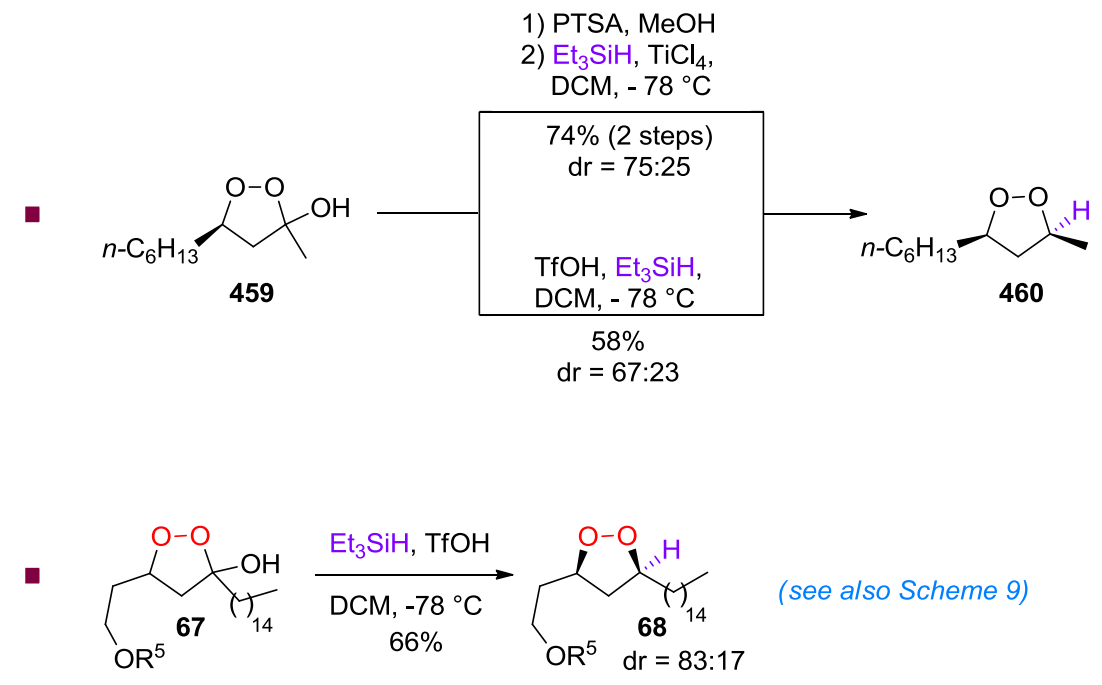

Scheme 73

The two previous examples in Scheme 73 showed how preparing 3,5disubstituted 1,2-dioxolanes by reduction of the peroxyketal, leading to major cisdiastereomers. However the direct preparation of 3,5-disubstituted 1,2-dioxolanes or 1,2-dioxanes from endoperoxyacetals is still unsolved since most of the previous examples were reported from peroxyketals, excluding substrate $\mathbf{4 4 8 a}$, which drove to the formation of $\mathbf{4 5 8}$, because of the enhanced stabilization of peroxycarbenium ion with the unsaturation (see Scheme 72). Our own experience on 3-methoxy-1,2dioxolanes led us to the same conclusion about their poor reactivity. To overcome these difficulties, acetate was found to be a more appropriate nucleofuge. ${ }^{241}$ However, the protocol of acetylation was crucial, since classical conditions $\left(\mathrm{Ac}_{2} \mathrm{O}\right.$, pyridine or DMAP) were unsuccessful due to the reactivity of peroxides towards amines. Lewis acid catalyzed acetylation was in contrast very effective and could provide these corresponding reactive substrates. Addition of different nucleophiles was performed using $\mathrm{SnCl}_{4}$ or $\mathrm{TiCl}_{4}$ as Lewis acids (o.9 equiv). The reaction was allowed with very diverse nucleophiles, from allylsilanes (461-469) to silyl enol ethers of ketone derivatives (472-473), to silanes $(\mathbf{4 7 0}, \mathbf{4 7 1}, \mathbf{4 7 4})$. The results were different by using $\mathrm{TiCl}_{4}$ or $\mathrm{SnCl}_{4}$, the first one giving high trans selectivities but low yields, whereas the second one delivered 1,2-dioxolanes in significant higher yields but with lower diatereoselectivities. It was found that 3,5-disubstituted 1,2-dioxolanes underwent a more pronounced but selective degradation of the cis-diastereomer with $\mathrm{TiCl}_{4}$, rationalizing these results. ${ }^{241}$ (Scheme 74) 


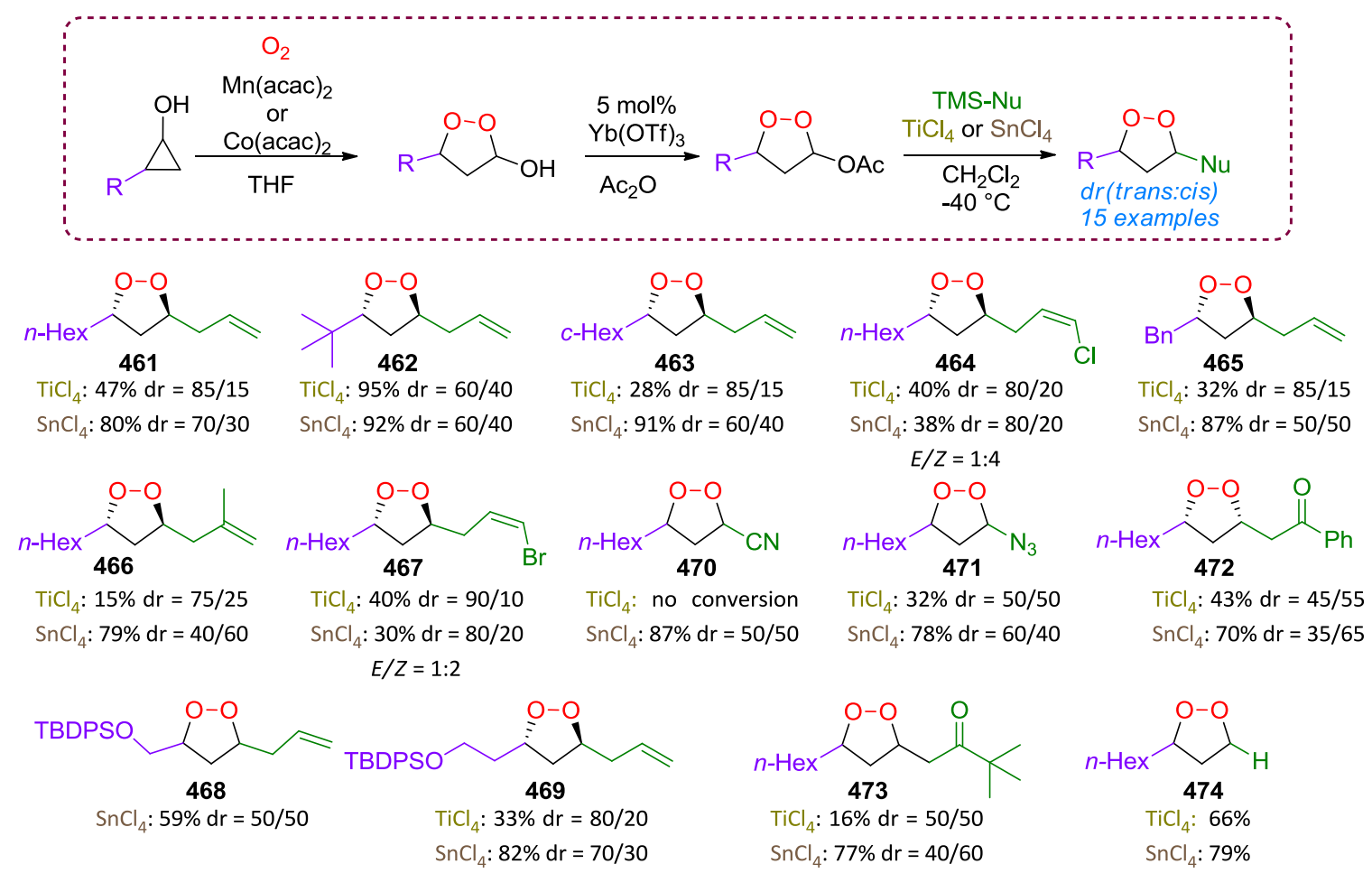

\section{Scheme 74}

Slightly later, we reported the same transformation using improved experimental conditions. Utilization of mild Lewis acids such as $\mathrm{Sc}(\mathrm{OTf})_{3}$ or $\mathrm{InCl}_{3} / \mathrm{TMSCl}$ proved to be very convenient catalysts for such a reaction. ${ }^{21}$ Besides the catalytic amounts of Lewis acids used ( $5 \mathrm{~mol} \%$ ), the room temperature conditions instead of $-40{ }^{\circ} \mathrm{C}$ facilitate the experimental protocol. No significant degradation was observed with these catalysts compared to the previous study. ${ }^{241}$ Moreover, it was found that these conditions expanded the scope of the reaction to other nucleophiles such as $O$ - or $S$-silyl ketene acetals (476 and 477-478, Scheme 75). However, deactivated allylsilanes such as bromo- or chloro-allylsilanes were ineffective with these catalysts $(464 \& 467$, Scheme 75$)$. This concludes, finally, that the two protocols, i.e. stoichiometric amount of a strong Lewis acid or catalytic amount of a mild Lewis acid, are complementary to each other (Scheme 75). 


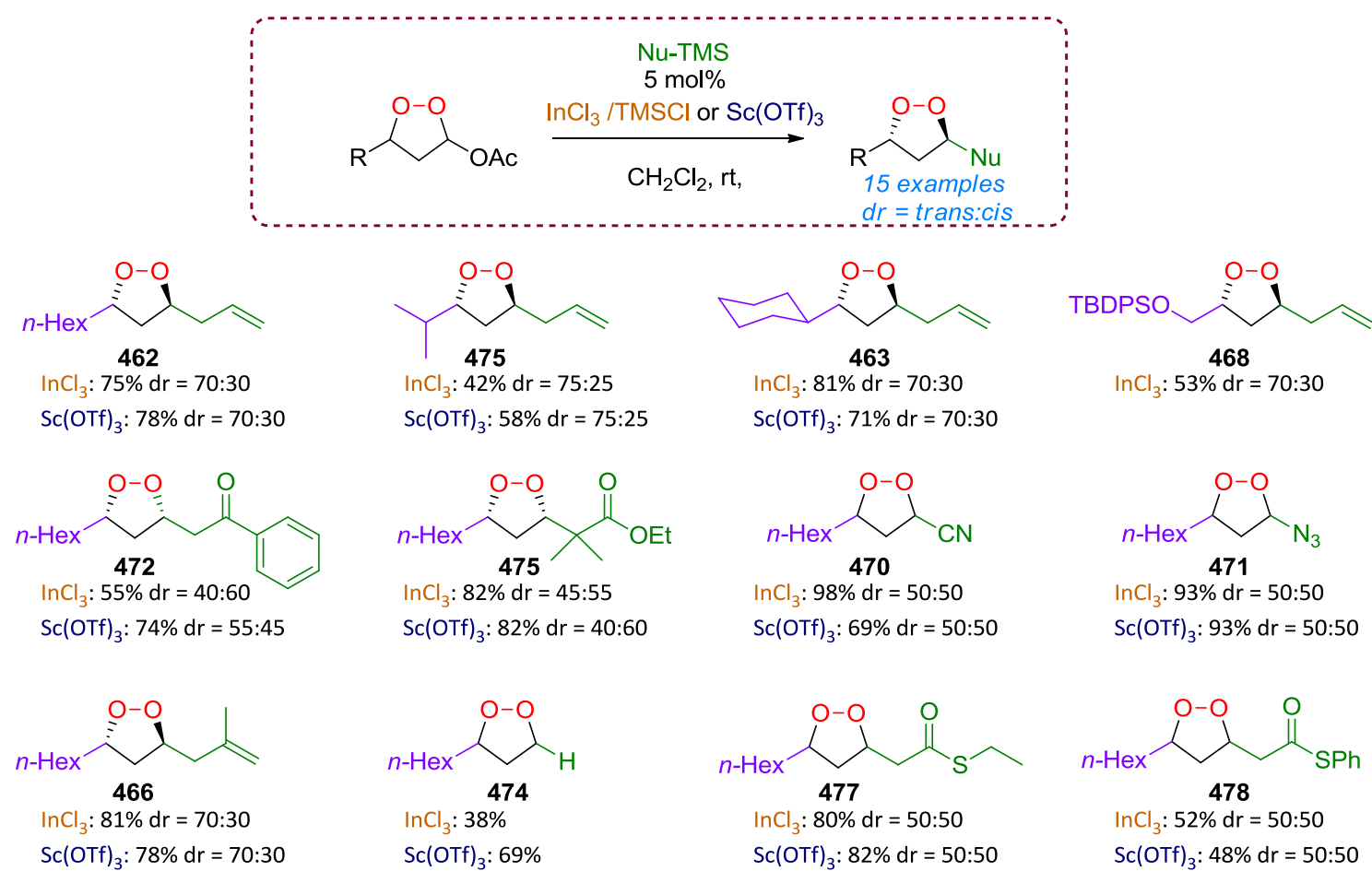

\section{Scheme 75}

This last method was also applied to the synthesis of functionalized 1,2dioxanes. A general approach for the synthesis of 1,2-dioxan-3-ols was developed by us using an oxidative ring expansion of cyclobutanols (see paragraph 3.2.1.3, scheme 11), therefore the access to substituted 1,2-dioxanes could be possible. ${ }^{110}$ Indeed, the reaction between various silylated nucleophiles and 1,2-dioxan-3-yl acetates in presence of $\mathrm{Sc}(\mathrm{OTf})_{3}$ afforded mostly the desired products in good yields. The selectivity, compared to the reaction with 1,2-dioxolan-3-yl acetates, ${ }^{241,251}$ was in general higher, giving the 3,6-cis-isomer as major product. Thus, allylated product 479 was isolated as a sole diastereomer whereas other nucleophiles gave $\mathbf{4 8 0}$ and 481 with more moderated selectivities. 6,6-Disubstituted 1,2-dioxanyl-3-acetates are also viable substrates, and the selectivity towards the cis-diastereomer depends of the nature of the substituents: tert-butyl group discriminating strongly the addition over methyl group to one side $(\mathbf{4 8 2}, \mathbf{4 8 6})$; the selectivity is more moderate with other substituents $(\mathbf{4 8 9}, \mathbf{4 9 0})$. Aromatic substituent gave poor yield for the substitution (491), because it facilitates side reactions such as eliminations (Kornblum-DeLaMare rearrangement). Different nucleophiles could be used from ketones (483) or substituted allylsilanes (484) or siloxyfurans (485). (Scheme 76) 


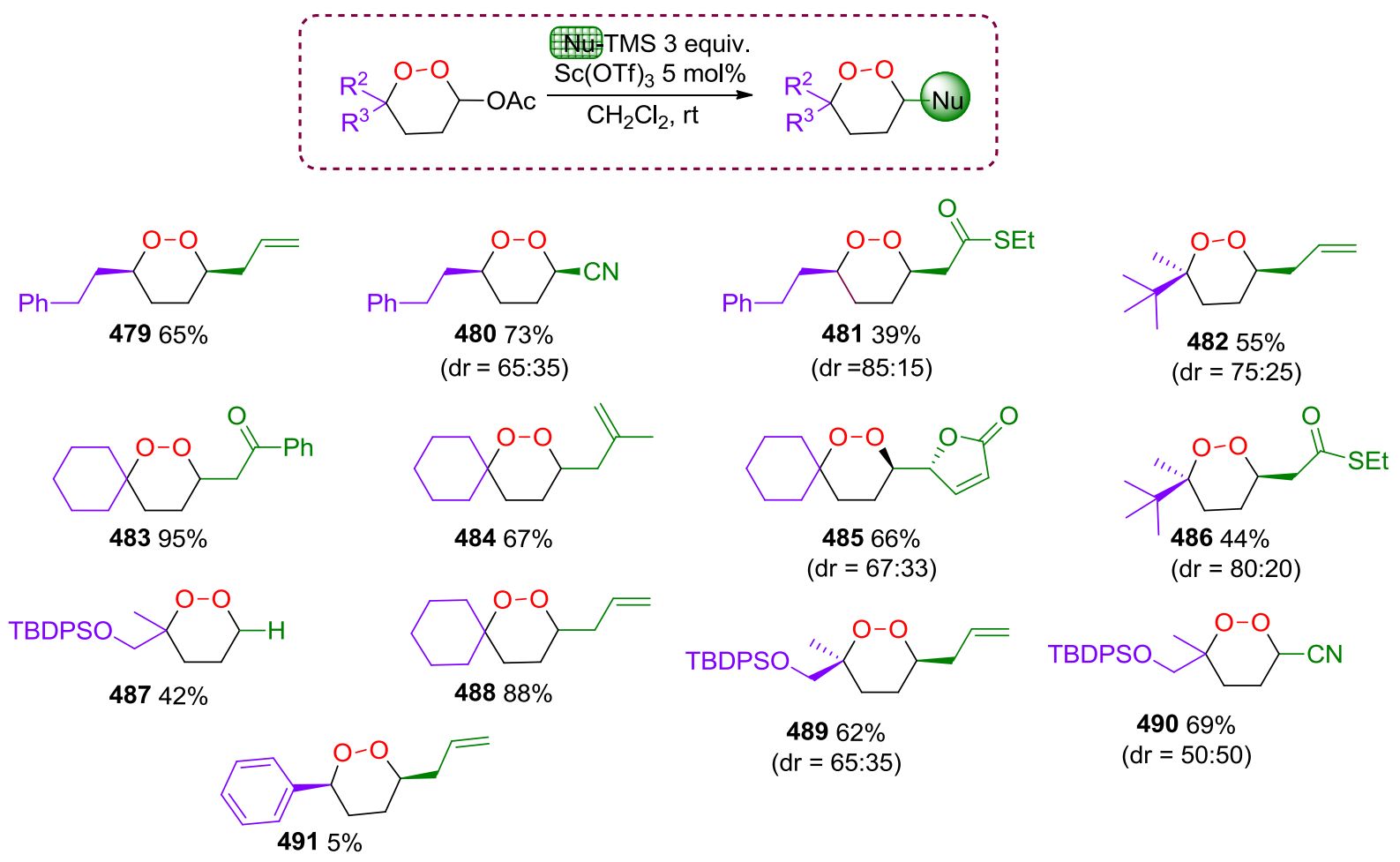

\section{Scheme 76}

\section{Conclusion}

This book chapter reviews the most advanced and popular methods to access to 1,2-dioxolanes and 1,2-dioxanes. The most studied methods are probably the one using radical processes involving oxygen triplet. Indeed, they allow to conveniently and directly obtain the endoperoxides, through a radical chain reaction with a free reagent; however they lack to give the desired product in a diastereoselective or enantioselective manner. Nucleophilic addition of hydroperoxides needs, in contrast, to have generally the hydroperoxy function already present in the molecule to lead to endoperoxides, which consists in a final cyclization; however these process are more prone to give stereoselective and stereospecific reactions. Oxygen singlet is also a popular reagent, especially to performed [4+2] cycloadditions, but is limited to few substrates (ene-reactions) or to the construction of some 1,2-dioxenes or 1,2-dioxanes by extension. The use of peroxycarbenium ion is a more recent method developed from the 90's, which is an interesting tool to access to new structure, in particular 1,2dioxolanes through a formal [3+2] cycloaddition; however an effort will have to be furnished in the future to develop asymmetric versions of this reaction. Many natural products exhibiting a 1,2-dioxane or a 1,2-dioxolane have been reported with 
antibacterial, antifungal, antimalarial, antiviral or cytotoxic activities, a high proportion of which was isolated from marine sponges. However, few total syntheses of these compounds were studied compared to the large number of natural substances reported. This means that still some efforts are needed to access these structures. Efforts to find and develop new methods in a stereoselective fashion have to be conducted in the future to overcome present limitations.

\section{References}

1. Schulz, M.; Kirschke, K. Cyclic Peroxides. In Advances in Heterocyclic Chemistry; Elsevier, 1967; Vol. 8, pp 165-217. https://doi.org/10.1016/S0065-2725(08)60607-8.

2. Terent'ev, A. O.; Borisov, D. A.; Vil', V. A.; Dembitsky, V. M. Beilstein J. Org. Chem. 2014, 10 (1), 34-114. https://doi.org/10.3762/bjoc.10.6.

3. McCullough, K. J. and Nojima, M. Curr. Org. Chem. 2001, 5, 601-636. https://doi.org/10.2174/1385272013375346

4. Pinet, A.; Nguyen, L. T.; Figadère, B.; Ferrié, L. Eur. J. Org. Chem., early view. https://doi.org/10.1002/ejoc.202000980.

5. Korshin, E. E.; Bachi, M. D. Synthesis of Cyclic Peroxides. In PATAl'S Chemistry of Functional Groups; American Cancer Society, 2009. https://doi.org/10.1002/9780470682531.pat0351.

6. Category 5, Compounds with One Saturated Carbon Heteroatom Bond: Peroxides, 1st ed.; Berkessel, Ed.; Georg Thieme Verlag: Stuttgart, 2009. https://doi.org/10.1055/b-003-121800.

7. Liu, D.-Z.; Liu, J.-K. Nat. Prod. Bioprospect. 2013, 3 (5), 161-206. https://doi.org/10.1007/s13659013-0042-7.

8. Casteel, D. A. Nat. Prod. Rep. 1999, 16 (1), 55-73. https://doi.org/10.1039/A705725C.

9. Casteel, D. A. Nat. Prod. Rep. 1992, 9 (4), 289-312. https://doi.org/10.1039/NP9920900289.

10. Phillipson, D. W.; Rinehart, K. L. J. Am. Chem. Soc. 1983, 105 (26), 7735-7736. https://doi.org/10.1021/ja00364a045.

11. Davidson, B. S. J. Org. Chem. 1991, 56 (23), 6722-6724. https://doi.org/10.1021/j000023a048.

12. Horton, P. A.; Longley, R. E.; Kelly-Borges, M.; McConnell, O. J.; Ballas, L. M. J. Nat. Prod. 1994, 57 (10), 1374-1381. https://doi.org/10.1021/np50112a006.

13. Chen, Y.; Killday, K. B.; McCarthy, P. J.; Schimoler, R.; Chilson, K.; Selitrennikoff, C.; Pomponi, S. A.; Wright, A. E. J. Nat. Prod. 2001, 64 (2), 262-264. https://doi.org/10.1021/np000368+.

14. Jiménez-Romero, C.; Ortiz, I.; Vicente, J.; Vera, B.; Rodríguez, A. D.; Nam, S.; Jove, R. J. Nat. Prod. 2010, 73 (10), 1694-1700. https://doi.org/10.1021/np100461t.

15. Sandler, J. S.; Colin, P. L.; Hooper, J. N. A.; Faulkner, D. J. J. Nat. Prod. 2002, 65 (9), 1258-1261. https://doi.org/10.1021/np020228v.

16. Dalisay, D. S.; Quach, T.; Nicholas, G. N.; Molinski, T. F. Angew. Chem. Int. Ed. 2009, 48 (24), 4367-4371. https://doi.org/10.1002/anie.200900888.

17. Dalisay, D. S.; Quach, T.; Molinski, T. F. Org. Lett. 2010, 12 (7), 1524-1527. https://doi.org/10.1021/ol100249v.

18. Jamison, M. T.; Dalisay, D. S.; Molinski, T. F. J. Nat. Prod. 2016, 79 (3), 555-563. https://doi.org/10.1021/acs.jnatprod.5b00951.

19. Dai, P.; Trullinger, T. K.; Liu, X.; Dussault, P. H J. Org. Chem. 2006, 71 (6), 2283-2292. https://doi.org/10.1021/j00522254. 
20. Cafieri, F.; Fattorusso, E.; Taglialatela-Scafati, O.; Ianaro, A. Tetrahedron 1999, 55 (22), 7045 7056. https://doi.org/10.1016/S0040-4020(99)00332-4.

21. Ghosh, I.; Zeng, H.; Kishi, Y. Org. Lett. 2004, 6 (25), 4715-4718. https://doi.org/10.1021/ol048061f.

22. Tian, X.-Y.; Han, J.-W.; Zhao, Q.; Wong, H. N. C. Org. Biomol. Chem. 2014, 12 (22), 3686-3700. https://doi.org/10.1039/C4OB00448E.

23. Jiménez-Romero, C.; Rodríguez, A. D.; Nam, S. Org. Lett. 2017, 19 (6), 1486-1489. https://doi.org/10.1021/acs.orglett.7b00547.

24. Mohammed, R.; Peng, J.; Kelly, M.; Yousaf, M.; Winn, E.; Odde, S.; Bie, Z.; Xie, A.; Doerksen, R. J.; Hamann, M. T. Aust. J. Chem. 2010, 63 (6), 877-885. https://doi.org/10.1071/CH09665.

25. Rudi, A.; Afanii, R.; Gravalos, L. G.; Aknin, M.; Gaydou, E.; Vacelet, J.; Kashman, Y. J. Nat. Prod. 2003, 66 (5), 682-685. https://doi.org/10.1021/np020589a.

26. Barnych, B.; Fenet, B.; Vatèle, J.-M. Tetrahedron 2013, 69 (1), 334-340. https://doi.org/10.1016/j.tet.2012.10.022.

27. Yong, K. W. L.; Barnych, B.; De Voss, J. J.; Vatèle, J.-M.; Garson, M. J. J. Nat. Prod. 2012, 75 (10), 1792-1797. https://doi.org/10.1021/np3005634.

28. Yong, K. W. L.; Lambert, L. K.; Hayes, P. Y.; De Voss, J. J.; Garson, M. J. J. Nat. Prod. 2012, 75 (3), 351-360. https://doi.org/10.1021/np200619q.

29. Yong, K. W. L.; De Voss, J. J.; Hooper, J. N. A.; Garson, M. J. J. Nat. Prod. 2011, 74 (2), 194207. https://doi.org/10.1021/np100620x.

30. Perry, T. L.; Dickerson, A.; Khan, A. A.; Kondru, R. K.; Beratan, D. N.; Wipf, P.; Kelly, M.; Hamann, M. T. Tetrahedron 2001, 57 (8), 1483-1487. https://doi.org/10.1016/S0040-4020(00)01134-0.

31. Fontana, A.; González, M. C.; Gavagnin, M.; Templado, J.; Cimino, G Tetrahedron Lett. 2000, 41 (3), 429-432. https://doi.org/10.1016/S0040-4039(99)02077-8.

32. Durán, R.; Zubía, E.; Ortega, M. J.; Naranjo, S.; Salvá, J. Tetrahedron 2000, 56 (33), 6031-6037. https://doi.org/10.1016/S0040-4020(00)00554-8.

33. Reyes, F.; Rodríguez-Acebes, R.; Fernández, R.; Bueno, S.; Francesch, A.; Cuevas, C. J. Nat. Prod. 2010, 73 (1), 83-85. https://doi.org/10.1021/np900700h.

34. Ovenden, S. P. B.; Capon, R. J. J. Nat. Prod. 1999, 62 (2), 214-218. https://doi.org/10.1021/np980223r.

35. Kashman, Y.; Rotem, M. Tetrahedron Lett. 1979, 20 (19), 1707-1708. https://doi.org/10.1016/S0040-4039(01)93630-5.

36. Sperry, S.; Valeriote, F. A.; Corbett, T. H.; Crews, P. J. Nat. Prod. 1998, 61 (2), 241-247. https://doi.org/10.1021/np970467w.

37. Capon, R. J.; MacLeod, J. K.; Willis, A. C. J. Org. Chem. 1987, 52 (3), 339-342. https://doi.org/10.1021/j000379a004.

38. Tanaka, J.; Higa, T.; Suwanborirux, K.; Kokpol, U.; Bernardinelli, G.; Jefford, C. W. J. Org. Chem. 1993, 58 (11), 2999-3002. https://doi.org/10.1021/j000063a016.

39. Hirade, H.; de Voogd, N. J.; Suzuka, T.; Tanaka, J. Tetrahedron 2019, 75 (33), 4620-4625. https://doi.org/10.1016/..tet.2019.07.005.

40. Albericci, M.; Braekman, J. C.; Daloze, D.; Tursch, B. Tetrahedron 1982, 38 (13), 1881-1890. https://doi.org/10.1016/0040-4020(82)80037-9.

41. Wells, R. J. Tetrahedron Lett. 1976, 17 (30), 2637-2638. https://doi.org/10.1016/S00404039(00)91755-6.

42. Quinoa, E.; Kho, E.; Manes, L. V.; Crews, P.; Bakus, G. J. J. Org. Chem. 1986, 51 (22), 4260 4264. https://doi.org/10.1021/j000372a029.

43. Dussault, P. H.; Woller, K. R. J. Am. Chem. Soc. 1997, 119 (16), 3824-3825. https://doi.org/10.1021/ja970174p. 
44. Yosief, T.; Rudi, A.; Wolde-ab, Y.; Kashman, Y. J. Nat. Prod. 1998, 61 (4), 491-493. https://doi.org/10.1021/np970407q.

45. Fontana, A.; d'Ippolito, G.; D'Souza, L.; Mollo, E.; J. Nat. Prod. 2001, 64 (1), 131-133. https://doi.org/10.1021/np0002435.

46. Chianese, G.; Fattorusso, E.; Scala, F.; Teta, R.; Calcinai, B.; Bavestrello, G.; Dien, H. A.; Kaiser, M.; Tasdemir, D.; Taglialatela-Scafati, O. Org. Biomol. Chem. 2012, 10 (35), 7197-7207. https://doi.org/10.1039/C2OB26124C.

47. Kobayashi, M.; Kondo, K.; Kitagawa, I. Chem. Pharm. Bull. 1993, 41 (7), 1324-1326. https://doi.org/10.1248/cpb.41.1324.

48. Chen, S.-P.; Ahmed, A. F.; Dai, C.-F.; Lu, C.-K.; Hu, W.-P.; Wang, J.-J.; Sheu, J.-H. Tetrahedron 2006, 62 (29), 6802-6807. https://doi.org/10.1016/j.tet.2006.04.088.

49. Ahmed, A. F.; Kuo, Y.-H.; Dai, C.-F.; Sheu, J.-H. J. Nat. Prod. 2005, 68 (8), 1208-1212. https://doi.org/10.1021/np050114u.

50. Chao, C.-H.; Hsieh, C.-H.; Chen, S.-P.; Lu, C.-K.; Dai, C.-F.; Wu, Y.-C.; Sheu, J.-H. Tetrahedron Lett. 2006, 47 (13), 2175-2178. https://doi.org/10.1016/j.tetlet.2006.01.107.

51. Uchio, Y.; Eguchi, S.; Kuramoto, J.; Nakayama, M.; Hase, T. Tetrahedron Lett. 1985, 26 (37), 4487-4490. https://doi.org/10.1016/S0040-4039(00)88937-6.

52. Sawant, S. S.; Youssef, D. T. A.; Sylvester, P. W.; Wali, V.; Sayed, K. A. E. Nat. Prod. Commun. 2019. https://doi.org/10.1177/1934578X0700200201.

53. Umeyama, A.; Machida, M.; Nozaki, M.; Arihara, S. J. Nat. Prod. 1998, 61 (11), 1435-1436. https://doi.org/10.1021/np980210d.

54. Huang, X.-C.; Li, J.; Li, Z.-Y.; Shi, L.; Guo, Y.-W. J. Nat. Prod. 2008, 71 (8), 1399-1403. https://doi.org/10.1021/np8002035.

55. Ueoka, R.; Nakao, Y.; Kawatsu, S.; Yaegashi, J.; Matsumoto, Y.; Matsunaga, S.; Furihata, K.; van Soest, R. W. M.; Fusetani, N. J. Org. Chem. 2009, 74 (11), 4203-4207. https://doi.org/10.1021/j0900380f.

56. Festa, C.; De Marino, S.; D’Auria, M. V.; Deharo, E.; Gonzalez, G.; Deyssard, C.; Petek, S.; Bifulco, G.; Zampella, A. Tetrahedron 2012, 68 (49), 10157-10163. https://doi.org/10.1016/i.tet.2012.09.106.

57. Wallach, O. Liebigs Ann. 1912, 392 (1), 49-75. https://doi.org/10.1002/ilac.19123920104.

58. Labbe, C.; Castillo, M.; Connolly, J. D. Phytochemistry 1993, 34 (2), 441-444. https://doi.org/10.1016/0031-9422(93)80026-O.

59. Liang, X. T.; Yu, D. Q.; Wu, W. L.; Rang, H. C., Acta Chim. Sinica, 1979, 37, 215. http://siocjournal.cn/Jwk hxxb/EN/Y1979/V37/I3/215

60. Zhang, L.; Zhou, W.-S.; Xu, X.-X. J. Chem. Soc., Chem. Commun. 1988, 8, 523-524. https://doi.org/10.1039/C39880000523.

61. Xu, X.-X.; Dong, H.-Q. Tetrahedron Lett. 1994, 35 (50), 9429-9432. https://doi.org/10.1016/S0040-4039(00)78561-3.

62. Rücker, G.; Breitmaier, E.; Mayer, R.; Manns, D. Arch. Pharm. 1987, 320 (5), 437-441. https://doi.org/10.1002/ardp.19873200510.

63. Rücker, G.; Manns, D.; Wilbert, S. Arch. Pharm. 1993, 326 (8), 457-460. https://doi.org/10.1002/ardp.19933260806.

64. Duong, T.-H.; Beniddir, M. A.; Genta-Jouve, G.; Nguyen, H.-H.; Nguyen, D.-P.; Nguyen, T.-A.-T.; Mac, D.-H.; Boustie, J.; Nguyen, K.-P.-P.; Chavasiri, W.; Le Pogam, P. Fitoterapia 2019, 135, 44 51. https://doi.org/10.1016/j.fitote.2019.04.001.

65. Ma, W.-H.; Tan, C.-M.; He, J.-C.; Duan, P.-S.; Qin, L.-P. Chem. Nat. Compd. 2011, 47 (5), 713715. https://doi.org/10.1007/s10600-011-0042-y.

66. Rustaiyan, A.; Sigari, H.; Jakupovic, J.; Grenz, M. Phytochemistry 1989, 28 (10), 2723-2725. https://doi.org/10.1016/S0031-9422(00)98075-0. 
67. Hashidoko, Y.; Tahara, S.; Mizutani, J. Phytochemistry 1989, 28 (2), 425-430. https://doi.org/10.1016/0031-9422(89)80026-3.

68. Itokawa, H.; Morita, H.; Osawa, K.; Watanabe, K.; litaka, Y. Chem. Pharm. Bull. 1987, 35 (7), 2849-2859. https://doi.org/10.1248/cpb.35.2849.

69. Todorova, M.; Vogler, B.; Tsankova, E. 2000, 55 (9-10), 840-842. https://doi.org/10.1515/znc2000-9-1028.

70. O’Mathúna, D. P.; Doskotch, R. W. J. Nat. Prod. 1995, 58 (9), 1407-1418. https://doi.org/10.1021/np50123a011.

71. Adelekan, A. M.; Prozesky, E. A.; Hussein, A. A.; Ureña, L. D.; van Rooyen, P. H.; Liles, D. C.; Meyer, J. J. M.; Rodríguez, B. J. Nat. Prod. 2008, 71 (11), 1919-1922. https://doi.org/10.1021/np800333r.

72. Xuan, J.; Zhu, A.; Ma, B.; Ding, H. Org. Lett. 2018, 20 (13), 4153-4156. https://doi.org/10.1021/acs.orglett.8b01875.

73. Loyola, L. A.; Morales, G.; Rodriguez, B.; Jiménez-Barbero, J.; Torre, M. C. de la; Perales, A.; Torres, M. R. Tetrahedron 1990, 46 (15), 5413-5420. https://doi.org/10.1016/S00404020(01)87848-0.

74. Tan, J.-M.; Qiu, Y.-H.; Tan, X.-Q.; Tan, C.-H. Helv. Chim. Acta 2011, 94 (9), 1697-1702. https://doi.org/10.1002/hlca.201100166.

75. Chiang, Y.-M.; Kuo, Y.-H. J. Nat. Prod. 2001, 64 (4), 436-439. https://doi.org/10.1021/np0004808.

76. Dong, J.-W.; Cai, L.; Li, X.-J.; Peng, L.; Xing, Y.; Mei, R.-F.; Wang, J.-P.; Ding, Z.-T. Fitoterapia 2016, 109, 212-216. https://doi.org/10.1016/j.fitote.2015.12.019.

77. Wang, L.; He, H.-P.; Di, Y.-T.; Zhang, Y.; Hao, X.-J. Tetrahedron Lett. 2012, 53 (13), 1576-1578. https://doi.org/10.1016/j.tetlet.2012.01.060.

78. Lee, S.-S.; Lin, Y.-S.; Chen, C.-K. J. Nat. Prod. 2009, 72 (7), 1249-1252. https://doi.org/10.1021/np9000653.

79. Wieland, P.; Prelog, V. Helv. Chim. Acta 1947, 30 (4), 1028-1030. https://doi.org/10.1002/hlca.19470300414.

80. Li, H.; Huang, H.; Shao, C.; Huang, H.; Jiang, J.; Zhu, X.; Liu, Y.; Liu, L.; Lu, Y.; Li, M.; Lin, Y.; She, Z. J. Nat. Prod. 2011, 74 (5), 1230-1235. https://doi.org/10.1021/np200164k.

81. Chokpaiboon, S.; Sommit, D.; Teerawatananond, T.; Muangsin, N.; Bunyapaiboonsri, T.; Pudhom, K. J. Nat. Prod. 2010, 73 (5), 1005-1007. https://doi.org/10.1021/np100103j.

82. Liu, L.; Niu, S.; Lu, X.; Chen, X.; Zhang, H.; Guo, L.; Che, Y. Chem. Commun. 2010, 46 (3), 460 462. https://doi.org/10.1039/B918330B.

83. Scott, J. J.; Oh, D.-C.; Yuceer, M. C.; Klepzig, K. D.; Clardy, J.; Currie, C. R. Science 2008, 322 (5898), 63-63. https://doi.org/10.1126/science.1160423.

84. Oh, D.-C.; Scott, J. J.; Currie, C. R.; Clardy, J Org. Lett. 2009, 11 (3), 633-636. https://doi.org/10.1021/ol802709x.

85. Hamberg, M.; Samuelsson, B. Proc. Natl. Acad. Sci. USA 1973, 70 (3), 899-903. https://doi.org/10.1073/pnas.70.3.899.

86. Moncada, S.; Vane, J. R. Pharmacol. Rev. 1978, 30 (3), 293-331. http://pharmrev.aspetjournals.org/content/30/3/293.

87. Nissen, J. H.; Drews, T.; Schröder, B.; Beckers, H.; Steinhauer, S.; Riedel, S. Chem. Eur. J. 2019, 25 (64), 14721-14727. https://doi.org/10.1002/chem.201903620.

88. Daniel Little, R.; Losiski-Dang, L.; Venegas, M. G.; Merlic, C. Tetrahedron Lett. 1983, 24 (42), 4499-4502. https://doi.org/10.1016/S0040-4039(00)85938-9.

89. Wilson, R. M.; Schnapp, K. A.; Merwin, R. K.; Ranganthan, R.; Moats, D. L.; Conrad, T. T. J. Org. Chem. 1986, 51 (21), 4028-4035. https://doi.org/10.1021/j000371a021. 
90. Chou, C.-H.; Trahanovsky, W. S. J. Org. Chem. 1995, 60 (17), 5449-5451. https://doi.org/10.1021/j000122a024.

91. (1) Mizuno, K.; Kamiyama, N.; Ichinose, N.; Otsuji, Y. Tetrahedron 1985, 41 (11), 2207-2214. https://doi.org/10.1016/S0040-4020(01)96594-9.

92. (1) Maeda, H.; Nakagawa, H.; Mizuno, K. Photochem. Photobiol. Sci. 2003, 2 (11), 1056-1058. https://doi.org/10.1039/B306186H.

93. (1) Lu, Z.; Parrish, J. D.; Yoon, T. P. Tetrahedron 2014, 70 (27-28), 4270-4278. https://doi.org/10.1016/..tet.2014.02.045.

94. (1) Mizuno, K.; Tamai, T.; Hashida, I.; Otsuji, Y.; Kuriyama, Y.; Tokumaru, K. J. Org. Chem. 1994, 59 (24), 7329-7334. https://doi.org/10.1021/j000103a025.

95. (1) Parrish, J. D.; Ischay, M. A.; Lu, Z.; Guo, S.; Peters, N. R.; Yoon, T. P. Org. Lett. 2012, 14 (6), 1640-1643. https://doi.org/10.1021/ol300428q.

96. (1) Gesmundo, N. J.; Nicewicz, D. A. Beilstein J. Org. Chem. 2014, 10 (1), 1272-1281. https://doi.org/10.3762/bjoc.10.128.

97. (1) Qiao, X.; Biswas, S.; Wu, W.; Zhu, F.; Tung, C.-H.; Wang, Y. Tetrahedron 2018, 74 (20), 24212427. https://doi.org/10.1016/j.tet.2018.03.040.

98. (1) Wimalasena, K.; Wickman, H. B.; Mahindaratne, M. P. D. Eur. J. Org. Chem. 2001, 2001 (20), 3811-3817. https://doi.org/10.1002/1099-0690(200110)2001:20<3811::AID-EJOC3811>3.0.CO;26.

99. Ouhamou, N.; Six, Y. Org. Biomol. Chem. 2003, 1 (17), 3007-3009. https://doi.org/10.1039/B306719J.

100. Madelaine, C.; Six, Y.; Buriez, O. Angew. Chem. 2007, 119 (42), 8192-8195. https://doi.org/10.1002/ange.200702903.

101. Madelaine, C.; Buriez, O.; Crousse, B.; Florent, I.; Grellier, P.; Retailleau, P.; Six, Y. Org. Biomol. Chem. 2010, 8 (24), 5591-5601. https://doi.org/10.1039/C0OB00308E.

102. Nuter, F.; Dimé, A. K. D.; Chen, C.; Bounaadja, L.; Mouray, E.; Florent, I.; Six, Y.; Buriez, O.; Marinetti, A.; Voituriez, A. Chem. Eur. J.2015, 21 (14), 5584-5593. https://doi.org/10.1002/chem.201406138.

103. Xiong, C.; Cheng, K.; Wang, J.; Yang, F.; Lu, J.; Zhou, Q. J. Org. Chem. 2020, 85 (14), 93869395. https://doi.org/10.1021/acs.joc.0c00652.

104. Budde, S.; Goerdeler, F.; Floß, J.; Kreitmeier, P.; Hicks, E. F.; Moscovitz, O.; Seeberger, P. H.; Davies, H. M. L.; Reiser, O. Org. Chem. Front. 2020, 7 (14), 1789-1795. https://doi.org/10.1039/D0QO00168F.

105. Gibson, D. H.; DePuy, C. H. Tetrahedron Lett. 1969, 10 (27), 2203-2206. https://doi.org/10.1016/S0040-4039(01)88122-3.

106. Kulinkovich, O. G.; Astashko, D. A.; Tyvorskii, V. I.; Ilyina, N. A. Synthesis 2001, 2001 (10), 14531455. https://doi.org/10.1055/s-2001-16089.

107. Elek, G. Z.; Borovkov, V.; Lopp, M.; Kananovich, D. G. Org. Lett. 2017, 19 (13), 3544-3547. https://doi.org/10.1021/acs.orglett.7b01519.

108. Han, W.-B.; Li, S.-G.; Lu, X.-W.; Wu, Y. Eur. J. Org. Chem. 2014, 2014 (18), 3841-3846. https://doi.org/10.1002/ejoc.201402175.

109. Nguyen, T. L.; Ferrié, L.; Figadère, B. Tetrahedron Lett. 2016, 57 (47), 5286-5289. https://doi.org/10.1016/j.tetlet.2016.10.051.

110. M. Martín López; Nicolas, J.; Pinet, Alexis; Figadère, Bruno; Ferrié, L. ChemRxiv 2020, Preprint. https://doi.org/10.26434/chemrxiv.13386917.v1.

111. Boto, A.; Betancor, C.; Suárez, E. Tetrahedron Lett. 1994, 35 (30), 5509-5512. https://doi.org/10.1016/S0040-4039(00)73537-4.

112. Iwahama, T.; Sakaguchi, S.; Ishii, Y. Chem. Commun. 2000, No. 23, 2317-2318. https://doi.org/10.1039/B007182J. 
113. Kumabe, R.; Nishino, H.; Yasutake, M.; Nguyen, V.-H.; Kurosawa, K. Tetrahedron Lett. 2001, 42 (1), 69-72. https://doi.org/10.1016/S0040-4039(00)01884-0.

114. Asahi, K.; Nishino, H. Tetrahedron 2005, 61 (47), 11107-11124. https://doi.org/10.1016/j.tet.2005.09.029.

115. Asahi, K.; Nishino, H. Tetrahedron 2008, 64 (8), 1620-1634. https://doi.org/10.1016/..tet.2007.12.017.

116. Asahi, K.; Nishino, H. Eur. J. Org. Chem. 2008, 2008 (14), 2404-2416. https://doi.org/10.1002/ejoc.200701232.

117. Linker, T. J. Organomet. Chem. 2002, 661 (1), 159-167. https://doi.org/10.1016/S0022328X(02)01824-7.

118. Kumabe, R.; Nishino, H. Tetrahedron Lett. 2004, 45 (4), 703-706. https://doi.org/10.1016/i.tetlet.2003.11.054.

119. Nishino, H.; Kumabe, R.; Hamada, R.; Yakut, M. Tetrahedron 2014, 70 (7), 1437-1450. https://doi.org/10.1016/..tet.2014.01.013.

120. Biyogo, A. M.; Curti, C.; El-Kashef, H.; Khoumeri, O.; Terme, T.; Vanelle, P. RSC Adv. 2016, 7 (1), 106-111. https://doi.org/10.1039/C6RA25138B.

121. Nishino, H.; Haque, A. Heterocycles, 2011, 83 (8), 1783. https://doi.org/10.3987/COM-11-12241.

122. Daeppen, C.; Kaiser, M.; Neuburger, M.; Gademann, K. Org. Lett. 2015, 17 (21), 5420-5423. https://doi.org/10.1021/acs.orglett.5b02773.

123. Christoffers, J.; Werner, T.; Frey, W.; Baro, A. Eur. J. Org. Chem. 2003, 2003 (24), 4879-4886. https://doi.org/10.1002/ejoc.200300439.

124. Rössle, M.; Werner, T.; Frey, W.; Christoffers, J. Eur. J. Org. Chem. 2005, 2005 (23), 5031-5038. https://doi.org/10.1002/ejoc.200500487.

125. Tsubusaki, T.; Nishino, H. Tetrahedron 2009, 65 (18), 3745-3752. https://doi.org/10.1016/.tet.2009.02.045.

126. IsayamaShigeru; MukaiyamaTeruaki. Chem. Lett. 2006. https://doi.org/10.1246/cl.1989.573.

127. Isayama, S. Bull. Chem. Soc. Jpn. 1990, 63 (5), 1305-1310. https://doi.org/10.1246/bcsi.63.1305.

128. Tokuyasu, T.; Kunikawa, S.; Masuyama, A.; Nojima, M. Org. Lett. 2002, 4 (21), 3595-3598. https://doi.org/10.1021/ol0201299.

129. Tokuyasu, T.; Kunikawa, S.; Abe, M.; Masuyama, A.; Nojima, M.; Kim, H.-S.; Begum, K.; Wataya, Y. J. Org. Chem. 2003, 68 (19), 7361-7367. https://doi.org/10.1021/j0030107f.

130. Tokuyasu, T.; Kunikawa, S.; McCullough, K. J.; Masuyama, A.; Nojima, M. J. Org. Chem. 2005, 70 (1), 251-260. https://doi.org/10.1021/jo048359j.

131. Wu, J.-M.; Kunikawa, S.; Tokuyasu, T.; Masuyama, A.; Nojima, M.; Kim, H.-S.; Wataya, Y. Tetrahedron 2005, 61 (42), 9961-9968. https://doi.org/10.1016/j.tet.2005.08.025.

132. Chen, H.-J.; Wu, Y. Org. Lett. 2015, 17 (3), 592-595. https://doi.org/10.1021/ol503603t.

133. Hu, X.; Musacchio, A. J.; Shen, X.; Tao, Y.; Maimone, T. J. J. Am. Chem. Soc. 2019, 141 (37), 14904-14915. https://doi.org/10.1021/jacs.9b08001.

134. Li, Q.; Zhao, K.; Peuronen, A.; Rissanen, K.; Enders, D.; Tang, Y. J. Am. Chem. Soc. 2018, 140 (5), 1937-1944. https://doi.org/10.1021/jacs.7b12903.

135. Kharasch, M. S.; Nudenberg, W.; Mantell, G. J. J. Org. Chem. 1951, 16 (4), 524-532. https://doi.org/10.1021/j001144a005.

136. Beckwith, A. L. J.; Wagner, R. D. J. Am. Chem. Soc. 1979, 101 (23), 7099-7100. https://doi.org/10.1021/ja00517a069.

137. Barker, P. J.; Beckwith, A. L. J.; Fung, Y. Tetrahedron Lett. 1983, 24 (1), 97-100. https://doi.org/10.1016/S0040-4039(00)81337-4. 
138. Beckwith, A. L. J.; Wagner, R. D. J. Chem. Soc., Chem. Commun. 1980, No. 11, 485-486. https://doi.org/10.1039/C39800000485.

139. Bachi, M. D.; Korshin, E. E. Synlett 1998, 1998 (2), 122-124. https://doi.org/10.1055/s-19981587.

140. Korshin, E. E.; Hoos, R.; Szpilman, A. M.; Konstantinovski, L.; Posner, G. H.; Bachi, M. D. Tetrahedron 2002, 58 (12), 2449-2469. https://doi.org/10.1016/S0040-4020(02)00126-6.

141. Szpilman, A. M.; Korshin, E. E.; Rozenberg, H.; Bachi, M. D. J. Org. Chem. 2005, 70 (9), 36183632. https://doi.org/10.1021/j0050074z.

142. O’Neill, P. M.; Verissimo, E.; Ward, S. A.; Davies, J.; Korshin, E. E.; Araujo, N.; Pugh, M. D.; Cristiano, M. L. S.; Stocks, P. A.; Bachi, M. D. Bioorganic \& Medicinal Chem. Lett. 2006, 16 (11), 2991-2995. https://doi.org/10.1016/j.bmcl.2006.02.059.

143. Bachi, M. D.; Korshin, E. E.; Hoos, R.; Szpilman, A. M.; Ploypradith, P.; Xie, S.; Shapiro, T. A.; Posner, G. H. A. J. Med. Chem. 2003, 46 (12), 2516-2533. https://doi.org/10.1021/im020584a.

144. O’Neill, P. M.; Stocks, P. A.; Pugh, M. D.; Araujo, N. C.; Korshin, E. E.; Bickley, J. F.; Ward, S. A.; Bray, P. G.; Pasini, E.; Davies, J.; Verissimo, E.; Bachi, M. D. Angew. Chem. Int. Ed. 2004, 43 (32), 4193-4197. https://doi.org/10.1002/anie.200453859.

145. Feldman, K. S.; Parvez, M. J. Am. Chem. Soc. 1986, 108 (6), 1328-1330. https://doi.org/10.1021/ja00266a051.

146. Feldman, K. S.; Simpson, R. E. J. Am. Chem. Soc. 1989, 111 (13), 4878-4886. https://doi.org/10.1021/ja00195a047.

147. Feldman, K. S.; Simpson, R. E. Tetrahedron Lett. 1989, 30 (50), 6985-6988. https://doi.org/10.1016/S0040-4039(01)93404-5.

148. Sun, X.-Y.; Tian, X.-Y.; Li, Z.-W.; Peng, X.-S.; Wong, H. N. C. Chem. Eur. J. 2011, 17 (21), 5874 5880. https://doi.org/10.1002/chem.201003309.

149. Brown, H. C.; Midland, M. M. J. Am. Chem. Soc. 1971, 93 (16), 4078-4080. https://doi.org/10.1021/ja00745a060.

150. Klement, I.; Lütjens, H.; Knochel, P. Tetrahedron 1997, 53 (27), 9135-9144. https://doi.org/10.1016/S0040-4020(97)00603-0.

151. Mata, S.; González, J.; Vicente, R.; López, L. A. Eur. J. Org. Chem. 2016, 2016 (15), 2681-2687. https://doi.org/10.1002/ejoc.201600393.

152. Berkessel, A.; Vogl, N. Synthetic Uses of Peroxides. In PATAl'S Chemistry of Functional Groups; American Cancer Society, 2009. https://doi.org/10.1002/9780470682531.pat0352.

153. Gandhi, H.; O'Reilly, K.; Gupta, M. K.; Horgan, C.; O'Leary, E. M.; O'Sullivan, T. P. Advances in the Synthesis of Acyclic Peroxides. RSC Adv. 2017, 7 (32), 19506-19556. https://doi.org/10.1039/C6RA28489B.

154. An, X.; Zha, Q.; Wu, Y. Org. Lett. 2019, 21 (5), 1542-1546. https://doi.org/10.1021/acs.orglett.9b00425.

155. Li, Y.; Hao, H.-D.; Wu, Y. Org. Lett. 2009, 11 (12), 2691-2694. https://doi.org/10.1021/ol900811m.

156. Groendyke, B. J.; Modak, A.; Cook, S. P. J. Org. Chem. 2019, 84 (20), 13073-13091. https://doi.org/10.1021/acs.joc.9b01979.

157. Sakurai, S.; Kato, T.; Sakamoto, R.; Maruoka, K. Tetrahedron 2019, 75 (2), 172-179. https://doi.org/10.1016/j.tet.2018.11.048.

158. Bloodworth, A. J.; Spencer, M. D. J. Organomet. Chem. 1990, 386 (3), 299-304. https://doi.org/10.1016/0022-328X(90)80002-H.

159. Terent'ev, A. O.; Krylov, I. B.; Borisov, D. A.; Nikishin, G. I. Synthesis 2007, 2007 (19), 2979-2986. https://doi.org/10.1055/s-2007-990776.

160. Stephenson, L. M. Tetrahedron Lett. 1980, 21 (11), 1005-1008. https://doi.org/10.1016/S00404039(00)78824-1. 
161. Velluz, L.; Muller, G.; Mathieu, J.; Poittevin, A. Tetrahedron 1960, 9 (3), 145-148. https://doi.org/10.1016/0040-4020(60)80002-6.

162. Carless, H. A. J.; Batten, R. J. Tetrahedron Lett. 1982, 23 (45), 4735-4738. https://doi.org/10.1016/S0040-4039(00)85700-7.

163. Boukouvalas, J.; Pouliot, R.; Fréchette, Y. Tetrahedron Lett. 1995, 36 (24), 4167-4170. https://doi.org/10.1016/0040-4039(95)00714-N.

164. Corey, E. J.; Nicolaou, K. C.; Shibasaki, M.; Machida, Y.; Shiner, C. S. Tetrahedron Lett. 1975, 16 (37), 3183-3186. https://doi.org/10.1016/S0040-4039(00)91450-3.

165. Salomon, M. F.; Salomon, R. G. J. Am. Chem. Soc. 1977, 99 (10), 3500-3501. https://doi.org/10.1021/ja00452a051.

166. Bloodworth, A. J.; Eggelte, H. J. Tetrahedron Lett. 1981, 22 (2), 169-172. https://doi.org/10.1016/0040-4039(81)80179-7.

167. Takahashi, K.; Kishi, M. J. Chem. Soc., Chem. Commun. 1987, 10, 722-724. https://doi.org/10.1039/C39870000722.

168. Takahashi, K.; Kishi, M. Tetrahedron 1988, 44 (15), 4737-4746. https://doi.org/10.1016/S00404020(01)86176-7.

169. Ghorai, P.; Dussault, P. H.; Hu, C. Org. Lett. 2008, 10 (12), 2401-2404. https://doi.org/10.1021/ol800657m.

170. Xu, C.; Schwartz, C.; Raible, J.; Dussault, P. H. Tetrahedron 2009, 65 (47), 9680-9685. https://doi.org/10.1016/i.tet.2009.09.068.

171. Schneider, M.-A.; Seifert, K. Eur. J. Org. Chem. 2017, 2017 (45), 6739-6746. https://doi.org/10.1002/ejoc.201700922.

172. Griesbeck, A. G.; Goldfuss, B.; Jäger, C.; Brüllingen, E.; Lippold, T.; Kleczka, M. ChemPhotoChem 2017, 1 (5), 213-221. https://doi.org/10.1002/cptc.201600041.

173. Wang, Y.; Chen, B.; He, X.; Gui, J. J. Am. Chem. Soc. 2020, 142 (11), 5007-5012. https://doi.org/10.1021/jacs.0c00363.

174. Xu, X.-X.; Dong, H.-Q. J. Org. Chem. 1995, 60 (10), 3039-3044. https://doi.org/10.1021/j000115a019.

175. Gemma, S.; Gabellieri, E.; Sanna Coccone, S.; Martí, F.; Taglialatela-Scafati, O.; Novellino, E.; Campiani, G.; Butini, S. J. Org. Chem. 2010, 75 (7), 2333-2340. https://doi.org/10.1021/jo1001559.

176. Gemma, S.; Martí, F.; Gabellieri, E.; Campiani, G.; Novellino, E.; Butini, S. Tetrahedron Letters 2009, 50 (41), 5719-5722. https://doi.org/10.1016/j.tetlet.2009.07.137.

177. Barnych, B.; Vatèle, J.-M. Tetrahedron 2012, 68 (19), 3717-3724. https://doi.org/10.1016/j.tet.2012.03.024.

178. Hu, L.; Lu, X.; Deng, L. J. Am. Chem. Soc. 2015, 137 (26), 8400-8403. https://doi.org/10.1021/jacs.5b05345.

179. Dai, P.; Dussault, P. H. Org. Lett. 2005, 7 (20), 4333-4335. https://doi.org/10.1021/ol051407h.

180. Nixon, J. R.; Cudd, M. A.; Porter, N. A. J. Org. Chem. 1978, 43 (21), 4048-4052. https://doi.org/10.1021/j000415a014.

181. Bloodworth, A. J.; Loveitt, M. E. J. Chem. Soc., Chem. Commun. 1976, No. 3, 94-95. https://doi.org/10.1039/C39760000094.

182. Bloodworth, A. J.; Khan, J. A. J. Chem. Soc., Perkin Trans. 11980, 0 (0), 2450-2457. https://doi.org/10.1039/P19800002450.

183. Bloodworth, A. J.; Loveitt, M. E. J. Chem. Soc., Perkin Trans. 1 1978, 0 (6), 522-530. https://doi.org/10.1039/P19780000522.

184. Bloodworth, A. J.; Bothwell, B. D.; Collins, A. N.; Maidwell, N. L. Tetrahedron Lett. 1996, 37 (11), 1885-1888. https://doi.org/10.1016/0040-4039(96)00143-8. 
185. Bloodworth, A. J.; Korkodilos, D. Tetrahedron Lett. 1991, 32 (47), 6953-6956. https://doi.org/10.1016/0040-4039(91)80453-D.

186. Bloodworth, A. J.; Curtis, R. J. J. Chem. Soc., Chem. Commun. 1989, No. 3, 173-175. https://doi.org/10.1039/C39890000173.

187. Bloodworth, A. J.; Tallant, N. A. Tetrahedron Lett. 1990, 31 (48), 7077-7080. https://doi.org/10.1016/S0040-4039(00)97247-2.

188. Kishali, N. (Horasan); Sahin, E.; Kara, Y. Org. Lett. 2006, 8 (9), 1791-1793. https://doi.org/10.1021/ol060272s.

189. Krabbe, S. W.; Do, D. T.; Johnson, J. S. Org. Lett. 2012, 14 (23), 5932-5935. https://doi.org/10.1021/ol302848m.

190. Das, P.; Hasan, M. H.; Mitra, D.; Bollavarapu, R.; Valente, E. J.; Tandon, R.; Raucher, D.; Hamme, A. T. J. Org. Chem. 2019, 84 (11), 6992-7006. https://doi.org/10.1021/acs.joc.9b00746.

191. Harris, J. R.; Waetzig, S. R.; Woerpel, K. A. Org. Lett. 2009, 11 (15), 3290-3293. https://doi.org/10.1021/01901046z.

192. Weitz, E.; Scheffer, A. Chem. Ber. 1921, 54 (9), 2327-2344. https://doi.org/10.1002/cber.19210540922.

193. Murakami, N.; Kawanishi, M.; Itagaki, S.; Horii, T.; Kobayashi, M. Tetrahedron Lett. 2001, 42 (41), 7281-7285. https://doi.org/10.1016/S0040-4039(01)01493-9.

194. Murakami, N.; Kawanishi, M.; Itagaki, S.; Horii, T.; Kobayashi, M. Bioorg. Med. Chem. Lett. 2002, 12 (1), 69-72. https://doi.org/10.1016/S0960-894X(01)00673-4.

195. Kawanishi, M.; Kotoku, N.; Itagaki, S.; Horii, T.; Kobayashi, M. Bioorg. Med. Chem. Lett. 2004, 12 (20), 5297-5307. https://doi.org/10.1016/j.bmc.2004.04.051.

196. Yaremenko, I. A.; Vil', V. A.; Demchuk, D. V.; Terent'ev, A. O. Beilstein J. Org. Chem. 2016, 12 (1), 1647-1748. https://doi.org/10.3762/bjoc.12.162.

197. Xu, C.; Raible, J. M.; Dussault, P. H. Org. Lett. 2005, 7 (12), 2509-2511. https://doi.org/10.1021/ol050291m.

198. Jin, H.-X.; Liu, H.-H.; Zhang, Q.; Wu, Y. Tetrahedron Lett. 2005, 46 (34), 5767-5769. https://doi.org/10.1016/j.tetlet.2005.06.110.

199. Jin, H.-X.; Zhang, Q.; Kim, H.-S.; Wataya, Y.; Liu, H.-H.; Wu, Y. Tetrahedron 2006, 62 (33), 76997711. https://doi.org/10.1016/j.tet.2006.05.065.

200. Li, Y.; Zhang, Q.; Wittlin, S.; Jin, H.-X.; Wu, Y. Tetrahedron 2009, 65 (34), 6972-6985. https://doi.org/10.1016/.tet.2009.06.050.

201. Guerra, F. M.; Zubía, E.; Ortega, M. J.; Moreno-Dorado, F. J.; Massanet, G. M. Tetrahedron 2010, 66 (1), 157-163. https://doi.org/10.1016/j.tet.2009.11.026.

202. Barnych, B.; Vatèle, J.-M. Org. Lett. 2012, 14 (2), 564-567. https://doi.org/10.1021/ol203185f.

203. Silva, E. M. P.; Pye, R. J.; Brown, G. D.; Harwood, L. M. Eur. J. Org. Chem. 2012, 2012 (6), 1209-1216. https://doi.org/10.1002/ejoc.201101477.

204. Reisinger, C. M.; Wang, X.; List, B. Angew. Chem. Int. Ed. 2008, 47 (42), 8112-8115. https://doi.org/10.1002/anie.200803238.

205. Lifchits, O.; Mahlau, M.; Reisinger, C. M.; Lee, A.; Farès, C.; Polyak, I.; Gopakumar, G.; Thiel, W.; List, B. J. Am. Chem. Soc. 2013, 135 (17), 6677-6693. https://doi.org/10.1021/ja402058v.

206. Milas, N. A.; Mageli, O. L.; Golubovic, Aleksandar.; Arndt, R. W.; Ho, J. C. J. J. Am. Chem. Soc. 1963, 85 (2), 222-226. https://doi.org/10.1021/ja00885a022.

207. Rieche, A.; Bischoff, C.; Pulz, M. Chem. Ber. 1962, 95 (8), 2005-2008. https://doi.org/10.1002/cber.19620950822.

208. Howarth, J.; Wilson, D. Bioorg. Med. Chem. Lett. 2003, 13 (12), 2013-2015. https://doi.org/10.1016/S0960-894X(03)00326-3. 
209. Zdvizhkov, A. T.; Terent'ev, A. O.; Radulov, P. S.; Novikov, R. A.; Tafeenko, V. A.; Chernyshev, V. V.; Ilovaisky, A. I.; Levitsky, D. O.; Fleury, F.; Nikishin, G. I. Tetrahedron Lett. 2016, 57 (8), 949952. https://doi.org/10.1016/j.tetlet.2016.01.061.

210. Kandur, W. V.; Richert, K. J.; Rieder, C. J.; Thomas, A. M.; Hu, C.; Ziller, J. W.; Woerpel, K. A. Org. Lett. 2014, 16 (10), 2650-2653. https://doi.org/10.1021/ol500835f.

211. Gemma, S.; Kunjir, S.; Sanna Coccone, S.; Brindisi, M.; Moretti, V.; Brogi, S.; Novellino, E.; Basilico, N.; Parapini, S.; Taramelli, D.; Campiani, G.; Butini, S. J. Med. Chem. 2011, 54 (16), 5949-5953. https://doi.org/10.1021/jm200686d.

212. Gemma, S.; di Cerbo, L.; Relitti, N.; Vallone, A.; Brindisi, M.; Brogi, S.; Chemi, G.; Novellino, E.; Campiani, G.; Butini, S. Tetrahedron Letters 2018, 59 (49), 4330-4333. https://doi.org/10.1016/j.tetlet.2018.10.059.

213. Gemma, S.; Kunjir, S.; Brindisi, M.; Novellino, E.; Campiani, G.; Butini, S. Tetrahedron Letters 2013, 54 (10), 1233-1235. https://doi.org/10.1016/j.tetlet.2012.12.075.

214. D’Alessandro, S.; Alfano, G.; Di Cerbo, L.; Brogi, S.; Chemi, G.; Relitti, N.; Brindisi, M.; Lamponi, S.; Novellino, E.; Campiani, G.; Gemma, S.; Basilico, N.; Taramelli, D.; Baratto, M. C.; Pogni, R.; Butini, S. Bioorganic Chemistry 2019, 89, 103020. https://doi.org/10.1016/j.bioorg.2019.103020.

215. Snider, B. B.; Shi, Z. J. Am. Chem. Soc. 1992, 114 (5), 1790-1800. https://doi.org/10.1021/ja00031a038.

216. Dussault, P. H.; Eary, C. T.; Woller, K. R. J. Org. Chem. 1999, 64 (6), 1789-1797. https://doi.org/10.1021/j0981128q.

217. Snider, B. B.; O'Neil, S. V. Synth. Commun. 1995, 25 (7), 1085-1091. https://doi.org/10.1080/00397919508012671.

218. Wu, J.; Kadonaga, Y.; Hong, B.; Wang, J.; Lei, X. Angew. Chem. Int.Ed. 2019, 58 (32), 1087910883. https://doi.org/10.1002/anie.201903682.

219. Dussault, P. H.; Zope, U. R. Tetrahedron Lett. 1995, 36 (13), 2187-2190. https://doi.org/10.1016/0040-4039(95)00231-Z.

220. Windaus, A.; Brunken, J. Liebigs. Ann. 1928, 460 (1), 225-235. https://doi.org/10.1002/ilac. 19284600113.

221. Harwood, L. M.; Robertson, J.; Swallow, S. Synlett 1999, 1999 (09), 1359-1362. https://doi.org/10.1055/s-1999-2851.

222. Yao, G.; Steliou, K. Org. Lett. 2002, 4 (4), 485-488. https://doi.org/10.1021/ol016943y.

223. Jung, M.; Ham, J.; Song, J. Org. Lett. 2002, 4 (16), 2763-2765. https://doi.org/10.1021/ol026285x.

224. Sun, L.; Shah, F.; Helal, M. A.; Wu, Y.; Pedduri, Y.; Chittiboyina, A. G.; Gut, J.; Rosenthal, P. J.; Avery, M. A. J. Med. Chem. 2010, 53 (21), 7864-7868. https://doi.org/10.1021/im1006462.

225. Shen, X.-Y.; Peng, X.-S.; Wong, H. N. C. Org. Lett. 2016, 18 (5), 1032-1035. https://doi.org/10.1021/acs.orglett.6b00161.

226. Nicolaou, K. C.; Lu, M.; Totokotsopoulos, S.; Heretsch, P.; Giguère, D.; Sun, Y.-P.; Sarlah, D.; Nguyen, T. H.; Wolf, I. C.; Smee, D. F.; Day, C. W.; Bopp, S.; Winzeler, E. A. J. Am. Chem. Soc. 2012, 134 (41), 17320-17332. https://doi.org/10.1021/ja308429f.

227. Nicolaou, K. C.; Totokotsopoulos, S.; Giguère, D.; Sun, Y.-P.; Sarlah, D. J. Am. Chem. Soc. 2011, 133 (21), 8150-8153. https://doi.org/10.1021/ja2032635.

228. Liu, Y.-T.; Li, L.-P.; Xie, J.-H.; Zhou, Q.-L. Angew. Chem. Int. Ed. 2017, 56 (41), 12708-12711. https://doi.org/10.1002/anie.201706994.

229. Lee, R. J.; Lindley, M. R.; Pritchard, G. J.; Kimber, M. C. Chem. Commun. 2017, 53 (47), 63276330. https://doi.org/10.1039/C7CC03229C.

230. Mori, N.; Sakoda, D.; Watanabe, H. Tetrahedron Lett. 2017, 58 (40), 3884-3886. https://doi.org/10.1016/i.tetlet.2017.08.064. 
231. Cabrera-Afonso, M. J.; Lucena, S. R.; Juarranz, Á.; Urbano, A.; Carreño, M. C. Org. Lett. 2018, 20 (19), 6094-6098. https://doi.org/10.1021/acs.orglett.8b02515.

232. Yoshida, M.; Miura, M.; Nojima, M.; Kusabayashi, S. J. Am. Chem. Soc. 1983, 105 (20), 62796285. https://doi.org/10.1021/ja00358a016.

233. Miura, M.; Yoshida, M.; Nojima, M.; Kusabayashi, S. J. Chem. Soc., Chem. Commun. 1982, No. 7, 397-398. https://doi.org/10.1039/C39820000397.

234. Dussault, P. H.; Lee, H.-J.; Liu, X. J. Chem. Soc., Perkin Trans. 1 2000, 0 (17), 3006-3013. https://doi.org/10.1039/B001391I.

235. Dussault, P. H.; Liu, X. Tetrahedron Lett. 1999, 40 (36), 6553-6556. https://doi.org/10.1016/S0040-4039(99)01399-4.

236. Dussault, P. H.; Zope, U. Tetrahedron Lett. 1995, 36 (21), 3655-3658. https://doi.org/10.1016/0040-4039(95)00622-J.

237. Dussault, P. H.; Lee, I. Q.; Lee, H.-J.; Niu, Q. J.; Schultz, J. A.; Zope, U. R. J. Org. Chem. 2000, 65 (25), 8407-8414. https://doi.org/10.1021/j0991714z.

238. Ramirez, A.; Woerpel, K. A. Org. Lett. 2005, 7 (21), 4617-4620. https://doi.org/10.1021/ol051703u.

239. Hurlocker, B.; Miner, M. R.; Woerpel, K. A. Org. Lett. 2014, 16 (16), 4280-4283. https://doi.org/10.1021/ol5020015.

240. Xu, Z.-J.; Wittlin, S.; Wu, Y. Chem. Eur. J. 2017, 23 (9), 2031-2034. https://doi.org/10.1002/chem.201605871.

241. Pinet, A.; Nguyen, T. L.; Bernadat, G.; Figadère, B.; Ferrié, L. Org. Lett. 2019, 21 (12), 4729 4733. https://doi.org/10.1021/acs.orglett.9b01616.

242. Dussault, P. H.; Lee, H.-J.; Niu, Q. J. J. Org. Chem. 1995, 60 (4), 784-785. https://doi.org/10.1021/j000109a001.

243. Wang, X.; Dong, Y.; Wittlin, S.; Charman, S. A.; Chiu, F. C. K.; Chollet, J.; Katneni, K.; Mannila, J.; Morizzi, J.; Ryan, E.; Scheurer, C.; Steuten, J.; Santo Tomas, J.; Snyder, C.; Vennerstrom, J. L. J. Med. Chem. 2013, 56 (6), 2547-2555. https://doi.org/10.1021/jm400004u.

244. Zhao, Q.; Vargas, M.; Dong, Y.; Zhou, L.; Wang, X.; Sriraghavan, K.; Keiser, J.; Vennerstrom, J. L. J. Med. Chem. 2010, 53 (10), 4223-4233. https://doi.org/10.1021/jm100226t.

245. Wang, X.; Dong, Y.; Wittlin, S.; Creek, D.; Chollet, J.; Charman, S. A.; Santo Tomas, J.; Scheurer, C.; Snyder, C.; Vennerstrom, J. L. J. Med. Chem. 2007, 50 (23), 5840-5847. https://doi.org/10.1021/im0707673.

246. Martyn, D. C.; Ramirez, A. P.; Beattie, M. J.; Cortese, J. F.; Patel, V.; Rush, M. A.; Woerpel, K. A.; Clardy, J. Bioorganic \& Medicinal Chem. Lett. 2008, 18 (24), 6521-6524. https://doi.org/10.1016/j.bmcl.2008.10.083.

247. Gaschler, M. M.; Andia, A. A.; Liu, H.; Csuka, J. M.; Hurlocker, B.; Vaiana, C. A.; Heindel, D. W.; Zuckerman, D. S.; Bos, P. H.; Reznik, E.; Ye, L. F.; Tyurina, Y. Y.; Lin, A. J.; Shchepinov, M. S.; Chan, A. Y.; Peguero-Pereira, E.; Fomich, M. A.; Daniels, J. D.; Bekish, A. V.; Shmanai, V. V.; Kagan, V. E.; Mahal, L. K.; Woerpel, K. A.; Stockwell, B. R. Nat. Chem. Biol. 2018, 14 (5), 507515. https://doi.org/10.1038/s41589-018-0031-6.

248. Abrams, R. P.; Carroll, W. L.; Woerpel, K. A. ACS Chem. Biol. 2016, 11 (5), 1305-1312. https://doi.org/10.1021/acschembio.5b00900.

249. Dussault, P. H.; Liu, X. Org. Lett. 1999, 1 (9), 1391-1393. https://doi.org/10.1021/ol990954y.

250. Dussault, P. H.; K. Trullinger, T.; Cho-Shultz, S. Tetrahedron 2000, 56 (47), 9213-9220. https://doi.org/10.1016/S0040-4020(00)00894-2.

251. Pinet, A.; Figadère, B.; Ferrié, L. Adv. Synth. Cat. 2020, 362 (5), 1190-1194. https://doi.org/10.1002/adsc.201901145. 